\title{
Rh-Catalyzed Conjugate Addition of Arylzinc Chlorides to Thiochromones: A Highly Enantioselective Pathway for Accessing Chiral Thioflavanones
}

\author{
Ling Meng, Ming Yu Jin and Jun Wang* \\ Department of Chemistry, South University of Science and Technology of China, Shenzhen 518055, China
}

\section{Table of Contents}

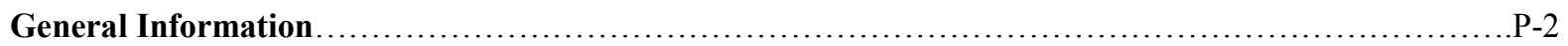

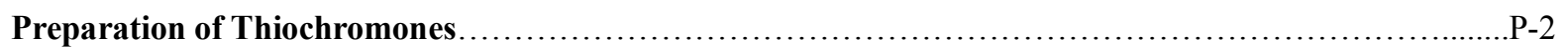

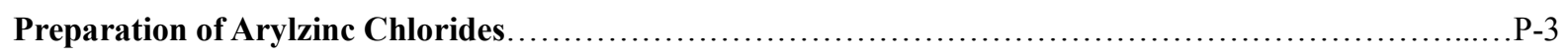

General Procedure for Addition of Arylzinc Chlorides to Thichromones...............................

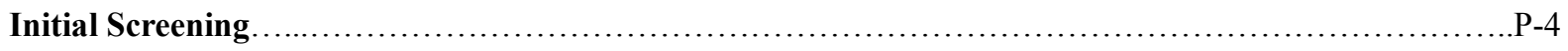

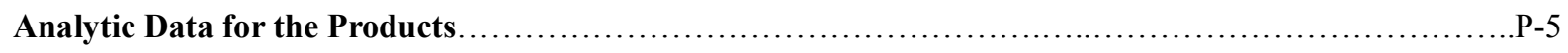

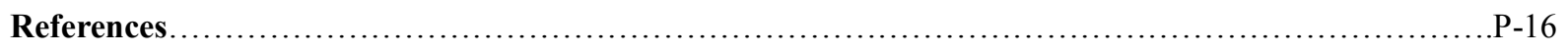

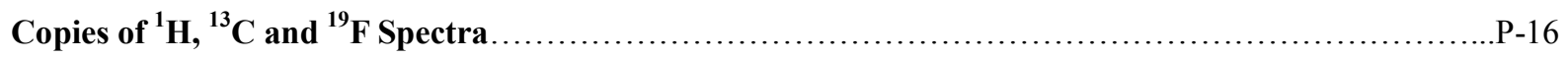

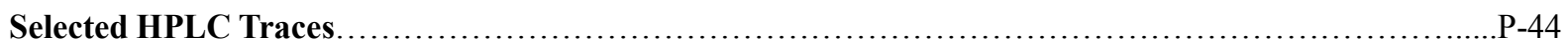




\section{General Information}

NMR Spectra were recorded on a Bruker DPX-400 spectrometer at $400 \mathrm{MHz}$ or $500 \mathrm{MHz}$ for ${ }^{1} \mathrm{H}$ NMR, $376 \mathrm{MHz}$ for ${ }^{19} \mathrm{~F} \mathrm{NMR}$ and $100 \mathrm{MHz}$ or $125 \mathrm{MHz}$ for ${ }^{13} \mathrm{C} \mathrm{NMR}$ in $\mathrm{CDCl}_{3}$ with tetramethylsilane (TMS) as internal standard, and ${ }^{19} \mathrm{~F}$ NMR chemical shifts were determined relative to $\mathrm{CFCl}_{3}$ as inter standard. Chemical shifts $(\delta)$ are reported in ppm, and coupling constants $(J)$ are in Hertz $(\mathrm{Hz})$. The following abbreviations were used to explain the multiplicities: $\mathrm{s}=$ singlet, $\mathrm{d}=$ doublet, $\mathrm{t}=$ triplet, $\mathrm{q}=$ quartet, $\mathrm{m}=$ multiplet, $\mathrm{br}=$ broad. Flash column chromatograph was carried out using 200-300 mesh silica gel at medium pressure or ODS-A-HG C18 reversed silica gel. High resolution mass spectra (HRMS) were recorded on a LC-TOF spectrometer. ESI-HRMS data were acquired using a Thermo LTQ Orbitrap XL Instrument equipped with an ESI source. Optical rotation was obtained on a Rudolph Research Analytical (Atopol I). HPLC analysis was performed on Agilent 1260 series, UV detection monitored at $254 \mathrm{~nm}$, using a Chiralcel OJ-3 column with hexane and $i$-PrOH as the eluent. All chemicals were purchased from Acros, Alfa Aesar and TCI, and used as received.

All air- and moisture-sensitive manipulations were carried out with standard Schlenk techniques under nitrogen or in a glove box under argon. All the liquid reagents were distilled under Ar.

\section{Preparation of Thiochromones ${ }^{1}$}

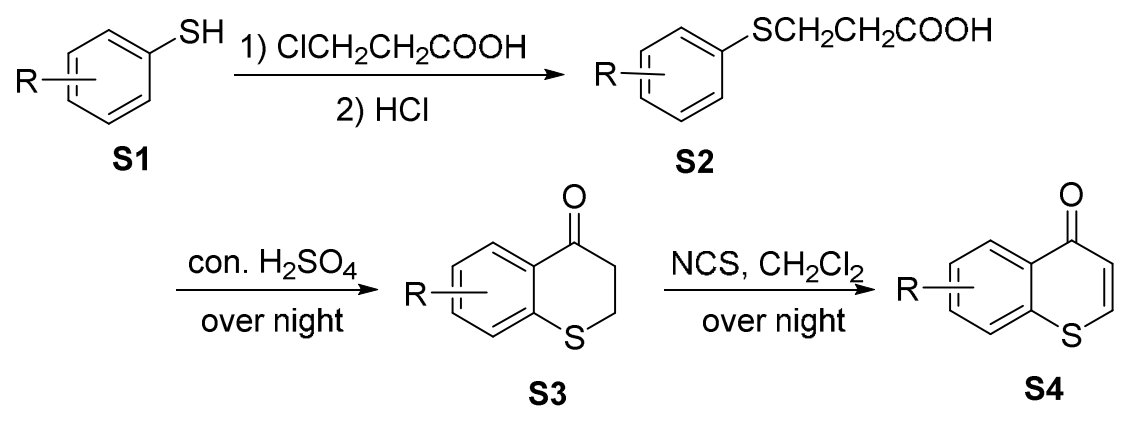

Thiochromone were prepared according to previously reported procedures. Thiophenols were purchased and used as received. A $250 \mathrm{~mL}$ flask was fitted with a stirrer bar and charged with $1 \mathrm{M}$ $\mathrm{NaOH}(50 \mathrm{~mL})$ and $1 \mathrm{M} \mathrm{Na}_{2} \mathrm{CO}_{3}(50 \mathrm{~mL})$. A solution of thiophenols $\mathbf{S 1}(100 \mathrm{mmol})$ in $60 \mathrm{~mL} \mathrm{EtOH}$ and 3-chloropropanoic acid (11 g, $102 \mathrm{mmol})$ in $40 \mathrm{~mL} \mathrm{H}_{2} \mathrm{O}$ were added respectively to the above solution. The reaction mixture was stirred at room temperature for $2 \mathrm{~h}$, and then temperature increased to reflux. After the reaction was completed as determined by TLC and cooled down to 
room temperature, EtOH was evaporated and then the aqueous phase was acidified to $\mathrm{pH} 1 \sim 2$ with conc. $\mathrm{HCl}$. The solution was extracted with $\mathrm{CH}_{2} \mathrm{Cl}_{2}$ for three times and the combined organic layers were dried over anhydrous $\mathrm{Na}_{2} \mathrm{SO}_{4}$, filtered, and concentrated. The residue was purified by flash column chromatography (hexane/EtOAc, 20/1 to 3/1) to produce 3-(phenylthio)propanoic acid $\mathbf{S 2}$ in $85-95 \%$ yield.

A $250 \mathrm{~mL}$ flask was filled with $45 \mathrm{~mL}$ conc. $\mathrm{H}_{2} \mathrm{SO}_{4}$ and cooled down to $0{ }^{\circ} \mathrm{C}$. $\mathbf{S 2}$ was added slowly. Then the mixture was stirred at room temperature overnight. After the reaction was completed as determined by TLC, the reaction was quenched by pouring onto ice and extracted with $\mathrm{CH}_{2} \mathrm{Cl}_{2}$ for three times. The combined organic layers were dried over anhydrous $\mathrm{Na}_{2} \mathrm{SO}_{4}$, filtered, and concentrated. The residue was purified by flash column chromatography (hexane/EtOAc, 10/1) to afford thiochroman-4-one $\mathbf{S 3}$ in 70-95\% yield.

To a solution of $\mathbf{S 3}(30.5 \mathrm{mmol})$ in $\mathrm{CH}_{2} \mathrm{Cl}_{2}(100 \mathrm{ml})$ was added $\mathrm{N}$-chlorosuccinimide (NCS) (30.5 mmol, 1.0 equive) in one portion. This was stirred for $20 \mathrm{~min}$ at $5{ }^{\circ} \mathrm{C}$, and then allowed to warm to room temprature overnight. Water $(50 \mathrm{ml})$ was added and the organic layer was separated and dried $\left(\mathrm{Na}_{2} \mathrm{SO}_{4}\right)$, and the product purified by column chromatography (hexane/EtOAc, 5/1) to give thiochromone $\mathbf{S 4}$ in $40-80 \%$ yield.

\section{Preparation of Arylzinc Chlorides ${ }^{2}$}

$\mathrm{ZnCl}_{2}$ (Aldrich; 99.999\% trace metals basis; $2.73 \mathrm{~g}, 20.0 \mathrm{mmol}$ ) was added quickly to an oven-dried $50 \mathrm{~mL}$ vial equipped with a stir bar. THF (distilled before using; $20 \mathrm{~mL}$ ) was added to this vial, and the resulting mixture was stirred vigorously until the $\mathrm{ZnCl}_{2}$ had completely dissolved. A solution of $n$-BuLi (2.4 M in THF; $4.6 \mathrm{~mL}, 11.0 \mathrm{mmol}$; 1.1 equiv) was added via a syringe over $5 \mathrm{~min}$ to the vial that contained the solution of the aryl bromide (distilled before the reaction; $1.1 \mathrm{~mL}, 10 \mathrm{mmol}, 1.0$ equiv), which had been cooled to $-78^{\circ} \mathrm{C}$. The mixture was allowed to stir at $-78^{\circ} \mathrm{C}$ for $1 \mathrm{~h}$, and then the solution of $\mathrm{ZnCl}_{2}(0.3 \mathrm{M} ; 15.00 \mathrm{~mL}, 15 \mathrm{mmol} ; 1.50$ equiv) was added to the vial. The mixture was allowed to warm to r.t. and stirred for $1.5 \mathrm{~h}$ at $\mathrm{rt}$, affording a solution of arylzinc chrorides in THF (0.3 M). 


\section{General Procedure for Addition of Arylzinc Chlorides to Thichromones}

An oven-dried vial fitted with a stirrer bar was charged with $1.25 \mathrm{~mol} \%[\mathrm{Rh}(\mathrm{COD}) \mathrm{Cl}]_{2}(0.9 \mathrm{mg}, \mathrm{Rh})$ and $2.75 \mathrm{~mol} \%(R)-3,4,5-\mathrm{MeO}-\mathrm{BIPHEP}(3.8 \mathrm{mg}, 1.1$ equiv) in THF $(1.0 \mathrm{~mL})$ and the mixture was stirred at room temperature for $30 \mathrm{~min}$. And then thiochromone $1 \mathrm{a}(0.15 \mathrm{mmol})$ was added and stirred for another $10 \mathrm{~min}$. ArZnCl $(1.0 \mathrm{~mL}, 0.3 \mathrm{mmol} ; 0.3 \mathrm{M}$ solution in $\mathrm{THF})$ and chlorotrimethylsilane $(77 \mu \mathrm{L}, 0.45 \mathrm{mmol})$ were simultaneously added dropwise to the vial over 10 min and the resulting mixture was stirred at room temperature until the reaction completed. The reaction was quenched with $\mathrm{HCl}(10 \%$ aqueous) and the mixture was stirred for $1 \mathrm{~h}$ at room temperature. This was extracted with EtOAc and the organic layer was dried over $\mathrm{Na}_{2} \mathrm{SO}_{4}$, filtered, and concentrated under vacuum. The residue was chromatographed on silica gel (EtOAc/PE, 1:80) to get the desired products.

\section{Initial Screening}

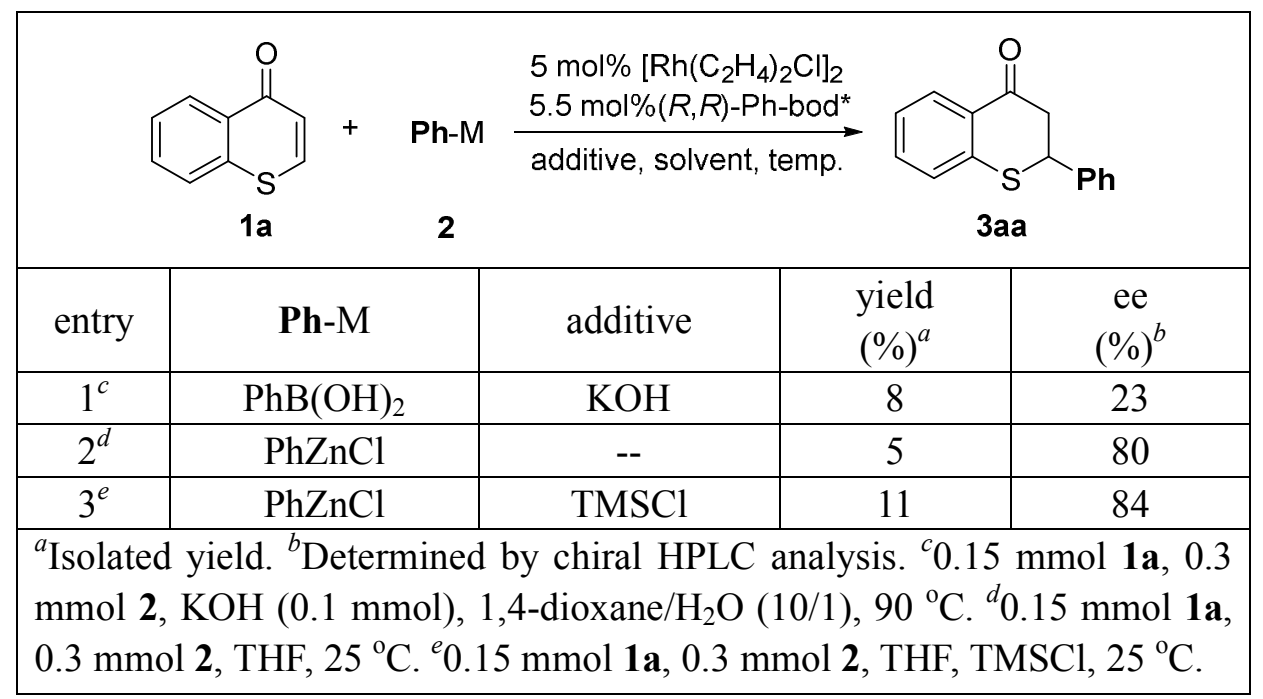




\section{Analytic Data for the Products}

\section{2-phenylthiochroman-4-one (3aa)}<smiles>O=C1CC(c2ccccc2)Sc2ccccc21</smiles>

Light yellow solid $\left(32.6 \mathrm{mg}, 91 \%\right.$ yield); $\mathrm{mp} 56-58{ }^{\circ} \mathrm{C}$; $[\alpha]_{\mathrm{D}}^{25}=-144.6\left(c \quad 1.00, \mathrm{CHCl}_{3}\right)$; ee was determined to be $97 \%$ by HPLC analysis with a Chiralcel OJ-3 column (hexane/2-propanol 60:40, $1.0 \mathrm{~mL} / \mathrm{min}, 254 \mathrm{~nm}) ; \mathrm{t}_{\mathrm{r}}($ minor $)=11.3 \mathrm{~min}, \mathrm{t}_{\mathrm{r}}($ major $)=20.2 \mathrm{~min} ;{ }^{1} \mathrm{H} \mathrm{NMR}\left(400 \mathrm{MHz}, \mathrm{CDCl}_{3}\right) \delta$ $8.12(\mathrm{dd}, J=8.0,1.3 \mathrm{~Hz}, 1 \mathrm{H}), 7.40-7.28(\mathrm{~m}, 6 \mathrm{H}), 7.23-7.22(\mathrm{~m}, 1 \mathrm{H}), 7.17-7.13(\mathrm{~m}, 1 \mathrm{H}), 4.66(\mathrm{dd}, J$ $=13.0,2.9 \mathrm{~Hz}, 1 \mathrm{H}), 3.21(\mathrm{ddd}, J=19.5,16.4,8.1 \mathrm{~Hz}, 2 \mathrm{H}) .{ }^{13} \mathrm{C}\left\{{ }^{1} \mathrm{H}\right\} \mathrm{NMR}\left(100 \mathrm{MHz}, \mathrm{CDCl}_{3}\right) \delta$ $194.1,141.9,138.2,133.4,130.2,129.0,128.8,128.3,127.3,127.0,125.0,46.4$, 45.2; HRMS (ESI-ion trap) $m / z:[\mathrm{M}+\mathrm{H}]^{+}$calcd for $\mathrm{C}_{15} \mathrm{H}_{13} \mathrm{OS}, 241.0682$; found 241.0679.

\section{2-(4-methoxyphenyl)thiochroman-4-one (3ab)}

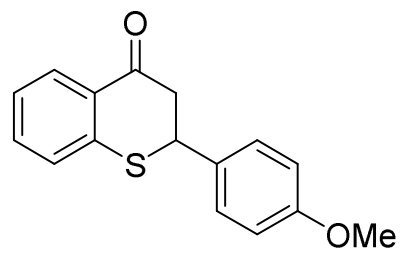

Light yellow solid $\left(34.1 \mathrm{mg}, 84 \%\right.$ yield); mp $72-74{ }^{\circ} \mathrm{C}$; $[\alpha]_{\mathrm{D}}^{25}=-114.9\left(c 1.33, \mathrm{CHCl}_{3}\right)$; ee was determined to be $85 \%$ by HPLC analysis with a Chiralcel OJ-3 column (hexane/2-propanol 60:40, $1.0 \mathrm{~mL} / \mathrm{min}, 254 \mathrm{~nm}) ; \mathrm{t}_{\mathrm{r}}($ minor $)=14.8 \mathrm{~min}, \mathrm{t}_{\mathrm{r}}($ major $)=19.9 \mathrm{~min} ;{ }^{1} \mathrm{H} \mathrm{NMR}\left(500 \mathrm{MHz}, \mathrm{CDCl}_{3}\right) \delta$ $8.14(\mathrm{dd}, J=8.0,1.4 \mathrm{~Hz}, 1 \mathrm{H}), 7.40(\mathrm{td}, J=8.0,1.5 \mathrm{~Hz}, 1 \mathrm{H}), 7.36-7.33(\mathrm{~m}, 2 \mathrm{H}), 7.27(\mathrm{~d}, J=8.7 \mathrm{~Hz}$, 1H), 7.21-7.18 (m, 1H), 6.92-6.89 (m, 2H), 4.67 (dd, $J=13.3,2.8 \mathrm{~Hz}, 1 \mathrm{H}), 3.81$ (s, 3H), 3.29 (dd, $J$ $=16.4,13.3 \mathrm{~Hz}, 1 \mathrm{H}), 3.17(\mathrm{dd}, J=16.4,2.9 \mathrm{~Hz}, 1 \mathrm{H}) .{ }^{13} \mathrm{C}\left\{{ }^{1} \mathrm{H}\right\} \mathrm{NMR}\left(125 \mathrm{MHz}, \mathrm{CDCl}_{3}\right) \delta 194.6$, $159.5,142.2,133.6,130.4,130.3,129.1,128.5,127.1,125.1,114.2,55.3,46.8,44.9$; HRMS (ESI-ion trap) $m / z$ : $[\mathrm{M}+\mathrm{H}]^{+}$calcd for $\mathrm{C}_{16} \mathrm{H}_{15} \mathrm{O}_{2} \mathrm{~S}, 271.0787$; found 271.0785.

\section{2-(4-methylphenyl)thiochroman-4-one (3ac)}




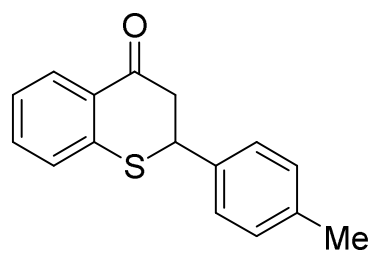

White solid (33.1 mg, 87\% yield); $\mathrm{mp} 52-54{ }^{\circ} \mathrm{C} ;[\alpha]_{\mathrm{D}}^{25}=-135.4\left(c 0.66, \mathrm{CHCl}_{3}\right)$; ee was determined to be $94 \%$ by HPLC analysis with a Chiralcel OJ-3 column (hexane/2-propanol 60:40, $1.0 \mathrm{~mL} / \mathrm{min}$, $254 \mathrm{~nm}) ; \mathrm{t}_{\mathrm{r}}($ minor $)=10.2 \mathrm{~min}, \mathrm{t}_{\mathrm{r}}($ major $)=14.0 \mathrm{~min} ;{ }^{1} \mathrm{H} \mathrm{NMR}\left(500 \mathrm{MHz}, \mathrm{CDCl}_{3}\right) \delta 8.15(\mathrm{~d}, J=7.9$ $\mathrm{Hz}, 1 \mathrm{H}), 7.41(\mathrm{t}, J=7.6 \mathrm{~Hz}, 1 \mathrm{H}), 7.30(\mathrm{dd}, J=17.1,7.9 \mathrm{~Hz}, 3 \mathrm{H}), 7.20$ (dd, $J=11.7,7.9 \mathrm{~Hz}, 3 \mathrm{H})$, $4.70(\mathrm{dd}, J=13.2,2.7 \mathrm{~Hz}, 1 \mathrm{H}), 3.31(\mathrm{dd}, J=16.4,13.3 \mathrm{~Hz}, 1 \mathrm{H}), 3.19(\mathrm{dd}, J=16.4,2.8 \mathrm{~Hz}, 1 \mathrm{H})$, $2.36(\mathrm{~s}, 3 \mathrm{H}) .{ }^{13} \mathrm{C}\left\{{ }^{1} \mathrm{H}\right\}$ NMR $\left(125 \mathrm{MHz}, \mathrm{CDCl}_{3}\right) \delta 194.6,142.2,138.3,135.4,133.6,130.4,129.6$, 129.2, 127.3, 127.2, 125.2, 46.8, 45.2, 21.1; HRMS (ESI-ion trap) $m / z:[\mathrm{M}+\mathrm{H}]^{+}$calcd for $\mathrm{C}_{16} \mathrm{H}_{15} \mathrm{OS}$, 255.0838; found 255.0831 .

\section{2-(3-methylphenyl)thiochroman-4-one (3ad)}<smiles>Cc1cccc(C2CC(=O)c3ccccc3S2)c1</smiles>

Viscous yellow liquid (28.0 mg, 74\% yield); $[\alpha]_{\mathrm{D}}^{25}=-98.8\left(c 0.50, \mathrm{CHCl}_{3}\right)$; ee was determined to be 95\% by HPLC analysis with a Chiralcel OJ-3 column (hexane/2-propanol 60:40, $1.0 \mathrm{~mL} / \mathrm{min}, 254$ $\mathrm{nm}) ; \mathrm{t}_{\mathrm{r}}($ minor $)=11.2 \mathrm{~min}, \mathrm{t}_{\mathrm{r}}($ major $)=22.5 \mathrm{~min} ;{ }^{1} \mathrm{H} \mathrm{NMR}\left(400 \mathrm{MHz}, \mathrm{CDCl}_{3}\right) \delta 8.15(\mathrm{dd}, J=8.0$, $1.4 \mathrm{~Hz}, 1 \mathrm{H}), 7.43-7.39(\mathrm{~m}, 1 \mathrm{H}), 7.29-7.14(\mathrm{~m}, 6 \mathrm{H}), 4.68(\mathrm{dd}, J=13.2,3.0 \mathrm{~Hz}, 1 \mathrm{H}), 3.31(\mathrm{dd}, J=$ 16.4, $13.2 \mathrm{~Hz}, 1 \mathrm{H}), 3.19(\mathrm{dd}, J=16.4,3.0 \mathrm{~Hz}, 1 \mathrm{H}), 2.37(\mathrm{~s}, 3 \mathrm{H}) .{ }^{13} \mathrm{C}\left\{{ }^{1} \mathrm{H}\right\} \mathrm{NMR}\left(100 \mathrm{MHz}, \mathrm{CDCl}_{3}\right) \delta$ $194.5,142.2,138.7,138.3,133.6,130.4,129.2,129.2,128.8,128.1,127.2,125.2,124.4,46.7,45.5$, 21.4; HRMS (ESI-ion trap) $m / z$ : [M+H $]^{+}$calcd for $\mathrm{C}_{16} \mathrm{H}_{15} \mathrm{OS}, 255.0838$; found 255.0831.

\section{2-(2-methylphenyl)thiochroman-4-one (3ae)}<smiles>Cc1ccccc1C1CC(=O)c2ccccc2S1</smiles> 
White solid (23.0 mg, $60 \%$ yield); $\mathrm{mp} 80-82{ }^{\circ} \mathrm{C} ;[\alpha]_{\mathrm{D}}^{25}=-6.9\left(c 1.33, \mathrm{CHCl}_{3}\right)$; ee was determined to be 7\% by HPLC analysis with a Chiralcel OJ-3 column (hexane/2-propanol 60:40, $1.0 \mathrm{~mL} / \mathrm{min}, 254$ $\mathrm{nm}) ; \mathrm{t}_{\mathrm{r}}($ minor $)=10.1 \mathrm{~min}, \mathrm{t}_{\mathrm{r}}$ (major) $=11.6 \mathrm{~min} ;{ }^{1} \mathrm{H} \mathrm{NMR}\left(500 \mathrm{MHz}, \mathrm{CDCl}_{3}\right) \delta 8.17(\mathrm{dd}, J=8.0$, $1.4 \mathrm{~Hz}, 1 \mathrm{H}), 7.49-7.47(\mathrm{~m}, 1 \mathrm{H}), 7.42(\mathrm{td}, J=8.0,1.5 \mathrm{~Hz}, 1 \mathrm{H}), 7.25(\mathrm{ddt}, J=16.3,15.0,8.0 \mathrm{~Hz}, 5 \mathrm{H})$, $4.92(\mathrm{dd}, J=13.6,2.5 \mathrm{~Hz}, 1 \mathrm{H}), 3.38$ (dd, $J=16.4,13.6 \mathrm{~Hz}, 1 \mathrm{H}), 3.14$ (dd, $J=16.4,2.5 \mathrm{~Hz}, 1 \mathrm{H})$, 2.43 (s, 3H). ${ }^{13} \mathrm{C}\left\{{ }^{1} \mathrm{H}\right\}$ NMR (125 MHz, $\left.\mathrm{CDCl}_{3}\right) \delta 194.9,142.4,136.2,136.1,133.6,131.0,130.3$, 129.3, 128.2, 127.3, 126.7, 126.5, 125.2, 46.2, 41.6, 19.2; HRMS (ESI-ion trap) $\mathrm{m} / \mathrm{z}:[\mathrm{M}+\mathrm{H}]^{+}$calcd for $\mathrm{C}_{16} \mathrm{H}_{15} \mathrm{OS}, 255.0838$; found 255.0834 .

\section{2-(4-fluorophenyl)thiochroman-4-one (3af)}<smiles>O=C1CC(c2ccc(F)cc2)Sc2ccccc21</smiles>

Yellow solid (18.2 mg, 47\% yield); $\mathrm{mp} 83-85{ }^{\circ} \mathrm{C} ;[\alpha]_{\mathrm{D}}^{25}=-70.4\left(\right.$ c $\left.0.12, \mathrm{CHCl}_{3}\right)$; ee was determined to be $91 \%$ by HPLC analysis with a Chiralcel OJ-3 column (hexane/2-propanol 60:40, $1.0 \mathrm{~mL} / \mathrm{min}$, $254 \mathrm{~nm}) ; \mathrm{t}_{\mathrm{r}}($ minor $)=9.2 \mathrm{~min}, \mathrm{t}_{\mathrm{r}}($ major $)=11.1 \mathrm{~min} ;{ }^{1} \mathrm{H} \mathrm{NMR}\left(500 \mathrm{MHz}, \mathrm{CDCl}_{3}\right) \delta 8.15(\mathrm{~d}, J=7.9$ $\mathrm{Hz}, 1 \mathrm{H}), 7.42(\mathrm{dt}, J=8.3,6.7 \mathrm{~Hz}, 3 \mathrm{H}), 7.28(\mathrm{~d}, J=7.9 \mathrm{~Hz}, 1 \mathrm{H}), 7.22(\mathrm{t}, J=7.6 \mathrm{~Hz}, 1 \mathrm{H}), 7.08(\mathrm{t}, J=$ $8.3 \mathrm{~Hz}, 2 \mathrm{H}), 4.71(\mathrm{dd}, J=12.9,2.0 \mathrm{~Hz}, 1 \mathrm{H}), 3.32-3.26(\mathrm{~m}, 1 \mathrm{H}), 3.19$ (dd, $J=16.3,2.2 \mathrm{~Hz}, 1 \mathrm{H})$. ${ }^{13} \mathrm{C}\left\{{ }^{1} \mathrm{H}\right\} \mathrm{NMR}\left(125 \mathrm{MHz}, \mathrm{CDCl}_{3}\right) \delta 194.1,162.5\left(\mathrm{~d}, J_{\mathrm{F}-\mathrm{C}}=246.3 \mathrm{~Hz}\right), 141.8,134.2\left(\mathrm{~d}, J_{\mathrm{F}-\mathrm{C}}=3.75\right.$ $\mathrm{Hz}), 133.7,130.3,129.2\left(\mathrm{~d}, J_{\mathrm{F}-\mathrm{C}}=7.5 \mathrm{~Hz}\right), 129.1,127.2,125.3,115.9\left(\mathrm{~d}, J_{\mathrm{F}-\mathrm{C}}=21.3 \mathrm{~Hz}\right), 46.8,44.7$. ${ }^{19} \mathrm{~F}$ NMR (376 MHz, $\left.\mathrm{CDCl}_{3}\right) \delta$-113.07; HRMS (ESI-ion trap) $m / z$ : $[\mathrm{M}+\mathrm{H}]^{+}$calcd for $\mathrm{C}_{15} \mathrm{H}_{12} \mathrm{FOS}$, 259.0587; found 259.0584 .

\section{2-(4-bromophenyl)thiochroman-4-one (3ag)}<smiles>O=C1CC(c2ccc(Br)cc2)Sc2ccccc21</smiles>

Yellow solid (24.8 mg, 52\% yield); mp $128-130{ }^{\circ} \mathrm{C} ;[\alpha]_{\mathrm{D}}^{25}=-65.4$ (c $\left.0.92, \mathrm{CHCl}_{3}\right)$; ee was 
determined to be $95 \%$ by HPLC analysis with a Chiralcel OJ-3 column (hexane/2-propanol 60:40, $1.0 \mathrm{~mL} / \mathrm{min}, 254 \mathrm{~nm}) ; \mathrm{t}_{\mathrm{r}}$ (minor) $=9.5 \mathrm{~min}, \mathrm{t}_{\mathrm{r}}$ (major) $=11.3 \mathrm{~min} ;{ }^{1} \mathrm{H} \mathrm{NMR}\left(500 \mathrm{MHz}, \mathrm{CDCl}_{3}\right) \delta 8.14$ $(\mathrm{dd}, J=8.0,1.4 \mathrm{~Hz}, 1 \mathrm{H}), 7.52-7.49$ (m, 2H), 7.42 (td, $J=8.0,1.5 \mathrm{~Hz}, 1 \mathrm{H}), 7.32-7.27$ (m, 3H), 7.23$7.20(\mathrm{~m}, 1 \mathrm{H}), 4.67$ (dd, $J=12.7,3.1 \mathrm{~Hz}, 1 \mathrm{H}), 3.27$ (dd, $J=16.4,12.7 \mathrm{~Hz}, 1 \mathrm{H}), 3.18$ (dd, $J=16.4$, $3.2 \mathrm{~Hz}, 1 \mathrm{H}) .{ }^{13} \mathrm{C}\left\{{ }^{1} \mathrm{H}\right\}$ NMR $\left(125 \mathrm{MHz}, \mathrm{CDCl}_{3}\right) \delta 193.9,141.5,137.4,133.8,132.1,130.3,129.2$, 129.1, 127.2, 125.4, 122.4, 46.4, 44.8; HRMS (ESI-ion trap) $m / z$ : [M]-H] calcd for $\mathrm{C}_{15} \mathrm{H}_{10} \mathrm{BrOS}$, 316.9641 ; found 316.9644 .

\section{2-(4-(trifluoromethyl)phenyl)thiochroman-4-one (3ah)}

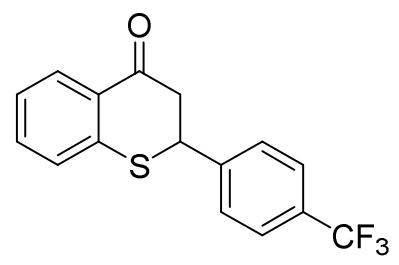

White solid (9.3 mg, 20\% yield); $\mathrm{mp} 88-90{ }^{\circ} \mathrm{C} ;[\alpha]_{\mathrm{D}}^{25}=-18.6\left(c 1.33, \mathrm{CHCl}_{3}\right)$; ee was determined to be $10 \%$ by HPLC analysis with a Chiralcel OJ-3 column (hexane/2-propanol 60:40, $1.0 \mathrm{~mL} / \mathrm{min}, 254$ $\mathrm{nm}) ; \mathrm{t}_{\mathrm{r}}($ minor $)=6.3 \mathrm{~min}, \mathrm{t}_{\mathrm{r}}($ major $)=7.3 \mathrm{~min} ;{ }^{1} \mathrm{H} \mathrm{NMR}\left(400 \mathrm{MHz}, \mathrm{CDCl}_{3}\right) \delta 8.15(\mathrm{dd}, J=7.9,1.3$ $\mathrm{Hz}, 1 \mathrm{H}), 7.65$ (d, $J=8.2 \mathrm{~Hz}, 2 \mathrm{H}), 7.56(\mathrm{~d}, J=8.4 \mathrm{~Hz}, 2 \mathrm{H}), 7.44$ (ddd, $J=8.0,7.3,1.6 \mathrm{~Hz}, 1 \mathrm{H})$, $7.30-7.21(\mathrm{~m}, 3 \mathrm{H}), 4.77(\mathrm{dd}, J=12.3,3.3 \mathrm{~Hz}, 1 \mathrm{H}), 3.27(\mathrm{ddd}, J=19.7,16.4,7.8 \mathrm{~Hz}, 2 \mathrm{H}) .{ }^{13} \mathrm{C}\left\{{ }^{1} \mathrm{H}\right\}$ NMR $\left(100 \mathrm{MHz} \mathrm{CDCl}_{3}\right) \delta 193.6,142.4,141.2,133.8,130.7$ (q, $\left.J_{\mathrm{F}-\mathrm{C}}=32.5 \mathrm{~Hz}\right), 130.3,129.3,127.9$, $127.2,125.9\left(\mathrm{q}, J_{\mathrm{F}-\mathrm{C}}=3.8 \mathrm{~Hz}\right), 125.5,124.0\left(\mathrm{q}, J_{\mathrm{F}-\mathrm{C}}=271.3 \mathrm{~Hz}\right), 46.3,44.9 .{ }^{19} \mathrm{~F} \mathrm{NMR}(376 \mathrm{MHz}$, $\left.\mathrm{CDCl}_{3}\right) \delta-62.70$; HRMS (ESI-ion trap) $\mathrm{m} / \mathrm{z}$ : [M]-H] calcd for $\mathrm{C}_{16} \mathrm{H}_{10} \mathrm{~F}_{3} \mathrm{OS}$, 307.0410; found 307.0412 .

\section{2-(3,5-dimethylphenyl)thiochroman-4-one (3ai)}<smiles>Cc1cc(C)cc(C2CC(=O)c3ccccc3S2)c1</smiles>

White solid (36.3 mg, 90\% yield); $\mathrm{mp} 74-76{ }^{\circ} \mathrm{C} ;[\alpha]_{\mathrm{D}}^{25}=-109.2\left(c 1.00, \mathrm{CHCl}_{3}\right)$; ee was determined 
to be $95 \%$ by HPLC analysis with a Chiralcel OJ-3 column (hexane/2-propanol 60:40, $1.0 \mathrm{~mL} / \mathrm{min}$, $254 \mathrm{~nm}) ; \mathrm{t}_{\mathrm{r}}($ minor $)=6.0 \mathrm{~min}, \mathrm{t}_{\mathrm{r}}($ major $)=8.8 \mathrm{~min} ;{ }^{1} \mathrm{H} \mathrm{NMR}\left(500 \mathrm{MHz}, \mathrm{CDCl}_{3}\right) \delta 8.14(\mathrm{dd}, J=8.0$, $1.4 \mathrm{~Hz}, 1 \mathrm{H}), 7.40$ (ddd, $J=8.0,7.3,1.5 \mathrm{~Hz}, 1 \mathrm{H}), 7.27$ (dd, $J=7.9,0.7 \mathrm{~Hz}, 1 \mathrm{H}), 7.20$ (td, $J=7.8,1.1$ Hz, 1H), 7.03 (s, 2H), 6.97 (s, 1H), 4.64 (dd, $J=13.5,2.8 \mathrm{~Hz}, 1 \mathrm{H}), 3.29$ (dd, $J=16.4,13.5 \mathrm{~Hz}, 1 \mathrm{H})$, $3.16(\mathrm{dd}, J=16.4,2.8 \mathrm{~Hz}, 1 \mathrm{H}), 2.32(\mathrm{~s}, 6 \mathrm{H}) .{ }^{13} \mathrm{C}\left\{{ }^{1} \mathrm{H}\right\} \mathrm{NMR}\left(125 \mathrm{MHz}, \mathrm{CDCl}_{3}\right) \delta 194.6,142.3,138.6$, 138.2, 133.6, 130.3, 130.1, 129.2, 127.2, 125.1, 125.1, 46.7, 45.5, 21.3; HRMS (ESI-ion trap) $m / z$ : $[\mathrm{M}+\mathrm{H}]^{+}$calcd for $\mathrm{C}_{17} \mathrm{H}_{17} \mathrm{OS}, 269.0995$; found 269.0989.

\section{2-(naphthalen-2-yl)thiochroman-4-one (3aj)}<smiles>O=C1CC(c2ccc3ccccc3c2)Sc2ccccc21</smiles>

White solid (37.0 mg, 85\% yield); mp $131-132{ }^{\circ} \mathrm{C}$; $[\alpha]_{\mathrm{D}}^{25}=-116.2\left(c\right.$ 1.05, $\left.\mathrm{CHCl}_{3}\right)$; ee was determined to be $95 \%$ by HPLC analysis with a Chiralcel OJ-3 column (hexane/2-propanol 60:40, $1.0 \mathrm{~mL} / \mathrm{min}, 254 \mathrm{~nm}) ; \mathrm{t}_{\mathrm{r}}($ minor $)=18.4 \mathrm{~min}, \mathrm{t}_{\mathrm{r}}($ major $)=25.1 \mathrm{~min} ;{ }^{1} \mathrm{H} \mathrm{NMR}\left(400 \mathrm{MHz}, \mathrm{CDCl}_{3}\right) \delta$ $8.17(\mathrm{dd}, J=7.9,1.1 \mathrm{~Hz}, 1 \mathrm{H}), 7.86-7.81(\mathrm{~m}, 4 \mathrm{H}), 7.54-7.48(\mathrm{~m}, 3 \mathrm{H}), 7.42-7.38(\mathrm{~m}, 1 \mathrm{H}), 7.28(\mathrm{~d}, J$ $=7.7 \mathrm{~Hz}, 1 \mathrm{H}), 7.21(\mathrm{dd}, J=13.9,5.9 \mathrm{~Hz}, 1 \mathrm{H}), 4.86(\mathrm{dd}, J=13.0,2.8 \mathrm{~Hz}, 1 \mathrm{H}), 3.42(\mathrm{dd}, J=16.4$, $13.0 \mathrm{~Hz}, 1 \mathrm{H}), 3.28(\mathrm{dd}, J=16.4,2.9 \mathrm{~Hz}, 1 \mathrm{H}) .{ }^{13} \mathrm{C}\left\{{ }^{1} \mathrm{H}\right\} \mathrm{NMR}\left(100 \mathrm{MHz}, \mathrm{CDCl}_{3}\right) \delta 194.3,141.9$, $135.7,133.6,133.2,133.1,130.4,129.2,128.8,128.0,127.7,127.2,126.5,126.5,126.4,125.2$, 125.1, 46.6, 45.6; HRMS (ESI-ion trap) $\mathrm{m} / \mathrm{z}$ : $[\mathrm{M}+\mathrm{H}]^{+}$calcd for $\mathrm{C}_{19} \mathrm{H}_{15} \mathrm{OS}$, 291.0838; found 291.0831 .

\section{2-(furan-2-yl)thiochroman-4-one (3ak)}

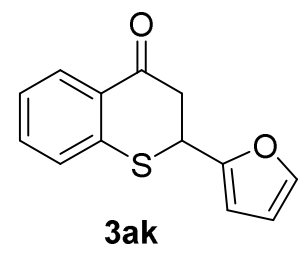

Light yellow solid $\left(22.1 \mathrm{mg}, 64 \%\right.$ yield); mp $63-65^{\circ} \mathrm{C} ;[\alpha]_{\mathrm{D}}^{25}=-3.0\left(c \quad 1.30, \mathrm{CHCl}_{3}\right)$; ee was determined to be $4 \%$ by HPLC analysis with a Chiralcel OJ-3 column (hexane/2-propanol 60:40, 1.0 
$\mathrm{mL} / \min , 254 \mathrm{~nm}) ; \mathrm{t}_{\mathrm{r}}($ major $)=9.5 \mathrm{~min}, \mathrm{t}_{\mathrm{r}}($ minor $)=12.2 \mathrm{~min} ;{ }^{1} \mathrm{H} \mathrm{NMR}\left(500 \mathrm{MHz}, \mathrm{CDCl}_{3}\right) \delta 8.13$ $(\mathrm{dd}, J=8.0,1.4 \mathrm{~Hz}, 1 \mathrm{H}), 7.42-7.37(\mathrm{~m}, 2 \mathrm{H}), 7.26(\mathrm{dd}, J=7.9,0.8 \mathrm{~Hz}, 1 \mathrm{H}), 7.22-7.18(\mathrm{~m}, 1 \mathrm{H}), 6.30$ (dd, $J=3.3,1.9 \mathrm{~Hz}, 1 \mathrm{H}), 6.25$ (d, $J=3.3 \mathrm{~Hz}, 1 \mathrm{H}), 4.75$ (dd, $J=8.4,4.8 \mathrm{~Hz}, 1 \mathrm{H}), 3.35-3.27$ (m, 2H). ${ }^{13} \mathrm{C}\left\{{ }^{1} \mathrm{H}\right\} \mathrm{NMR}\left(125 \mathrm{MHz}, \mathrm{CDCl}_{3}\right) \delta 193.4,151.5,142.7,140.4,133.7,130.4,129.0,127.5,125.3$, 110.5, 107.7, 43.8, 38.2. HRMS (ESI-ion trap) $m / z$ : $[\mathrm{M}+\mathrm{H}]^{+}$calcd for $\mathrm{C}_{13} \mathrm{H}_{11} \mathrm{O}_{2} \mathrm{~S}, 231.0474$; found 231.0471.

\section{2-(thiophen-2-yl)thiochroman-4-one (3al)}

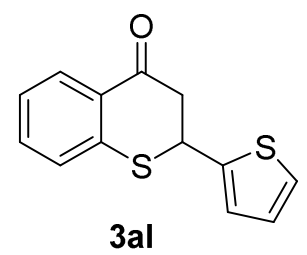

Yellow solid (18.1 mg, 49\% yield); mp $69-71{ }^{\circ} \mathrm{C} ;[\alpha]_{\mathrm{D}}^{25}=-1.0\left(c 1.28, \mathrm{CHCl}_{3}\right)$; ee was determined to be $2 \%$ by HPLC analysis with a Chiralcel OJ-3 column (hexane/2-propanol 60:40, $1.0 \mathrm{~mL} / \mathrm{min}, 254 \mathrm{~nm}) ; \mathrm{t}_{\mathrm{r}}(\operatorname{minor})=12.3$ $\min , \mathrm{t}_{\mathrm{r}}($ major $)=19.2 \mathrm{~min} ;{ }^{1} \mathrm{H} \mathrm{NMR}\left(500 \mathrm{MHz}, \mathrm{CDCl}_{3}\right) \delta 8.14(\mathrm{dd}, J=7.9,1.2 \mathrm{~Hz}, 1 \mathrm{H}), 7.42(\mathrm{td}, J=8.0,1.4 \mathrm{~Hz}$, 1H), 7.29-7.27 (m, 2H), 7.22 (t, $J=7.6 \mathrm{~Hz}, 1 \mathrm{H}), 7.06$ (d, $J=3.5 \mathrm{~Hz}, 1 \mathrm{H}), 6.96$ (dd, $J=5.1,3.6 \mathrm{~Hz}, 1 \mathrm{H}), 4.98$ (dd, $J=10.5,4.2 \mathrm{~Hz}, 1 \mathrm{H}), 3.39-3.31(\mathrm{~m}, 2 \mathrm{H}) .{ }^{13} \mathrm{C}\left\{{ }^{1} \mathrm{H}\right\} \mathrm{NMR}\left(125 \mathrm{MHz}, \mathrm{CDCl}_{3}\right) \delta 193.6,142.0,141.1,133.8,130.4$, 129.2, 127.3, 127.0, 125.8, 125.5, 125.5, 47.5, 40.6. HRMS (ESI-ion trap) $m / z$ : $[\mathrm{M}+\mathrm{H}]^{+}$calcd for $\mathrm{C}_{13} \mathrm{H}_{11} \mathrm{OS}_{2}$, 247.0246; found 231.0241.

\section{6-methoxy-2-phenylthiochroman-4-one (3ba)}<smiles>COc1ccc2c(c1)C(=O)CC(c1ccccc1)S2</smiles>

Light yellow solid (34.0 mg, 84\% yield); mp $102-104{ }^{\circ} \mathrm{C} ;[\alpha]_{\mathrm{D}}^{25}=-145.6\left(c\right.$ 1.07, $\left.\mathrm{CHCl}_{3}\right)$; ee was determined to be $94 \%$ by HPLC analysis with a Chiralcel OJ-3 column (hexane/2-propanol 60:40, $1.0 \mathrm{~mL} / \mathrm{min}, 254 \mathrm{~nm}) ; \mathrm{t}_{\mathrm{r}}($ minor $)=15.2 \mathrm{~min}, \mathrm{t}_{\mathrm{r}}($ major $)=26.8 \mathrm{~min} ;{ }^{1} \mathrm{H} \mathrm{NMR}\left(400 \mathrm{MHz}, \mathrm{CDCl}_{3}\right) \delta$ $7.66(\mathrm{~d}, J=2.9 \mathrm{~Hz}, 1 \mathrm{H}), 7.44-7.32(\mathrm{~m}, 5 \mathrm{H}), 7.20(\mathrm{~d}, J=8.7 \mathrm{~Hz}, 1 \mathrm{H}), 7.05(\mathrm{dd}, J=8.7,2.9 \mathrm{~Hz}, 1 \mathrm{H})$, $4.69(\mathrm{dd}, J=12.9,3.1 \mathrm{~Hz}, 1 \mathrm{H}), 3.84(\mathrm{~s}, 3 \mathrm{H}), 3.31(\mathrm{dd}, J=16.6,12.9 \mathrm{~Hz}, 1 \mathrm{H}), 3.21(\mathrm{dd}, J=16.6,3.2$ $\mathrm{Hz}, 1 \mathrm{H}) .{ }^{13} \mathrm{C}\left\{{ }^{1} \mathrm{H}\right\} \mathrm{NMR}\left(100 \mathrm{MHz}, \mathrm{CDCl}_{3}\right) \delta 194.4,157.6,138.5,133.5,131.2,128.9,128.5,128.4$, 
127.4, 122.6, 111.3, 55.6, 46.7, 45.7; HRMS (ESI-ion trap) $m / z:[\mathrm{M}+\mathrm{H}]^{+}$calcd for $\mathrm{C}_{16} \mathrm{H}_{15} \mathrm{O}_{2} \mathrm{~S}$, 271.0787; found 271.0781.

\section{6-methyl-2-phenylthiochroman-4-one (3ca)}<smiles>Cc1ccc2c(c1)C(=O)CC(c1ccccc1)S2</smiles>

White solid (31.6 mg, 83\% yield); mp 89-91 ${ }^{\circ} \mathrm{C} ;[\alpha]_{\mathrm{D}}^{25}=-121.5\left(c 0.27, \mathrm{CHCl}_{3}\right)$; ee was determined to be $95 \%$ by HPLC analysis with a Chiralcel OJ-3 column (hexane/2-propanol $60: 40,1.0 \mathrm{~mL} / \mathrm{min}$, $254 \mathrm{~nm}) ; \mathrm{t}_{\mathrm{r}}($ minor $)=11.5 \mathrm{~min}, \mathrm{t}_{\mathrm{r}}($ major $)=21.7 \mathrm{~min} ;{ }^{1} \mathrm{H} \mathrm{NMR}\left(500 \mathrm{MHz}, \mathrm{CDCl}_{3}\right) \delta 7.96(\mathrm{~s}, 1 \mathrm{H})$, 7.37 (ddd, $J=23.2,16.6,7.2 \mathrm{~Hz}, 5 \mathrm{H}), 7.25-7.23(\mathrm{~m}, 1 \mathrm{H}), 7.18(\mathrm{~d}, J=8.0 \mathrm{~Hz}, 1 \mathrm{H}), 4.69(\mathrm{dd}, J=$ 13.1, $2.7 \mathrm{~Hz}, 1 \mathrm{H}), 3.30$ (dd, $J=16.3,13.2 \mathrm{~Hz}, 1 \mathrm{H}), 3.19$ (dd, $J=16.4,2.7 \mathrm{~Hz}, 1 \mathrm{H}), 2.35$ (s, 3H). ${ }^{13} \mathrm{C}\left\{{ }^{1} \mathrm{H}\right\}$ NMR $\left(125 \mathrm{MHz}, \mathrm{CDCl}_{3}\right) \delta 194.7,138.7,138.5,135.1,134.8,130.1,129.3,128.9,128.4$, 127.4, 127.1, 46.8, 45.5, 20.8; HRMS (ESI-ion trap) $m / z$ : $[\mathrm{M}+\mathrm{H}]^{+}$calcd for $\mathrm{C}_{16} \mathrm{H}_{15} \mathrm{OS}, 255.0838$; found 255.0838 .

\section{6-fluoro-2-phenylthiochroman-4-one (3da)}<smiles>O=C1CC(c2ccccc2)Sc2ccc(F)cc21</smiles>

Yellow solid (34.0 mg, 88\% yield); mp 64-66 ${ }^{\circ} \mathrm{C} ;[\alpha]_{\mathrm{D}}^{25}=-146.7\left(c 1.03, \mathrm{CHCl}_{3}\right)$; ee was determined to be $93 \%$ by HPLC analysis with a Chiralcel OJ-3 column (hexane/2-propanol 60:40, $1.0 \mathrm{~mL} / \mathrm{min}$, $254 \mathrm{~nm}) ; \mathrm{t}_{\mathrm{r}}($ minor $)=12.7 \mathrm{~min}, \mathrm{t}_{\mathrm{r}}($ major $)=25.6 \mathrm{~min} ;{ }^{1} \mathrm{H} \mathrm{NMR}\left(400 \mathrm{MHz}, \mathrm{CDCl}_{3}\right) \delta 7.82(\mathrm{dd}, J=$ 9.3, $2.9 \mathrm{~Hz}, 1 \mathrm{H}), 7.42-7.33(\mathrm{~m}, 5 \mathrm{H}), 7.24(\mathrm{dd}, J=5.5,3.2 \mathrm{~Hz}, 1 \mathrm{H}), 7.17-7.12(\mathrm{~m}, 1 \mathrm{H}), 4.69(\mathrm{dd}, J=$ 12.8, 3.3 Hz, 1H), $3.25(\mathrm{qd}, J=16.6,8.1 \mathrm{~Hz}, 2 \mathrm{H}) .{ }^{13} \mathrm{C}\left\{{ }^{1} \mathrm{H}\right\} \mathrm{NMR}\left(100 \mathrm{MHz}, \mathrm{CDCl}_{3}\right) \delta 193.3,160.5$ $\left(\mathrm{d}, J_{\mathrm{F}-\mathrm{C}}=245.0 \mathrm{~Hz}\right), 138.0,137.2,\left(\mathrm{~d}, J_{\mathrm{F}-\mathrm{C}}=3.0 \mathrm{~Hz}\right), 131.7\left(\mathrm{~d}, J_{\mathrm{F}-\mathrm{C}}=5.0 \mathrm{~Hz}\right), 129.0,129.0\left(\mathrm{~d}, J_{\mathrm{F}-\mathrm{C}}=\right.$ $7.0 \mathrm{~Hz}), 128.5,127.3,121.5\left(\mathrm{~d}, J_{\mathrm{F}-\mathrm{C}}=23.0 \mathrm{~Hz}\right), 115.1\left(\mathrm{~d}, J_{\mathrm{F}-\mathrm{C}}=23.0 \mathrm{~Hz}\right), 46.3,45.5 .{ }^{19} \mathrm{~F} \mathrm{NMR}(376$ $\left.\mathrm{MHz}, \mathrm{CDCl}_{3}\right) \delta$-116.00; HRMS (ESI-ion trap) $m / z$ : $[\mathrm{M}+\mathrm{H}]^{+}$calcd for $\mathrm{C}_{15} \mathrm{H}_{12} \mathrm{FOS}, 259.0587$; found 259.0580 . 


\section{6-chloro-2-phenylthiochroman-4-one (3ea)}<smiles>O=C1CC(c2ccccc2)Sc2ccc(Cl)cc21</smiles>

Yellow solid (33.2 mg, 81\% yield); $\mathrm{mp} 68-70{ }^{\circ} \mathrm{C} ;[\alpha]_{\mathrm{D}}^{25}=142.68\left(\mathrm{c} 1.07, \mathrm{CHCl}_{3}\right)$; ee was determined to be $97 \%$ by HPLC analysis with a Chiralcel OJ-3 column (hexane/2-propanol 60:40, $1.0 \mathrm{~mL} / \mathrm{min}$, $254 \mathrm{~nm}) ; \mathrm{t}_{\mathrm{r}}($ minor $)=12.6 \mathrm{~min}, \mathrm{t}_{\mathrm{r}}($ major $)=23.0 \mathrm{~min} ;{ }^{1} \mathrm{H} \mathrm{NMR}\left(400 \mathrm{MHz}, \mathrm{CDCl}_{3}\right) \delta 8.11(\mathrm{~d}, J=2.4$ $\mathrm{Hz}, 1 \mathrm{H}), 7.43-7.34(\mathrm{~m}, 6 \mathrm{H}), 7.23$ (d, $J=8.4 \mathrm{~Hz}, 1 \mathrm{H}), 4.70(\mathrm{dd}, J=12.7,3.2 \mathrm{~Hz}, 1 \mathrm{H}), 3.26(\mathrm{ddd}, J=$ 19.8, 16.5, 8.0 Hz, 2H). ${ }^{13} \mathrm{C}\left\{{ }^{1} \mathrm{H}\right\} \mathrm{NMR}\left(100 \mathrm{MHz}, \mathrm{CDCl}_{3}\right) \delta 193.2,140.4,138.0,133.6,131.4,131.4$, 129.0, 128.8, 128.6, 127.4, 46.3, 45.5; HRMS (ESI-ion trap) $m / z:[\mathrm{M}+\mathrm{H}]^{+}$calcd for $\mathrm{C}_{15} \mathrm{H}_{12} \mathrm{ClOS}$, 275.0292; found 275.0287.

\section{6-bromo-2-phenylthiochroman-4-one (3fa)}<smiles>O=C1CC(c2ccccc2)Sc2ccc(Br)cc21</smiles>

Yellow solid (36.9 mg, 77\% yield); $\mathrm{mp} 76-78^{\circ} \mathrm{C} ;[\alpha]_{\mathrm{D}}^{25}=-121.1\left(c 0.73, \mathrm{CHCl}_{3}\right)$; ee was determined to be $96 \%$ by HPLC analysis with a Chiralcel OJ-3 column (hexane $/ 2$-propanol 60:40, $1.0 \mathrm{~mL} / \mathrm{min}$, $254 \mathrm{~nm}) ; \mathrm{t}_{\mathrm{r}}($ minor $)=13.5 \mathrm{~min}, \mathrm{t}_{\mathrm{r}}($ major $)=24.4 \mathrm{~min} ;{ }^{1} \mathrm{H} \mathrm{NMR}\left(500 \mathrm{MHz}, \mathrm{CDCl}_{3}\right) \delta 8.25(\mathrm{~s}, 1 \mathrm{H})$, $7.50(\mathrm{~d}, J=8.4 \mathrm{~Hz}, 1 \mathrm{H}), 7.42-7.33(\mathrm{~m}, 5 \mathrm{H}), 7.16(\mathrm{~d}, J=8.4 \mathrm{~Hz}, 1 \mathrm{H}), 4.71-4.68(\mathrm{~m}, 1 \mathrm{H}), 3.32-3.18$ (m, 2H). ${ }^{13} \mathrm{C}\left\{{ }^{1} \mathrm{H}\right\}$ NMR $\left(125 \mathrm{MHz}, \mathrm{CDCl}_{3}\right) \delta$ 193.1, 141.0, 137.9, 136.3, 131.8, 131.6, 129.0, 128.8, 128.6, 127.4, 118.9, 46.2, 45.4; HRMS (ESI-ion trap) $m / z$ : $[\mathrm{M}+\mathrm{H}]^{+}$calcd for $\mathrm{C}_{15} \mathrm{H}_{12} \mathrm{BrOS}, 318.9787$; found 318.9789 .

\section{2-phenyl-6-(trifluoromethyl)thiochroman-4-one (3ga)}<smiles>O=C1CC(c2ccccc2)Sc2ccc(C(F)(F)F)cc21</smiles>

Yellow solid (23.1 mg, 50\% yield); mp 89-91 ${ }^{\circ} \mathrm{C} ;[\alpha]_{\mathrm{D}}^{25}=-134.6\left(c 0.67, \mathrm{CHCl}_{3}\right)$; ee was determined to be $91 \%$ by HPLC analysis with a Chiralcel OJ-3 column (hexane/2-propanol $60: 40,1.0 \mathrm{~mL} / \mathrm{min}$, $254 \mathrm{~nm}) ; \mathrm{t}_{\mathrm{r}}($ minor $)=15.0 \mathrm{~min}, \mathrm{t}_{\mathrm{r}}($ major $)=26.8 \mathrm{~min} ;{ }^{1} \mathrm{H} \mathrm{NMR}\left(500 \mathrm{MHz}, \mathrm{CDCl}_{3}\right) \delta 8.41-8.40(\mathrm{~m}$, 
$1 \mathrm{H}), 7.63(\mathrm{dd}, J=8.3,1.8 \mathrm{~Hz}, 1 \mathrm{H}), 7.44-7.35(\mathrm{~m}, 6 \mathrm{H}), 4.76(\mathrm{dd}, J=12.9,3.1 \mathrm{~Hz}, 1 \mathrm{H}), 3.35(\mathrm{dd}, J=$ 16.4, $13.0 \mathrm{~Hz}, 1 \mathrm{H}), 3.26(\mathrm{dd}, J=16.4,3.1 \mathrm{~Hz}, 1 \mathrm{H}) .{ }^{13} \mathrm{C}\left\{{ }^{1} \mathrm{H}\right\} \mathrm{NMR}\left(125 \mathrm{MHz}, \mathrm{CDCl}_{3}\right) \delta 193.1,146.6$, $137.7,130.3,129.6\left(\mathrm{q}, J_{\mathrm{F}-\mathrm{C}}=3.75 \mathrm{~Hz}\right), 129.1,128.7,127.8,127.8,127.5,127.4,126.3\left(\mathrm{q}, J_{\mathrm{F}-\mathrm{C}}=3.8\right.$

$\mathrm{Hz}), 124.0\left(\mathrm{~d}, J_{\mathrm{F}-\mathrm{C}}=270.0 \mathrm{~Hz}\right), 46.1,45.4 .{ }^{19} \mathrm{~F}$ NMR $\left(376 \mathrm{MHz}, \mathrm{CDCl}_{3}\right) \delta-62.80$; HMS (ESI-ion trap) $m / z:[\mathrm{M}-\mathrm{H}]^{-}$calcd for $\mathrm{C}_{16} \mathrm{H}_{10} \mathrm{~F}_{3} \mathrm{OS}, 307.0410$; found 307.0413 .

\section{5-methyl-2-phenylthiochroman-4-one (3ha)}

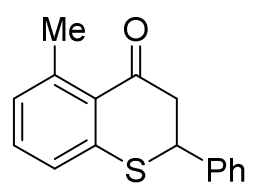

White solid (28.0 mg, 74\% yield); $\mathrm{mp} 95-97{ }^{\circ} \mathrm{C} ;[\alpha]_{\mathrm{D}}^{25}=-121.8\left(\mathrm{c} 0.38, \mathrm{CHCl}_{3}\right)$; ee was determined to be $96 \%$ by HPLC analysis with a Chiralcel OJ-3 column (hexane/2-propanol 60:40, $1.0 \mathrm{~mL} / \mathrm{min}$, $254 \mathrm{~nm}) ; \mathrm{t}_{\mathrm{r}}($ minor $)=11.4 \mathrm{~min}, \mathrm{t}_{\mathrm{r}}($ major $)=17.7 \mathrm{~min} ;{ }^{1} \mathrm{H} \mathrm{NMR}\left(500 \mathrm{MHz}, \mathrm{CDCl}_{3}\right) \delta 7.37(\mathrm{ddd}, J=$ 24.6, 15.9, 7.3 Hz, 5H), $7.23(\mathrm{~d}, J=7.7 \mathrm{~Hz}, 1 \mathrm{H}), 7.16$ (d, $J=7.8 \mathrm{~Hz}, 1 \mathrm{H}), 7.00(\mathrm{~d}, J=7.5 \mathrm{~Hz}, 1 \mathrm{H})$, $4.69(\mathrm{dd}, J=13.2,2.6 \mathrm{~Hz}, 1 \mathrm{H}), 3.35$ (dd, $J=15.4,13.6 \mathrm{~Hz}, 1 \mathrm{H}), 3.19$ (dd, $J=15.8,2.7 \mathrm{~Hz}, 1 \mathrm{H})$, 2.64 (s, 3H). ${ }^{13} \mathrm{C}\left\{{ }^{1} \mathrm{H}\right\} \operatorname{NMR}\left(125 \mathrm{MHz}, \mathrm{CDCl}_{3}\right) \delta 196.7,143.1,142.8,138.5,132.1,129.5,129.3$, 128.9, 128.3, 127.4, 125.2, 48.7, 44.9, 23.8; HRMS (ESI-ion trap) $m / z:[\mathrm{M}+\mathrm{H}]^{+}$calcd for $\mathrm{C}_{16} \mathrm{H}_{15} \mathrm{OS}$, 255.0838; found 255.0840 .

\section{7-methyl-2-phenylthiochroman-4-one (3ia)}

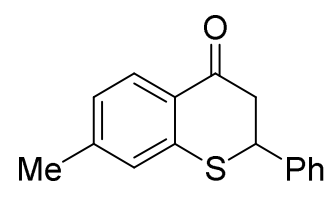

White solid (32.0 mg, 84\% yield); mp $104-106{ }^{\circ} \mathrm{C} ;[\alpha]_{\mathrm{D}}^{25}=-143.8\left(\mathrm{c} 1.00, \mathrm{CHCl}_{3}\right)$; ee was determined to be $94 \%$ by HPLC analysis with a Chiralcel OJ-3 column (hexane/2-propanol 60:40, $1.0 \mathrm{~mL} / \mathrm{min}, 254 \mathrm{~nm}) ; \mathrm{t}_{\mathrm{r}}$ (minor) $=13.5 \mathrm{~min}, \mathrm{t}_{\mathrm{r}}($ major $)=24.3 \mathrm{~min} ;{ }^{1} \mathrm{H} \mathrm{NMR}\left(500 \mathrm{MHz}, \mathrm{CDCl}_{3}\right) \delta$ $8.05(\mathrm{~d}, J=8.1 \mathrm{~Hz}, 1 \mathrm{H}), 7.37$ (ddd, $J=30.9,15.8,7.3 \mathrm{~Hz}, 5 \mathrm{H}), 7.09$ (s, 1H), 7.01 (d, $J=8.1 \mathrm{~Hz}, 1 \mathrm{H})$, $4.70(\mathrm{dd}, J=13.0,2.3 \mathrm{~Hz}, 1 \mathrm{H}), 3.29$ (dd, $J=16.3,13.2 \mathrm{~Hz}, 1 \mathrm{H}), 3.17$ (dd, $J=16.4,2.4 \mathrm{~Hz}, 1 \mathrm{H})$, $2.34(\mathrm{~s}, 3 \mathrm{H}) .{ }^{13} \mathrm{C}\left\{{ }^{1} \mathrm{H}\right\} \mathrm{NMR}\left(125 \mathrm{MHz}, \mathrm{CDCl}_{3}\right) \delta 194.1,144.8,142.0,138.5,129.2,128.9,128.4$, 128.1, 127.4, 127.3, 126.5, 46.6, 45.5, 21.6; HRMS (ESI-ion trap) $m / z:[\mathrm{M}+\mathrm{H}]^{+}$calcd for $\mathrm{C}_{16} \mathrm{H}_{15} \mathrm{OS}$, 
255.0838; found 255.0837 .

\section{8-methyl-2-phenylthiochroman-4-one (3ja)}<smiles>Cc1cccc2c1SC(c1ccccc1)CC2=O</smiles>

White solid (23.1 mg, 61\% yield); mp $112-114{ }^{\circ} \mathrm{C} ;[\alpha]_{\mathrm{D}}^{25}=-130.1\left(c \quad 0.52, \mathrm{CHCl}_{3}\right)$; ee was determined to be $89 \%$ by HPLC analysis with a Chiralcel OJ-3 column (hexane/2-propanol 60:40, $1.0 \mathrm{~mL} / \mathrm{min}, 254 \mathrm{~nm}) ; \mathrm{t}_{\mathrm{r}}($ minor $)=12.4 \mathrm{~min}, \mathrm{t}_{\mathrm{r}}($ major $)=16.6 \mathrm{~min} ;{ }^{1} \mathrm{H} \mathrm{NMR}\left(500 \mathrm{MHz}, \mathrm{CDCl}_{3}\right) \delta$ $8.05(\mathrm{~d}, J=7.9 \mathrm{~Hz}, 1 \mathrm{H}), 7.45-7.31(\mathrm{~m}, 6 \mathrm{H}), 7.12(\mathrm{t}, J=7.6 \mathrm{~Hz}, 1 \mathrm{H}), 4.65(\mathrm{dd}, J=13.5,2.1 \mathrm{~Hz}, 1 \mathrm{H})$, 3.33-3.27 (m, 1H), $3.18(\mathrm{dd}, J=16.1,2.3 \mathrm{~Hz}, 1 \mathrm{H}), 2.30(\mathrm{~s}, 3 \mathrm{H}) .{ }^{13} \mathrm{C}\left\{{ }^{1} \mathrm{H}\right\} \mathrm{NMR}\left(125 \mathrm{MHz}, \mathrm{CDCl}_{3}\right) \delta$ $194.8,141.6,138.5,135.1,134.7,130.5,129.0,128.5,127.5,126.9,124.3,46.1$, 44.8, 19.9; HRMS (ESI-ion trap) $m / z$ : $[\mathrm{M}+\mathrm{H}]^{+}$calcd for $\mathrm{C}_{16} \mathrm{H}_{15} \mathrm{OS}, 255.0838$; found 255.0838.

\section{5-fluoro-2-phenylthiochroman-4-one (3ka)}<smiles>O=C1CC(c2ccccc2)Sc2cccc(F)c21</smiles>

Yellow solid (27.0 mg, 70\% yield); mp 56-58 ${ }^{\circ} \mathrm{C} ;[\alpha]_{\mathrm{D}}^{25}=-134.6\left(c \quad 0.67, \mathrm{CHCl}_{3}\right)$; ee was determined to be $95 \%$ by HPLC analysis with a Chiralcel OJ-3 column (hexane/2-propanol 60:40, $1.0 \mathrm{~mL} / \mathrm{min}$, $254 \mathrm{~nm}) ; \mathrm{t}_{\mathrm{r}}($ minor $)=18.2 \mathrm{~min}, \mathrm{t}_{\mathrm{r}}($ major $)=30.6 \mathrm{~min} ;{ }^{1} \mathrm{H} \mathrm{NMR}\left(400 \mathrm{MHz}, \mathrm{CDCl}_{3}\right) \delta 8.18(\mathrm{dd}, J=$ 8.8, 6.1 Hz, 1H), 7.43-7.35 (m, 5H), 6.98 (dd, $J=8.9,2.4 \mathrm{~Hz}, 1 \mathrm{H}), 6.92-6.88(\mathrm{~m}, 1 \mathrm{H}), 4.74(\mathrm{dd}, J=$ 12.9, 3.1 Hz, 1H), $3.31(\mathrm{dd}, J=16.5,12.9 \mathrm{~Hz}, 1 \mathrm{H}), 3.20(\mathrm{dd}, J=16.5,3.1 \mathrm{~Hz}, 1 \mathrm{H}) .{ }^{13} \mathrm{C}\left\{{ }^{1} \mathrm{H}\right\} \mathrm{NMR}$ $\left(125 \mathrm{MHz}, \mathrm{CDCl}_{3}\right) \delta 193.0,165.5\left(\mathrm{~d}, J_{\mathrm{F}-\mathrm{C}}=255.0 \mathrm{~Hz}\right), 144.9\left(\mathrm{~d}, J_{\mathrm{F}-\mathrm{C}}=10.0 \mathrm{~Hz}\right), 137.9,132.1(\mathrm{~d}$, $\left.J_{\mathrm{F}-\mathrm{C}}=10.0 \mathrm{~Hz}\right), 129.0,128.6,127.4,127.1\left(\mathrm{~d}, J_{\mathrm{F}-\mathrm{C}}=2.5 \mathrm{~Hz}\right), 113.5\left(\mathrm{~d}, J_{\mathrm{F}-\mathrm{C}}=23.8 \mathrm{~Hz}\right), 113.2\left(\mathrm{~d}, J_{\mathrm{F}-\mathrm{C}}\right.$ $=21.3 \mathrm{~Hz}), 46.3,45.7 .{ }^{19} \mathrm{~F}$ NMR $\left(376 \mathrm{MHz}, \mathrm{CDCl}_{3}\right) \delta-103.71$; HRMS (ESI-ion trap) $m / z:[\mathrm{M}+\mathrm{H}]^{+}$ calcd for $\mathrm{C}_{15} \mathrm{H}_{12} \mathrm{FOS}, 259.0587$; found 259.0585.

\section{7-fluoro-2-phenylthiochroman-4-one (3la)}




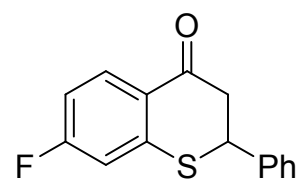

Yellow solid (31.0 mg, 81\% yield); mp $61-63{ }^{\circ} \mathrm{C} ;[\alpha]_{\mathrm{D}}^{25}=-116.5\left(c 0.37, \mathrm{CHCl}_{3}\right)$; ee was determined to be $94 \%$ by HPLC analysis with a Chiralcel OJ-3 column (hexane/2-propanol 60:40, $1.0 \mathrm{~mL} / \mathrm{min}$, $254 \mathrm{~nm}) ; \mathrm{t}_{\mathrm{r}}($ minor $)=9.1 \mathrm{~min}, \mathrm{t}_{\mathrm{r}}($ major $)=11.2 \mathrm{~min} ;{ }^{1} \mathrm{H} \mathrm{NMR}\left(400 \mathrm{MHz}, \mathrm{CDCl}_{3}\right) \delta 8.18(\mathrm{dd}, J=8.8$, $6.1 \mathrm{~Hz}, 1 \mathrm{H}), 7.44-7.35(\mathrm{~m}, 5 \mathrm{H}), 6.98$ (dd, $J=8.9,2.4 \mathrm{~Hz}, 1 \mathrm{H}), 6.90$ (ddd, $J=10.7,8.4,2.5 \mathrm{~Hz}, 1 \mathrm{H})$, $4.74(\mathrm{dd}, J=12.9,3.1 \mathrm{~Hz}, 1 \mathrm{H}), 3.31$ (dd, $J=16.5,12.9 \mathrm{~Hz}, 1 \mathrm{H}), 3.20$ (dd, $J=16.5,3.1 \mathrm{~Hz}, 1 \mathrm{H})$. ${ }^{13} \mathrm{C}\left\{{ }^{1} \mathrm{H}\right\} \mathrm{NMR}\left(125 \mathrm{MHz}, \mathrm{CDCl}_{3}\right) \delta 193.0,165.5\left(\mathrm{~d}, J_{\mathrm{F}-\mathrm{C}}=256.3 \mathrm{~Hz}\right), 144.9\left(\mathrm{~d}, J_{\mathrm{F}-\mathrm{C}}=10.0 \mathrm{~Hz}\right)$, $137.9,132.1\left(\mathrm{~d}, J_{\mathrm{F}-\mathrm{C}}=10.0 \mathrm{~Hz}\right), 129.1,128.6,127.4,127.1\left(\mathrm{~d}, J_{\mathrm{F}-\mathrm{C}}=3.75 \mathrm{~Hz}\right), 113.5\left(\mathrm{~d}, J_{\mathrm{F}-\mathrm{C}}=23.8\right.$ $\mathrm{Hz}), 113.2\left(\mathrm{~d}, J_{\mathrm{F}-\mathrm{C}}=22.5 \mathrm{~Hz}\right), 46.3,45.7 .{ }^{19} \mathrm{~F}$ NMR $\left(376 \mathrm{MHz}, \mathrm{CDCl}_{3}\right) \delta$-103.70; HRMS (ESI-ion trap) $m / z:[\mathrm{M}+\mathrm{H}]^{+}$calcd for $\mathrm{C}_{15} \mathrm{H}_{12} \mathrm{FOS}, 259.0587$; found 259.0584 .

\section{8-fluoro-2-phenylthiochroman-4-one (3ma)}<smiles>O=C1CC(c2ccccc2)Sc2c(F)cccc21</smiles>

Yellow solid (20.3 mg, 52\% yield); mp 62-64 ${ }^{\circ} \mathrm{C} ;[\alpha]_{\mathrm{D}}^{25}=-157.5\left(c 0.27, \mathrm{CHCl}_{3}\right)$; ee was determined to be $83 \%$ by HPLC analysis with a Chiralcel OJ-3 column (hexane/2-propanol 60:40, $1.0 \mathrm{~mL} / \mathrm{min}$, $254 \mathrm{~nm}) ; \mathrm{t}_{\mathrm{r}}($ minor $)=11.2 \mathrm{~min}, \mathrm{t}_{\mathrm{r}}$ (major) $=20.9 \mathrm{~min} ;{ }^{1} \mathrm{H}$ NMR $\left(400 \mathrm{MHz}, \mathrm{CDCl}_{3}\right) \delta 7.98(\mathrm{dd}, J=$ 7.6, $1.4 \mathrm{~Hz}, 1 \mathrm{H}), 7.46-7.35(\mathrm{~m}, 5 \mathrm{H}), 7.25-7.16(\mathrm{~m}, 2 \mathrm{H}), 4.69$ (dd, $J=13.2,2.9 \mathrm{~Hz}, 1 \mathrm{H}), 3.33$ (dd, $J$ $=16.4,13.2 \mathrm{~Hz}, 1 \mathrm{H}), 3.21(\mathrm{dd}, J=16.4,2.7 \mathrm{~Hz}, 1 \mathrm{H}) .{ }^{13} \mathrm{C}\left\{{ }^{1} \mathrm{H}\right\} \mathrm{NMR}\left(100 \mathrm{MHz}, \mathrm{CDCl}_{3}\right) \delta 193.4$, $158.1\left(\mathrm{~d}, J_{\mathrm{F}-\mathrm{C}}=243.0 \mathrm{~Hz}\right), 138.0,132.1\left(\mathrm{~d}, J_{\mathrm{F}-\mathrm{C}}=1.0 \mathrm{~Hz}\right), 130.1\left(\mathrm{~d}, J_{\mathrm{F}-\mathrm{C}}=18.0 \mathrm{~Hz}\right), 129.1,128.7$, $127.5,125.2\left(\mathrm{~d}, J_{\mathrm{F}-\mathrm{C}}=7.0 \mathrm{~Hz}\right), 124.7\left(\mathrm{~d}, J_{\mathrm{F}-\mathrm{C}}=3.0 \mathrm{~Hz}\right), 119.7\left(\mathrm{~d}, J_{\mathrm{F}-\mathrm{C}}=21.0 \mathrm{~Hz}\right), 46.2,44.8 .{ }^{19} \mathrm{~F}$ NMR (376 MHz, $\mathrm{CDCl}_{3}$ ) $\delta-111.41$; HRMS (ESI-ion trap) $m / z:[\mathrm{M}+\mathrm{H}]^{+}$calcd for $\mathrm{C}_{15} \mathrm{H}_{12} \mathrm{FOS}$, 259.0587; found 259.0588 .

\section{6,7-dimethyl-2-phenylthiochroman-4-one (3na)}




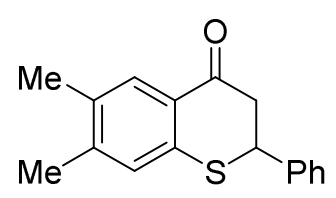

White solid (34.2 mg, 85\% yield); $\mathrm{mp} 108-110{ }^{\circ} \mathrm{C} ;[\alpha]_{\mathrm{D}}^{25}=-221.0$ (c $0.92, \mathrm{CHCl}_{3}$ ); ee was determined to be $95 \%$ by HPLC analysis with a Chiralcel OJ-3 column (hexane/2-propanol 60:40, $1.0 \mathrm{~mL} / \mathrm{min}, 254 \mathrm{~nm}) ; \mathrm{t}_{\mathrm{r}}$ (minor) $=13.4 \mathrm{~min}, \mathrm{t}_{\mathrm{r}}$ (major) $=21.5 \mathrm{~min} ;{ }^{1} \mathrm{H} \mathrm{NMR}\left(400 \mathrm{MHz}, \mathrm{CDCl}_{3}\right) \delta$ $7.91(\mathrm{~s}, 1 \mathrm{H}), 7.43-7.30(\mathrm{~m}, 5 \mathrm{H}), 7.05(\mathrm{~s}, 1 \mathrm{H}), 4.67$ (dd, $J=12.8,3.0 \mathrm{~Hz}, 1 \mathrm{H}), 3.21$ (ddd, $J=19.5$, 16.5, $8.0 \mathrm{~Hz}, 2 \mathrm{H}), 2.26(\mathrm{~s}, 6 \mathrm{H}) .{ }^{13} \mathrm{C}\left\{{ }^{1} \mathrm{H}\right\} \mathrm{NMR}\left(125 \mathrm{MHz}, \mathrm{CDCl}_{3}\right) \delta 194.5,143.9,139.0,138.7$, 134.2, 129.7, 128.9, 128.3, 127.9, 127.4, 46.7, 45.6, 20.00, 19.2; HRMS (ESI-ion trap) $\mathrm{m} / z$ : $[\mathrm{M}+\mathrm{H}]^{+}$ calcd for $\mathrm{C}_{17} \mathrm{H}_{17} \mathrm{OS}, 269.0995$; found 269.0994 .

\section{2-phenyl-2,3-dihydro-4H-benzo[g]thiochromen-4-one (3oa)}<smiles>O=C1CC(c2ccccc2)Sc2cc3ccccc3cc21</smiles>

White solid (25.2 mg, 57\% yield); $\mathrm{mp} 84-86{ }^{\circ} \mathrm{C} ;[\alpha]_{\mathrm{D}}^{25}=-190.5\left(c 0.27, \mathrm{CHCl}_{3}\right)$; ee was determined to be $90 \%$ by HPLC analysis with a Chiralcel OJ-3 column (hexane/2-propanol 60:40, $1.0 \mathrm{~mL} / \mathrm{min}$, $254 \mathrm{~nm}) ; \mathrm{t}_{\mathrm{r}}($ minor $)=20.8 \mathrm{~min}, \mathrm{t}_{\mathrm{r}}($ major $)=39.3 \mathrm{~min} ;{ }^{1} \mathrm{H} \mathrm{NMR}\left(400 \mathrm{MHz}, \mathrm{CDCl}_{3}\right) \delta 9.22(\mathrm{~d}, J=8.7$ Hz, 1H), 7.79 (dd, $J=21.0,8.3 \mathrm{~Hz}, 2 \mathrm{H}), 7.62$ (ddd, $J=8.6,6.9,1.4 \mathrm{~Hz}, 1 \mathrm{H}), 7.49-7.46$ (m, 3H), $7.42-7.33(\mathrm{~m}, 3 \mathrm{H}), 7.29$ (d, $J=8.7 \mathrm{~Hz}, 1 \mathrm{H}), 4.81(\mathrm{dd}, J=13.4,3.1 \mathrm{~Hz}, 1 \mathrm{H}), 3.48$ (dd, $J=15.7,13.4$ $\mathrm{Hz}, 1 \mathrm{H}), 3.31(\mathrm{dd}, J=15.7,3.2 \mathrm{~Hz}, 1 \mathrm{H}) .{ }^{13} \mathrm{C}\left\{{ }^{1} \mathrm{H}\right\} \mathrm{NMR}\left(100 \mathrm{MHz}, \mathrm{CDCl}_{3}\right) \delta 196.4,145.1,138.2$, 134.0, 132.4, 131.9, 129.3, 129.0, 128.5, 128.5, 127.4, 126.0, 125.8, 125.1, 124.9, 48.4, 45.2; HRMS (ESI-ion trap) $m / z$ : $[\mathrm{M}+\mathrm{H}]^{+}$calcd for $\mathrm{C}_{19} \mathrm{H}_{15} \mathrm{OS}, 291.0838$; found 291.0837.

\section{References}

1. (a) Giles, P. R.; Marson, C. M. Aust. J. Chem. 1992, 45, 439-443. (b) Jia, W.; Liu, Y.-J.; Li, W.; Liu, Y.; Zhang, D.-J.; Zhang, P.; Gong, P. Bioorg. Med. Chem. 2009, 17, 4569-4574.

2. Liang, Y.-F.; C. Fu, G. C. J. Am. Chem. Soc. 2014, 136, 5520-5524. 


\section{Copies of ${ }^{1} \mathrm{H},{ }^{13} \mathrm{C}$ and ${ }^{19} \mathrm{~F}$ Spectra}

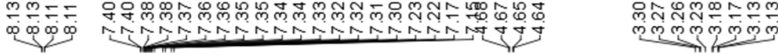
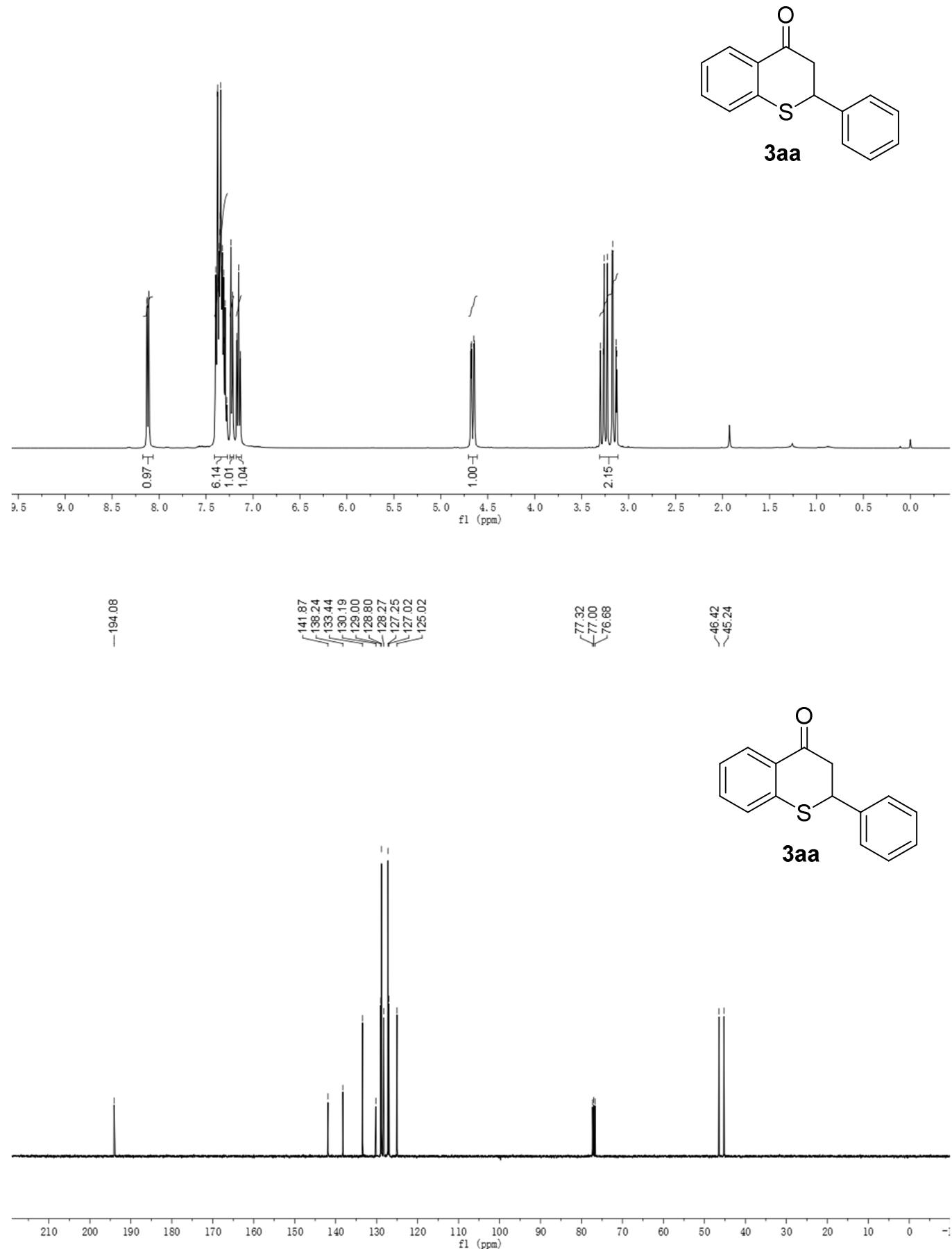

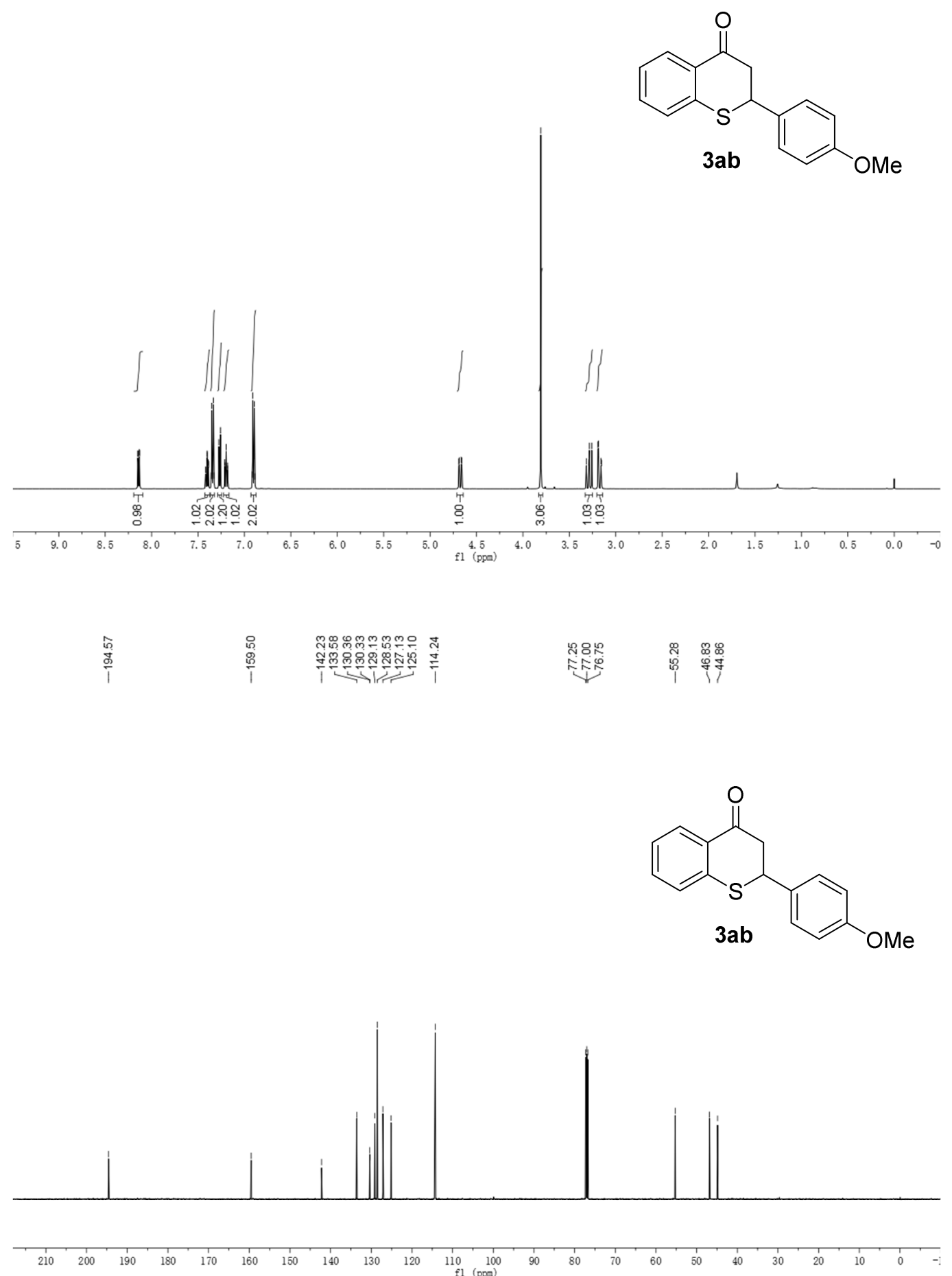

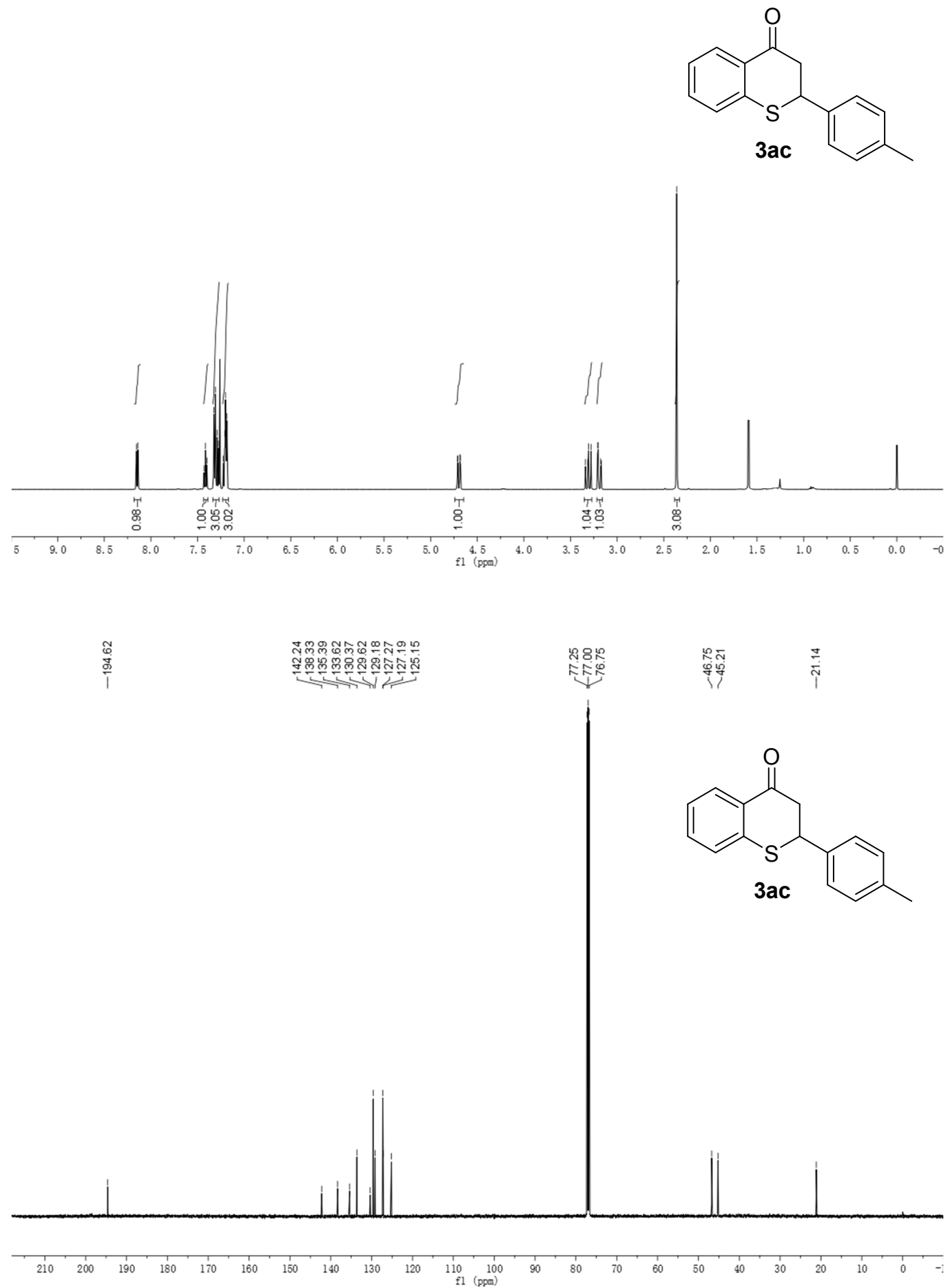

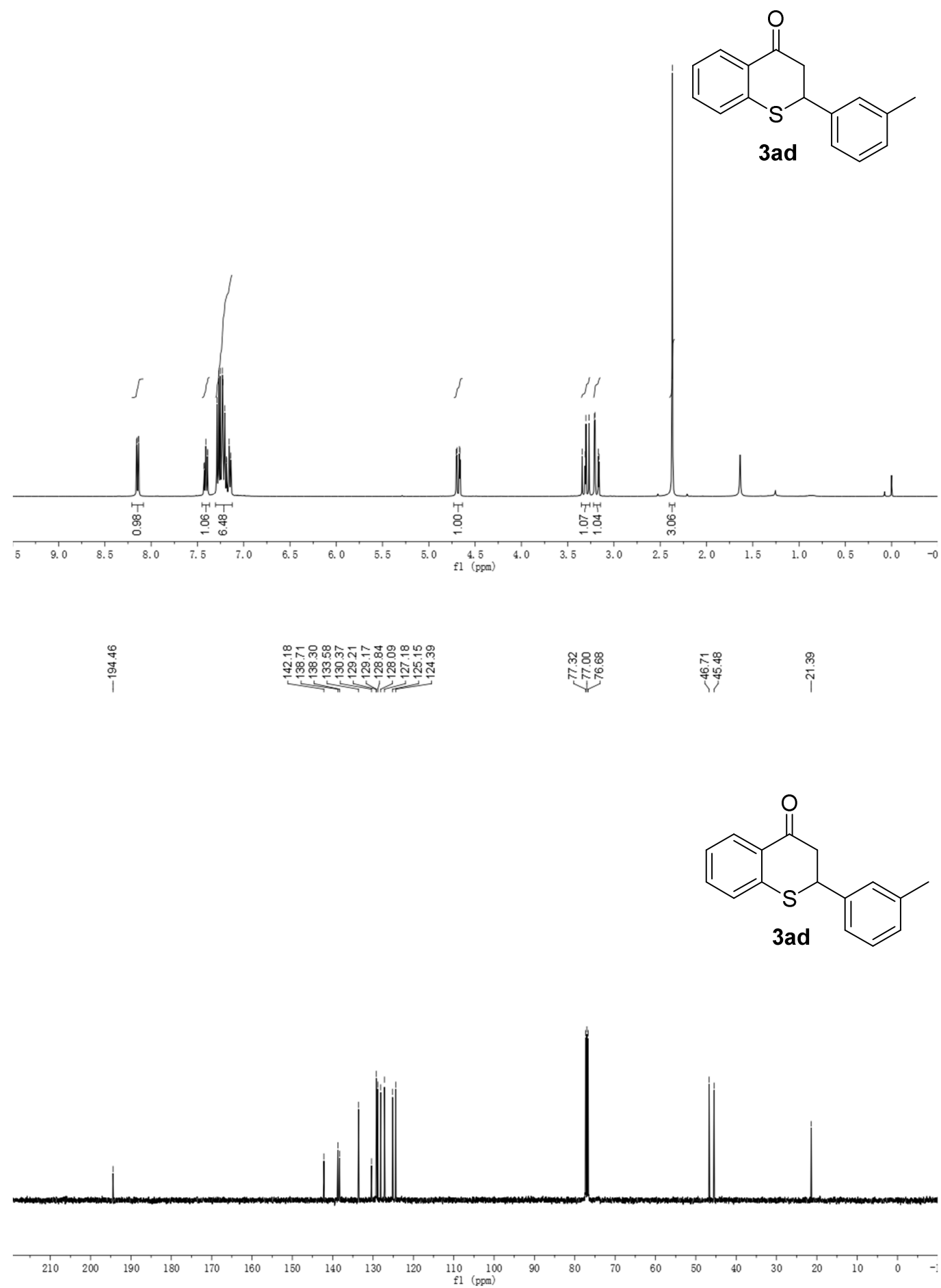

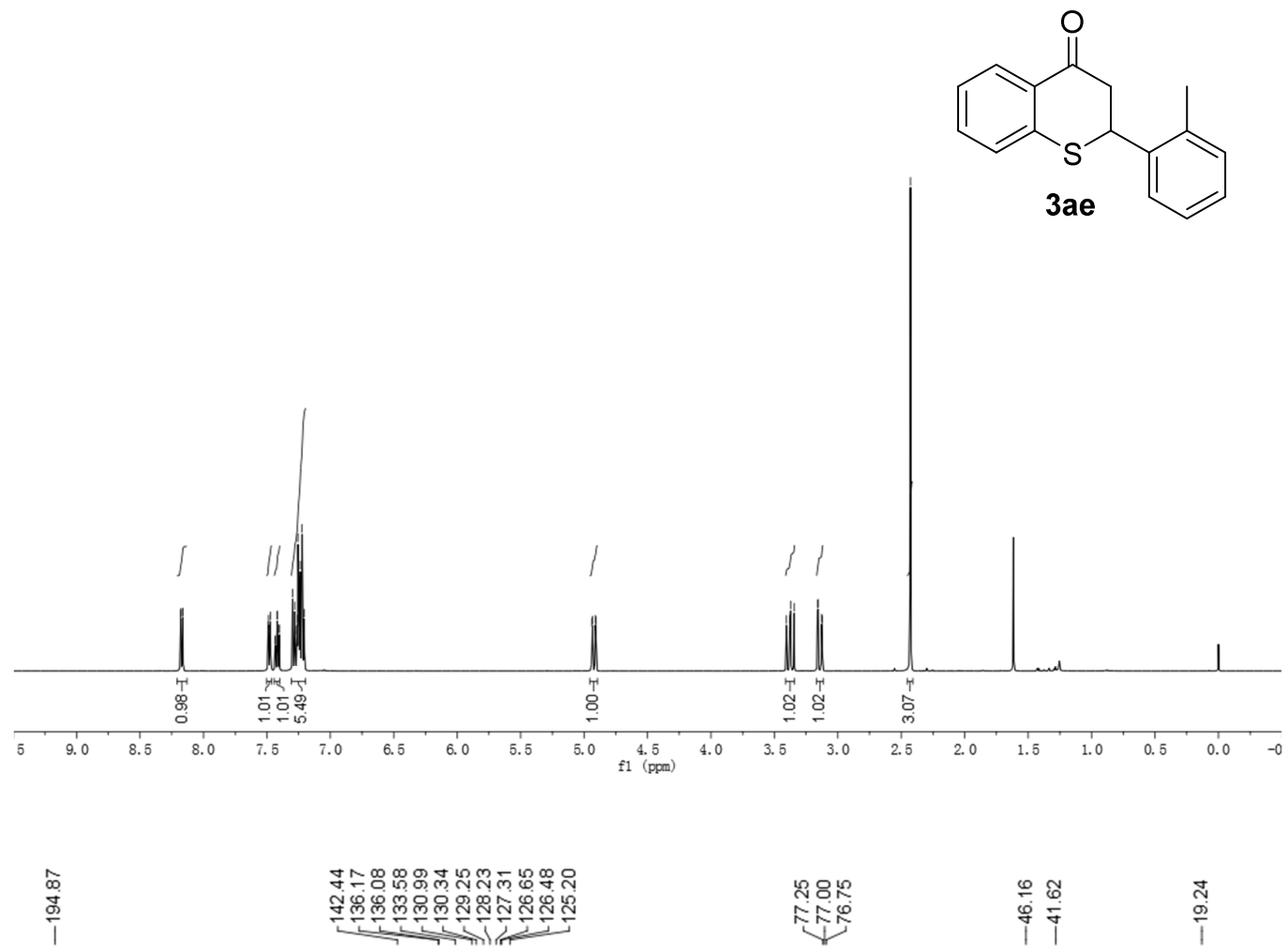

证
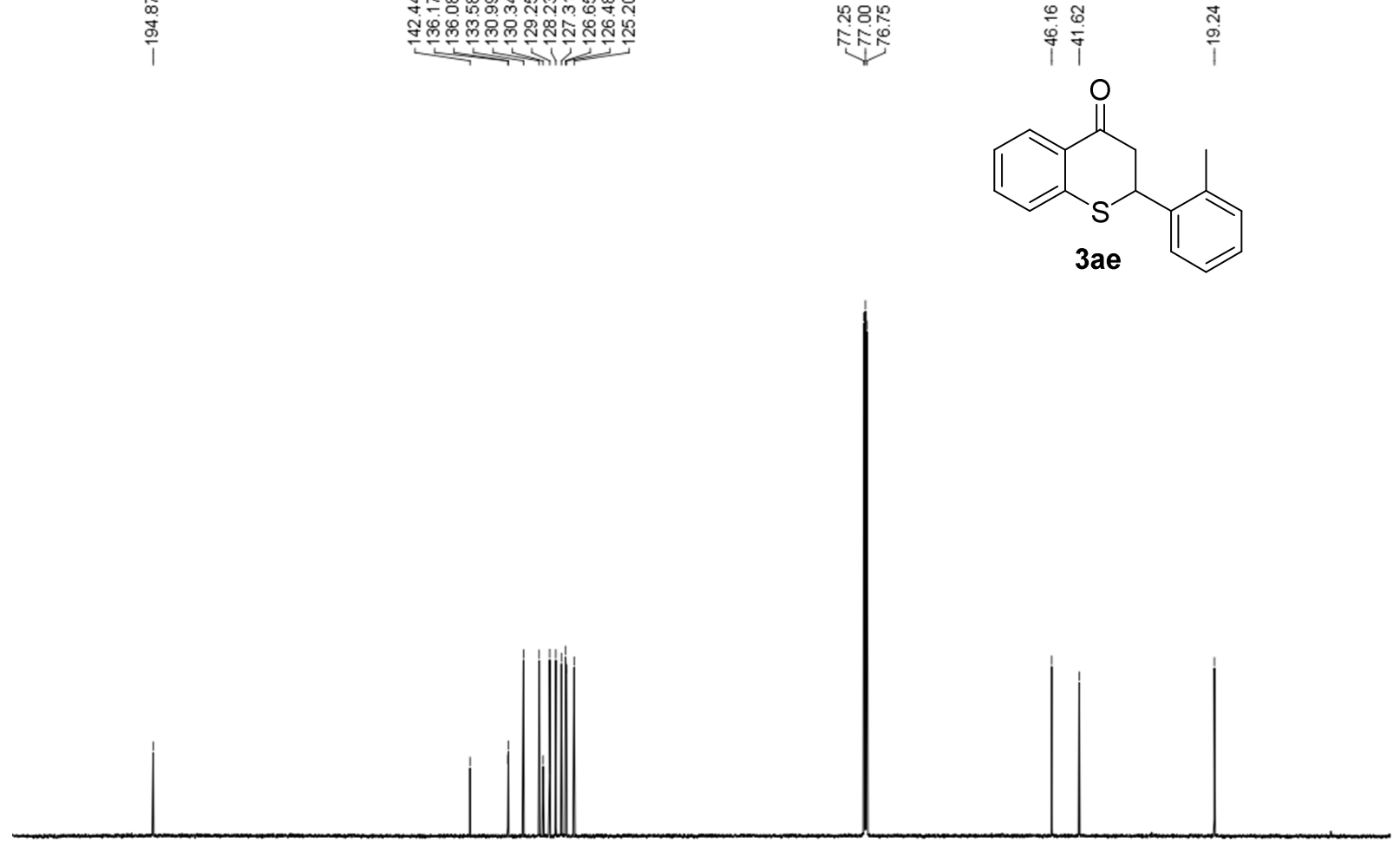

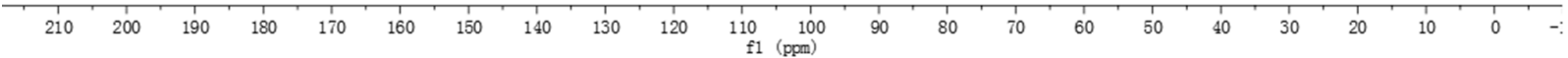



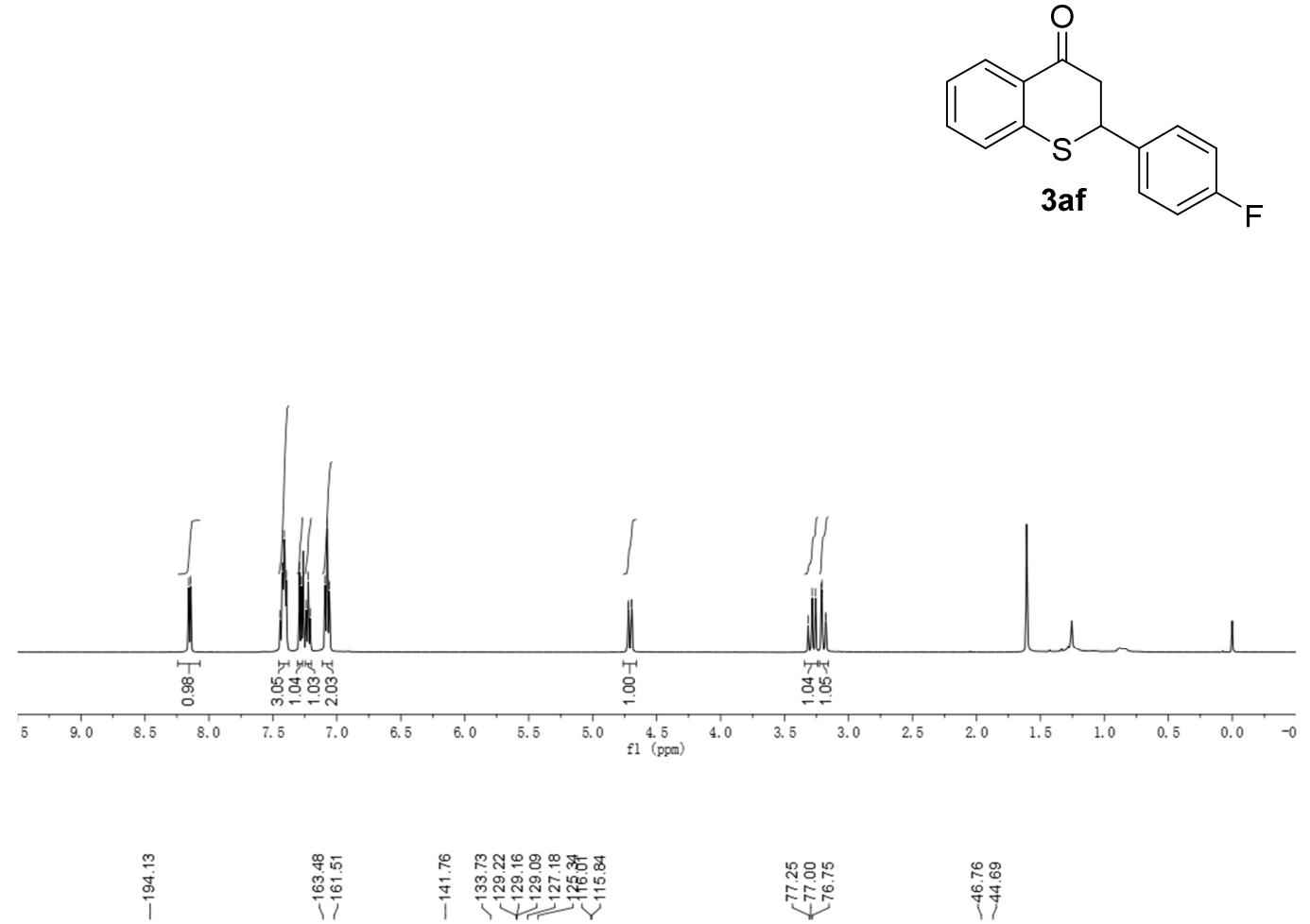

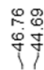

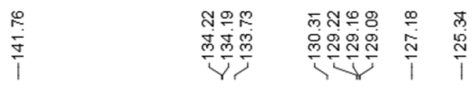
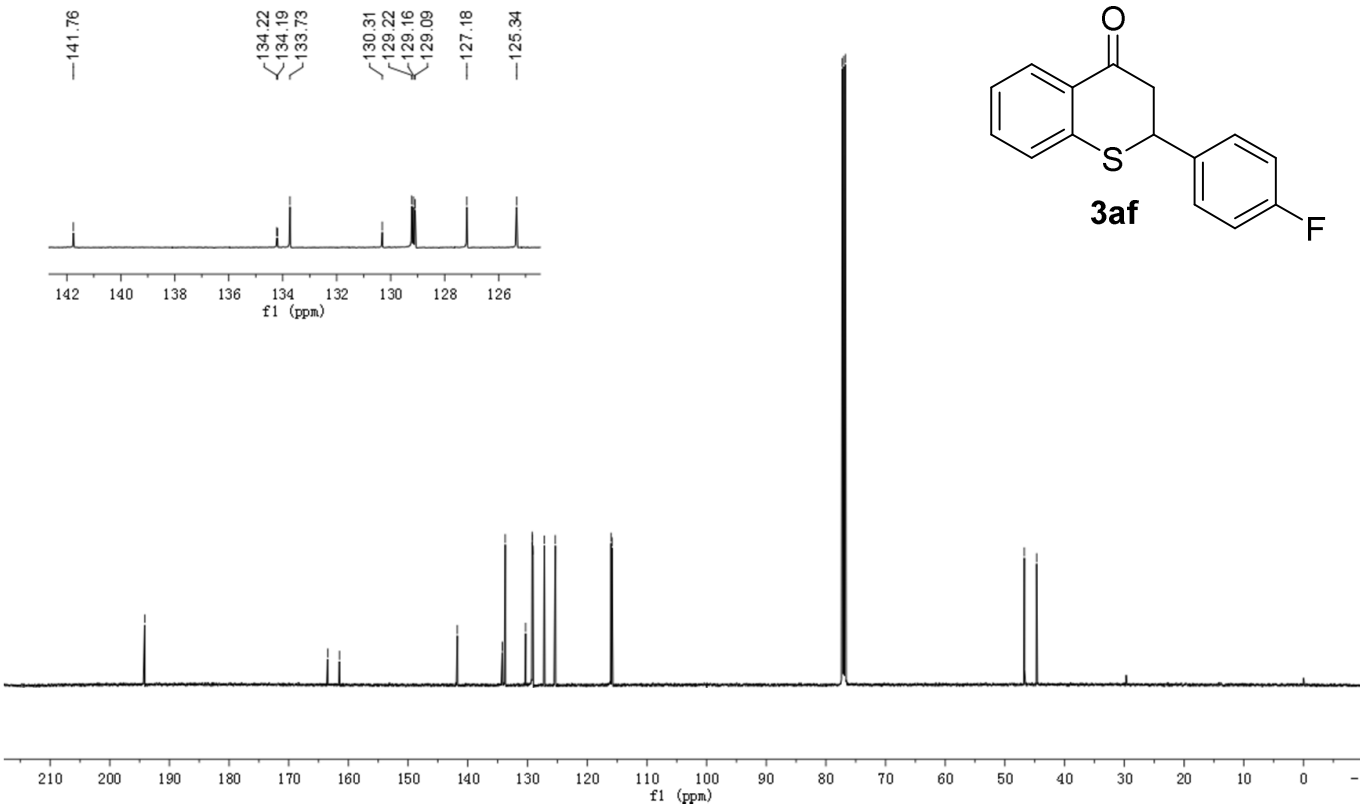

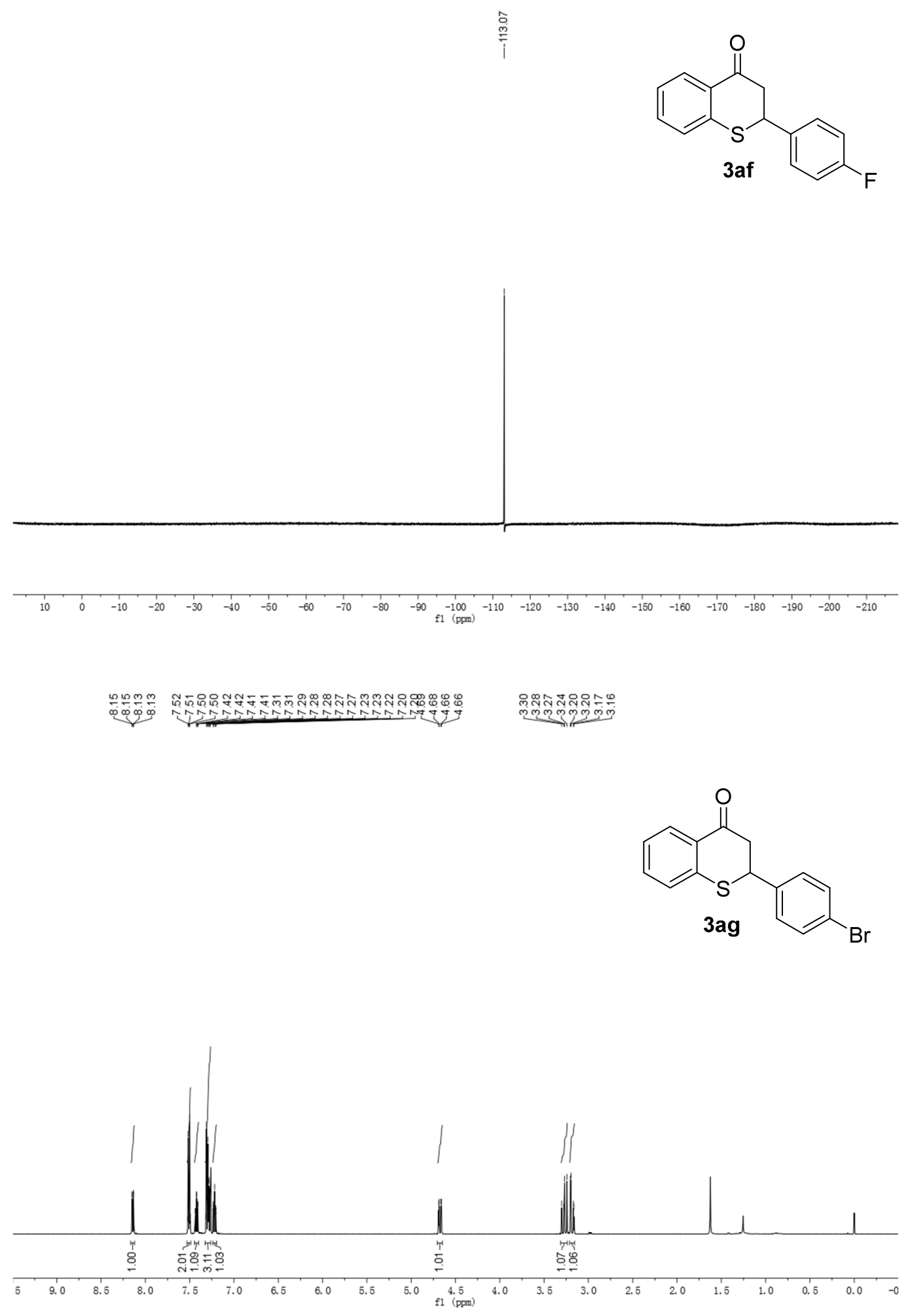


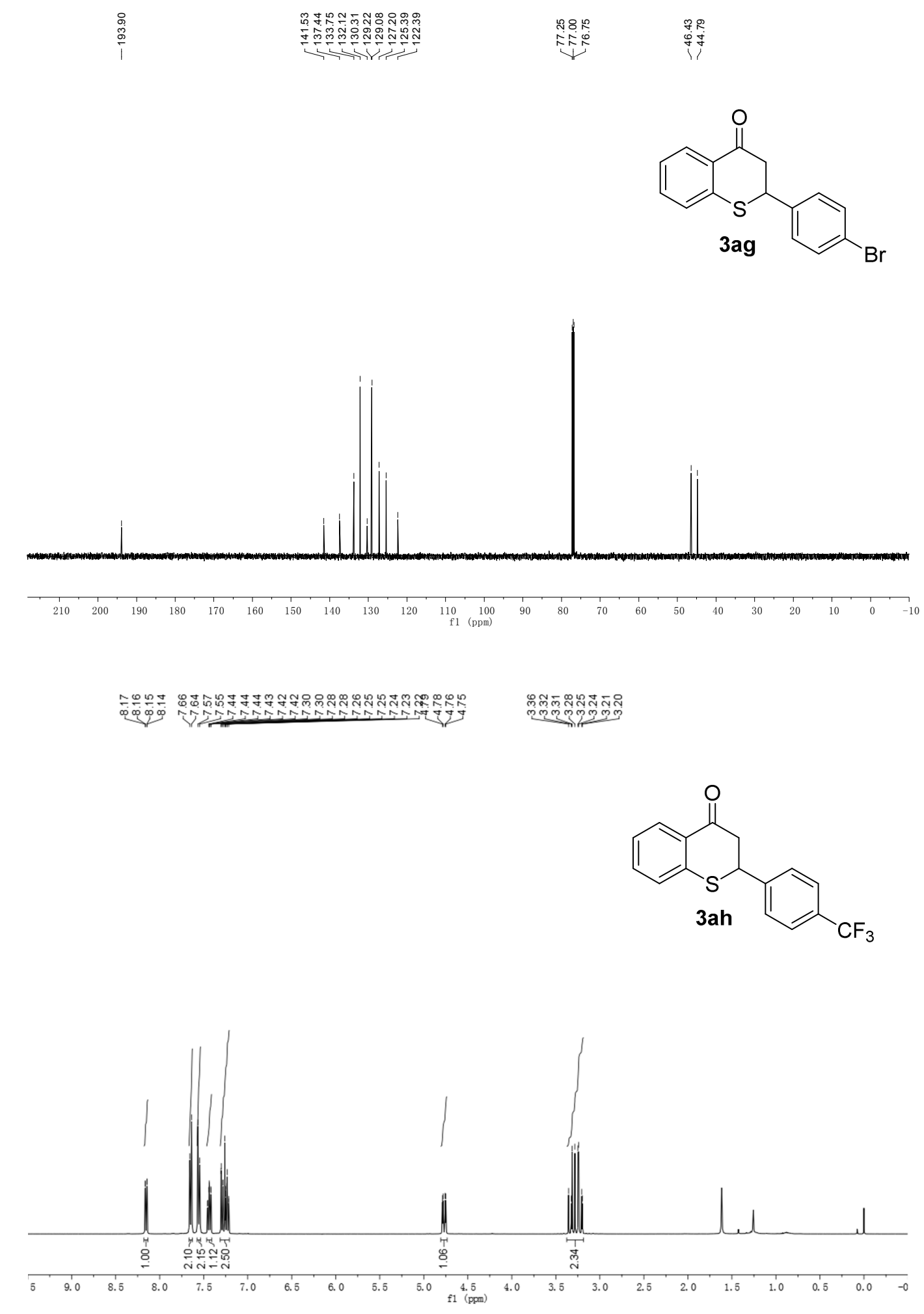



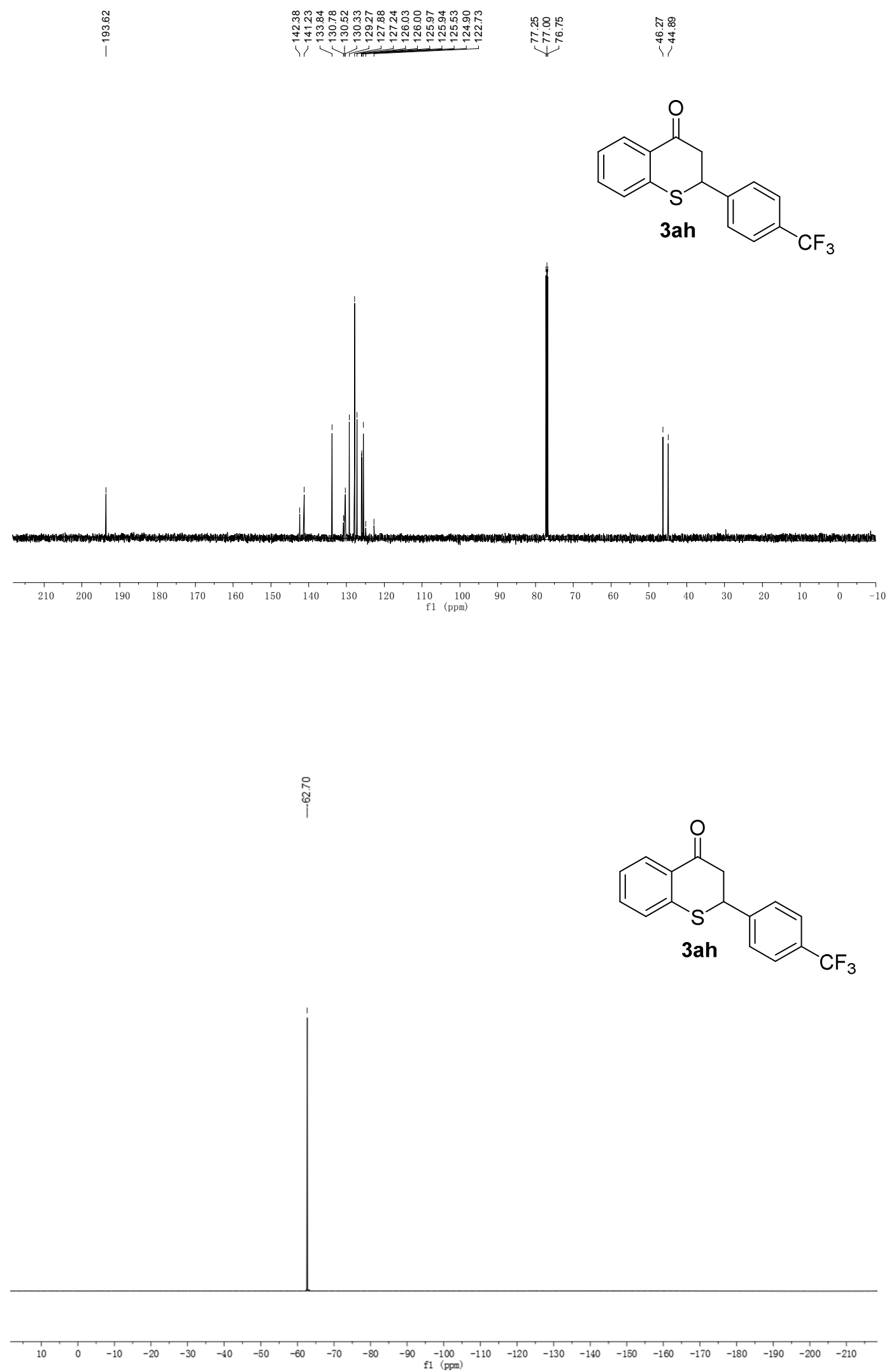

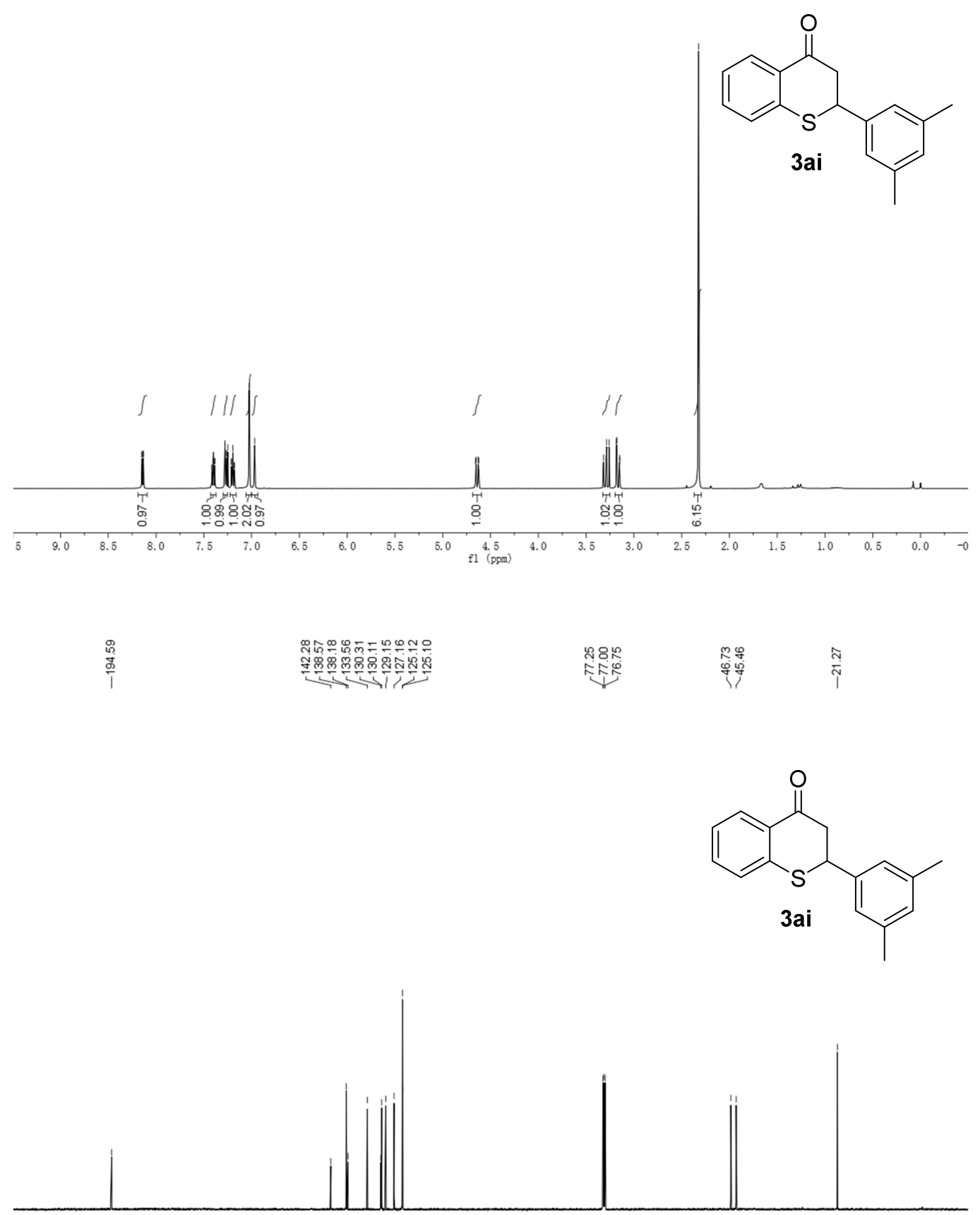

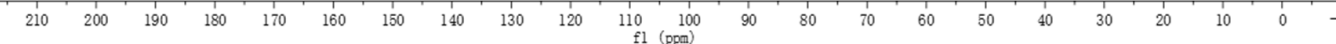



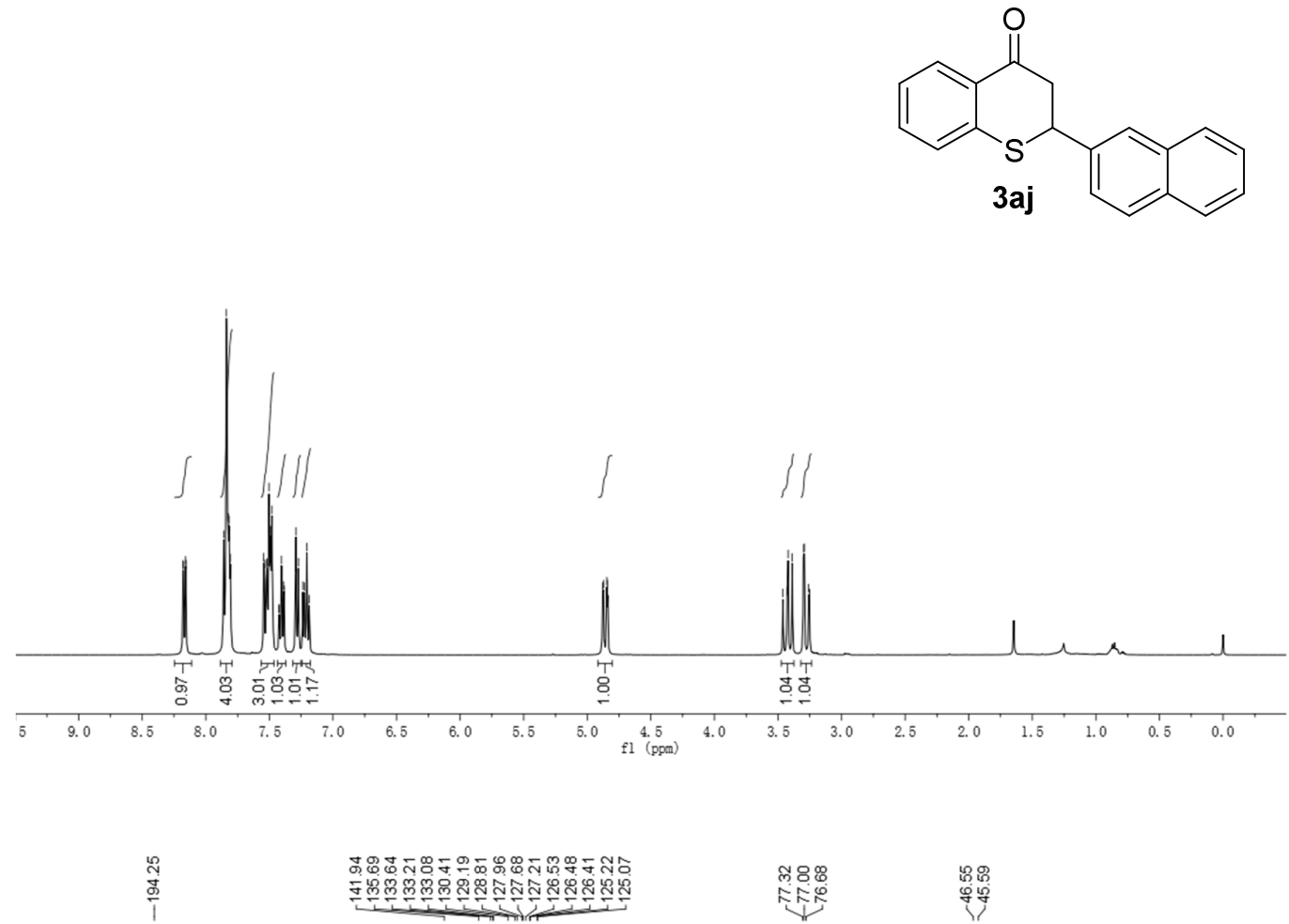

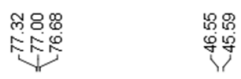
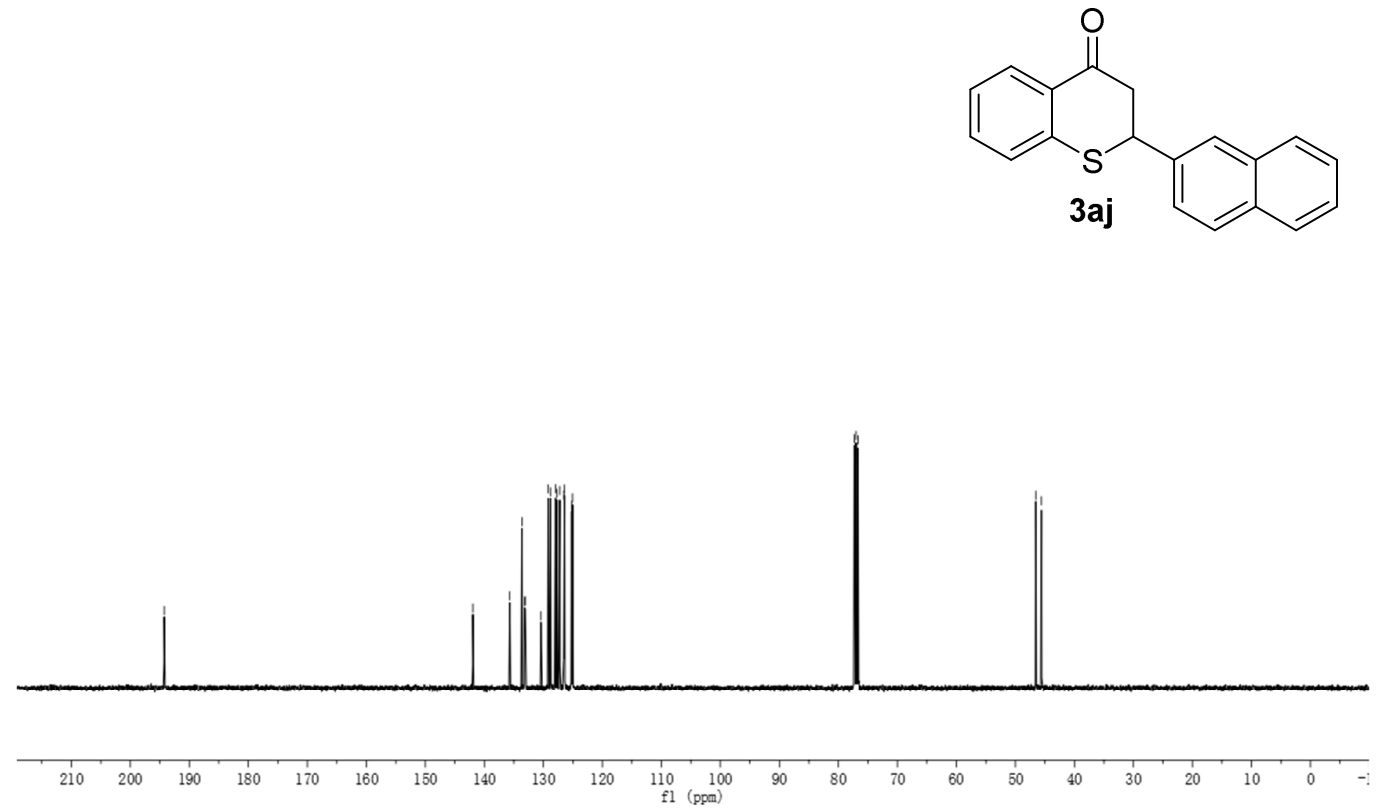


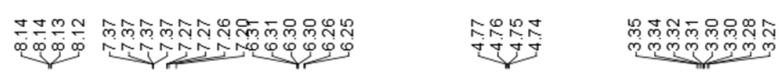
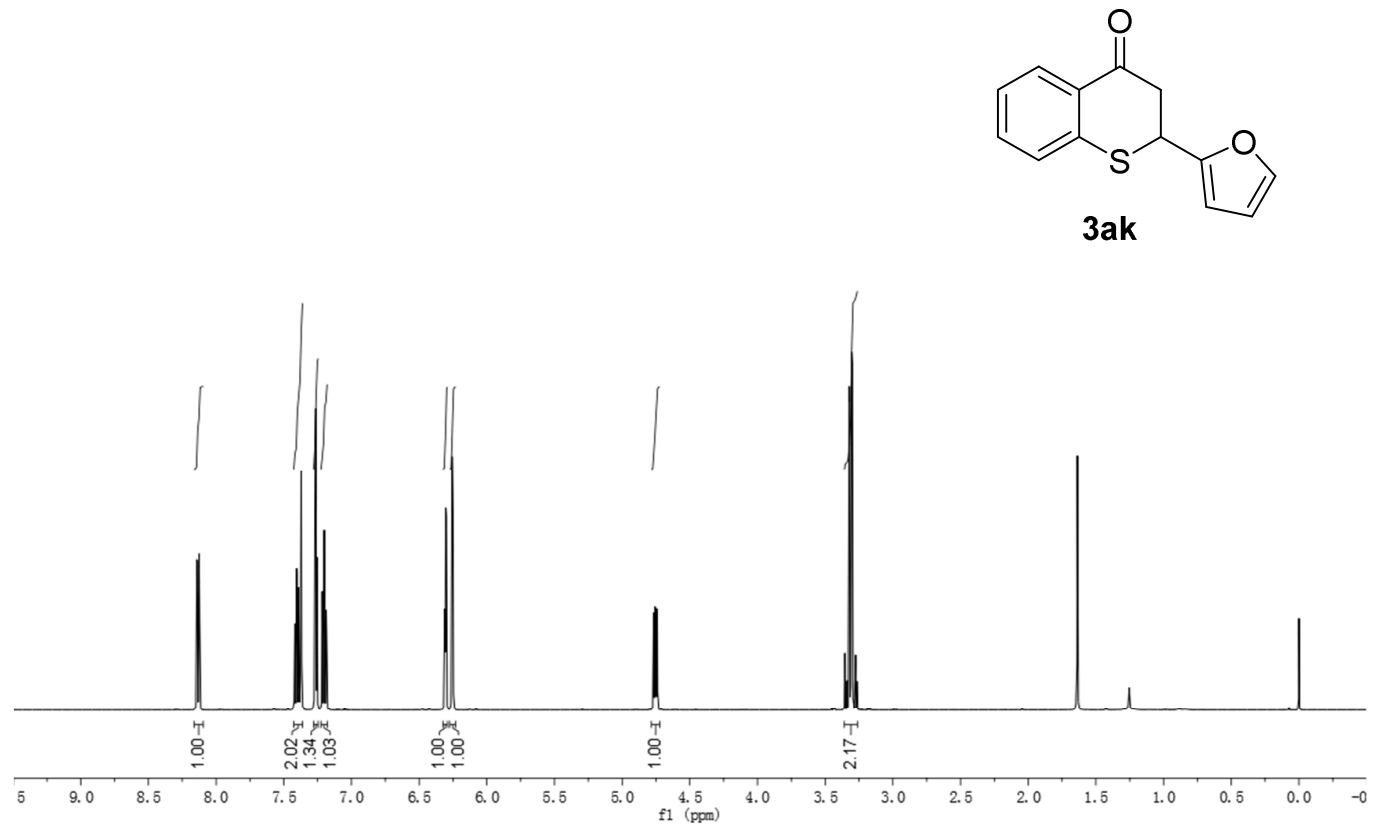

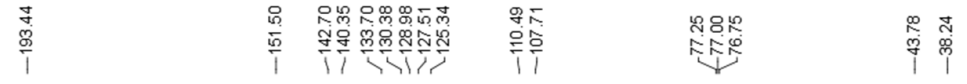

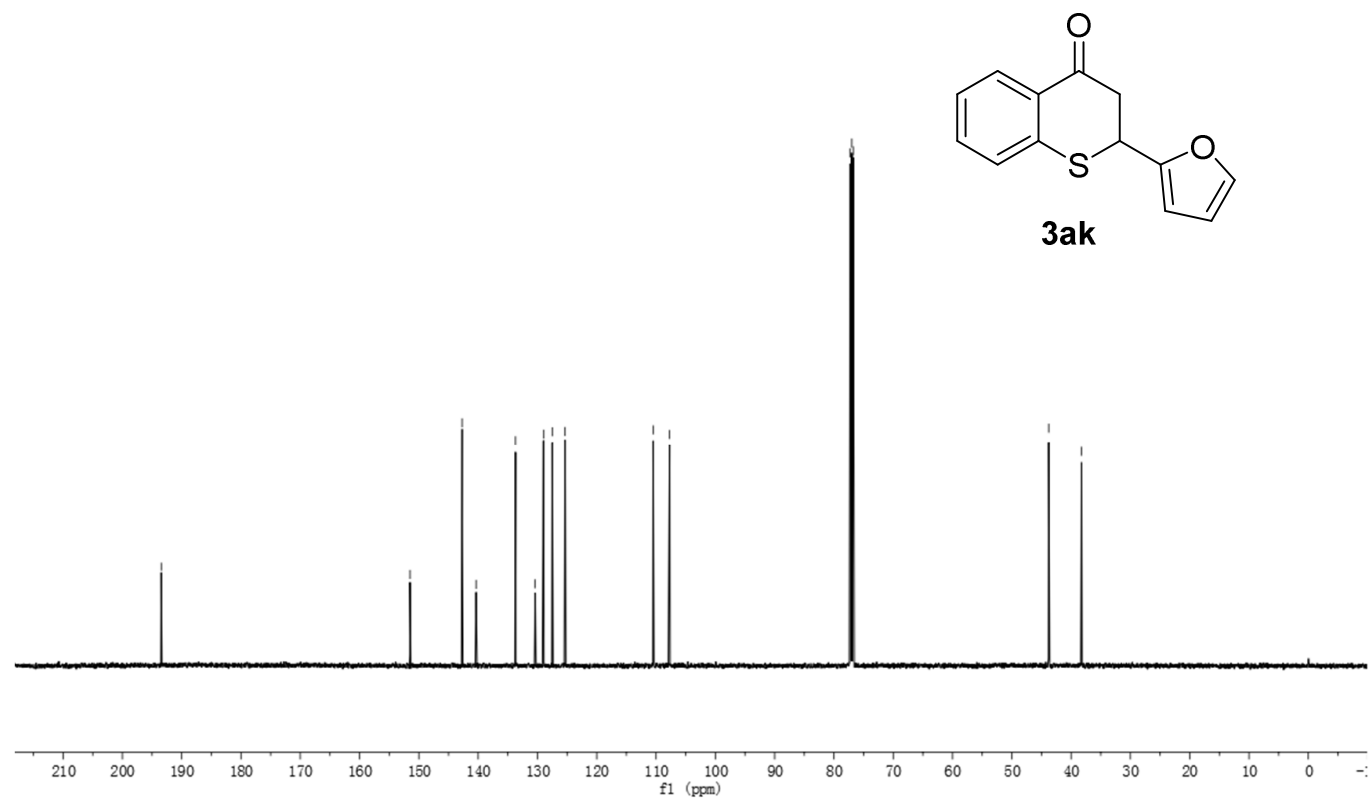




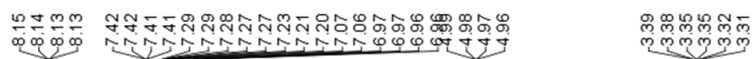

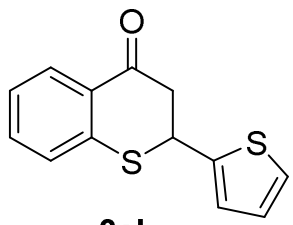

3al

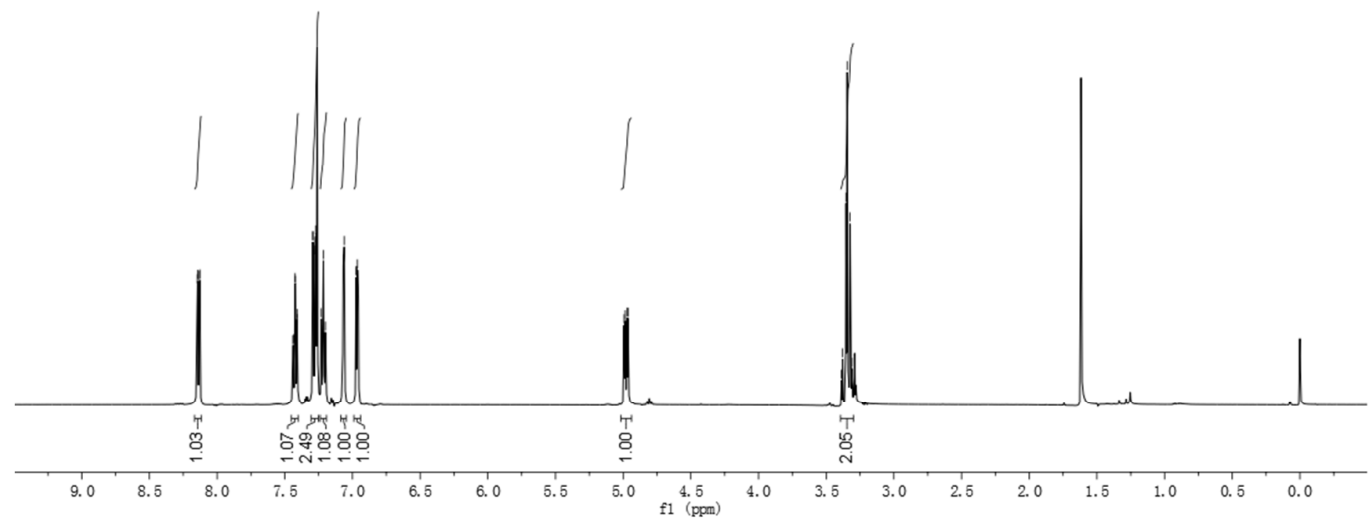

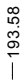

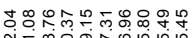

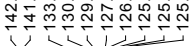

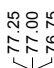

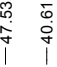

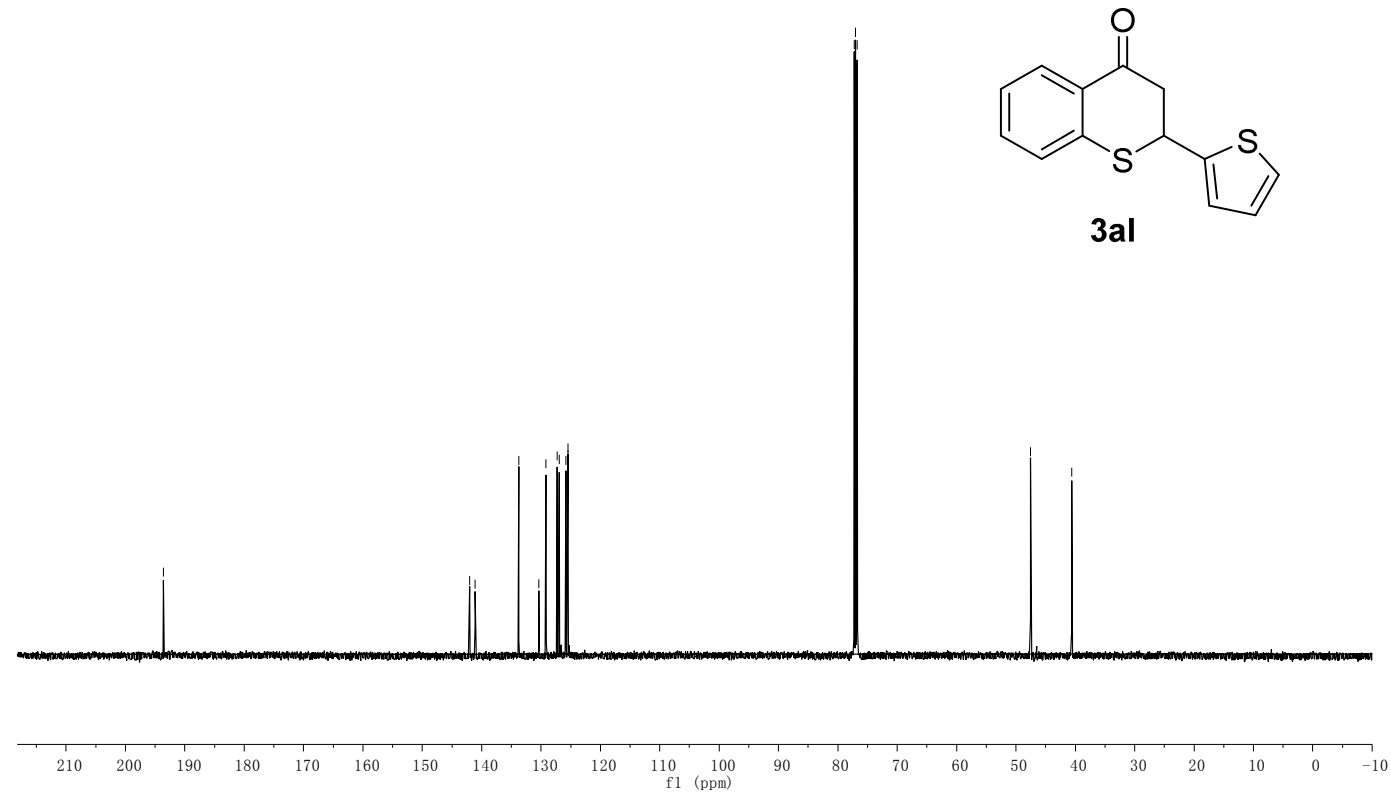



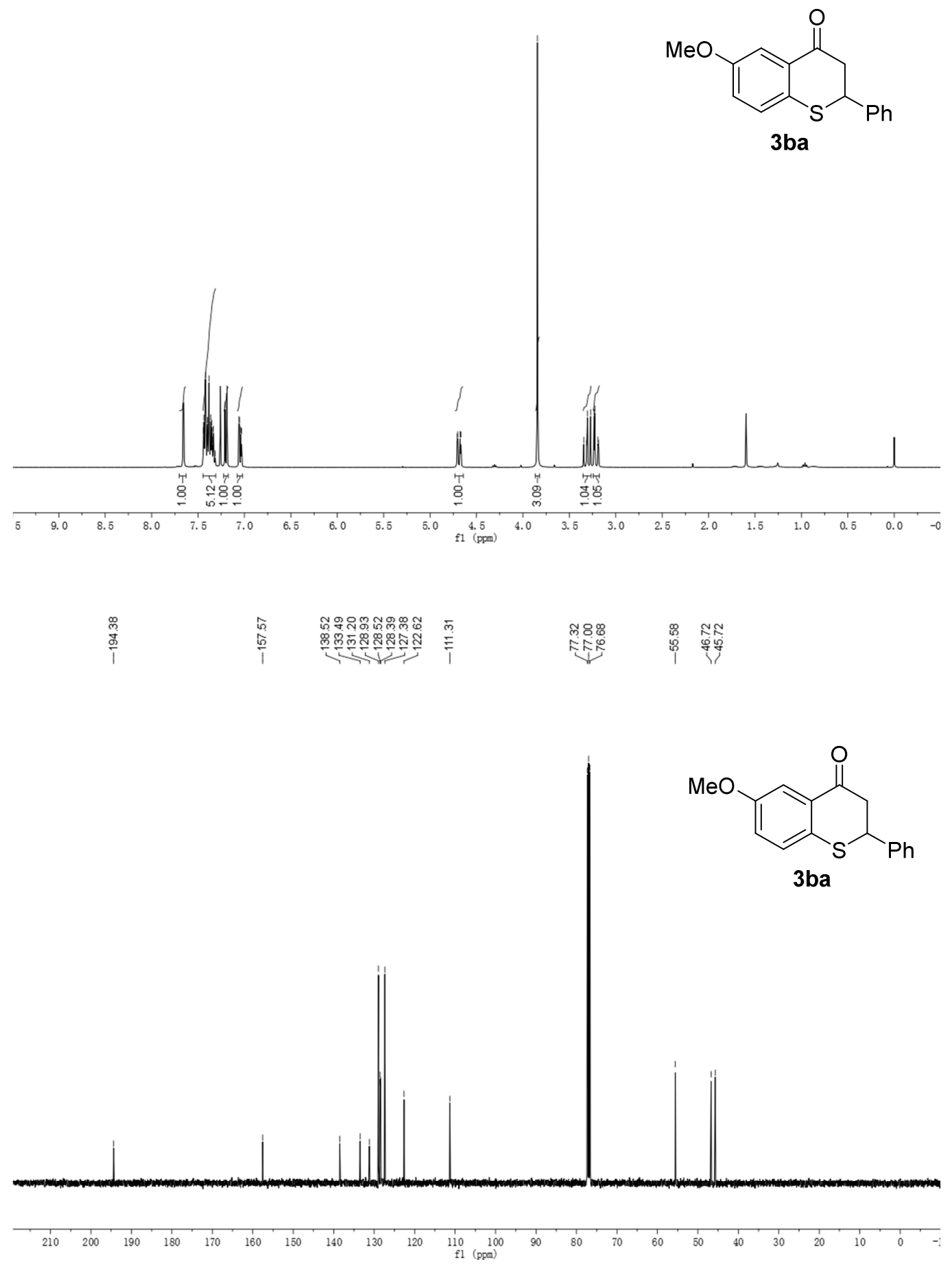

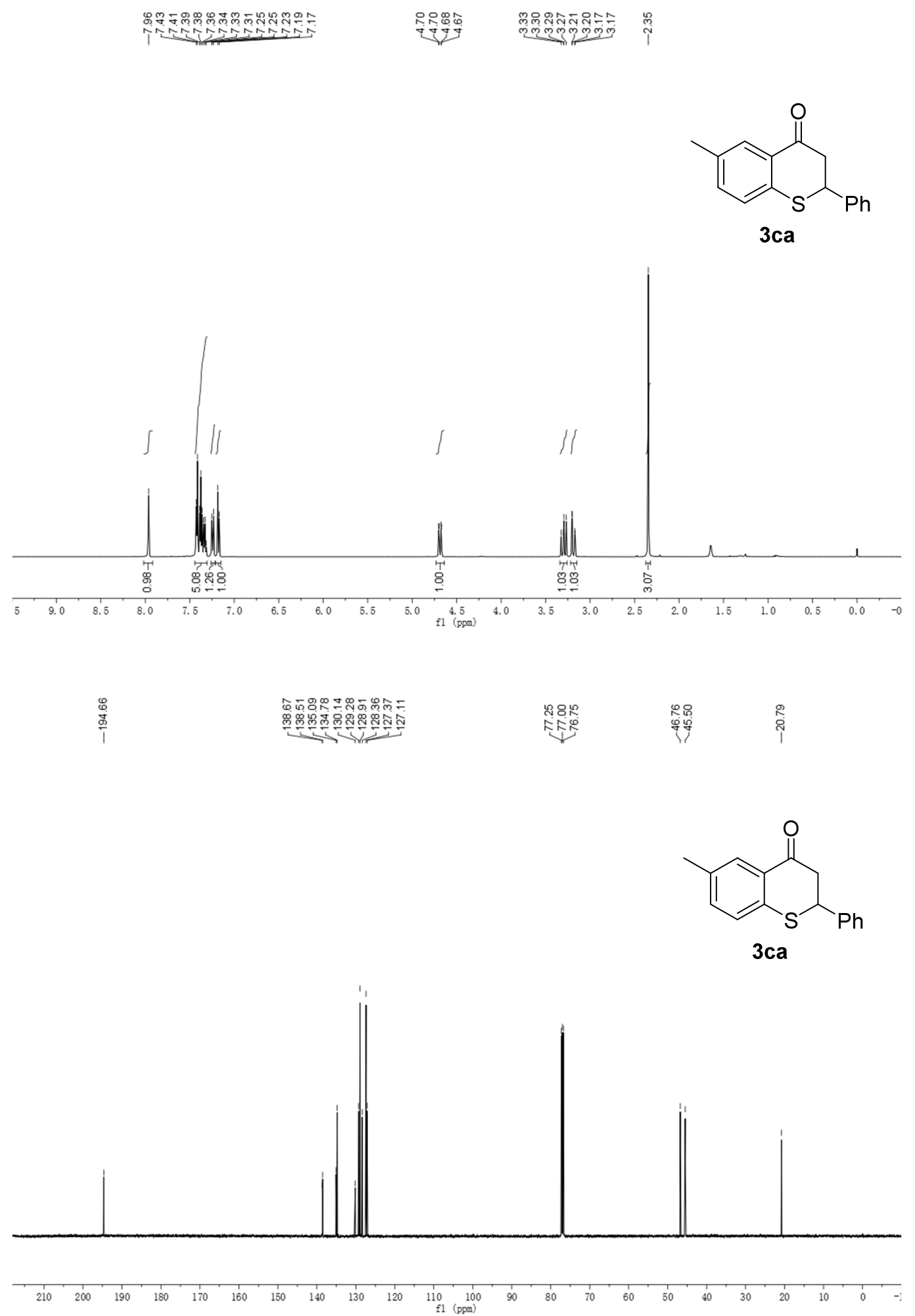

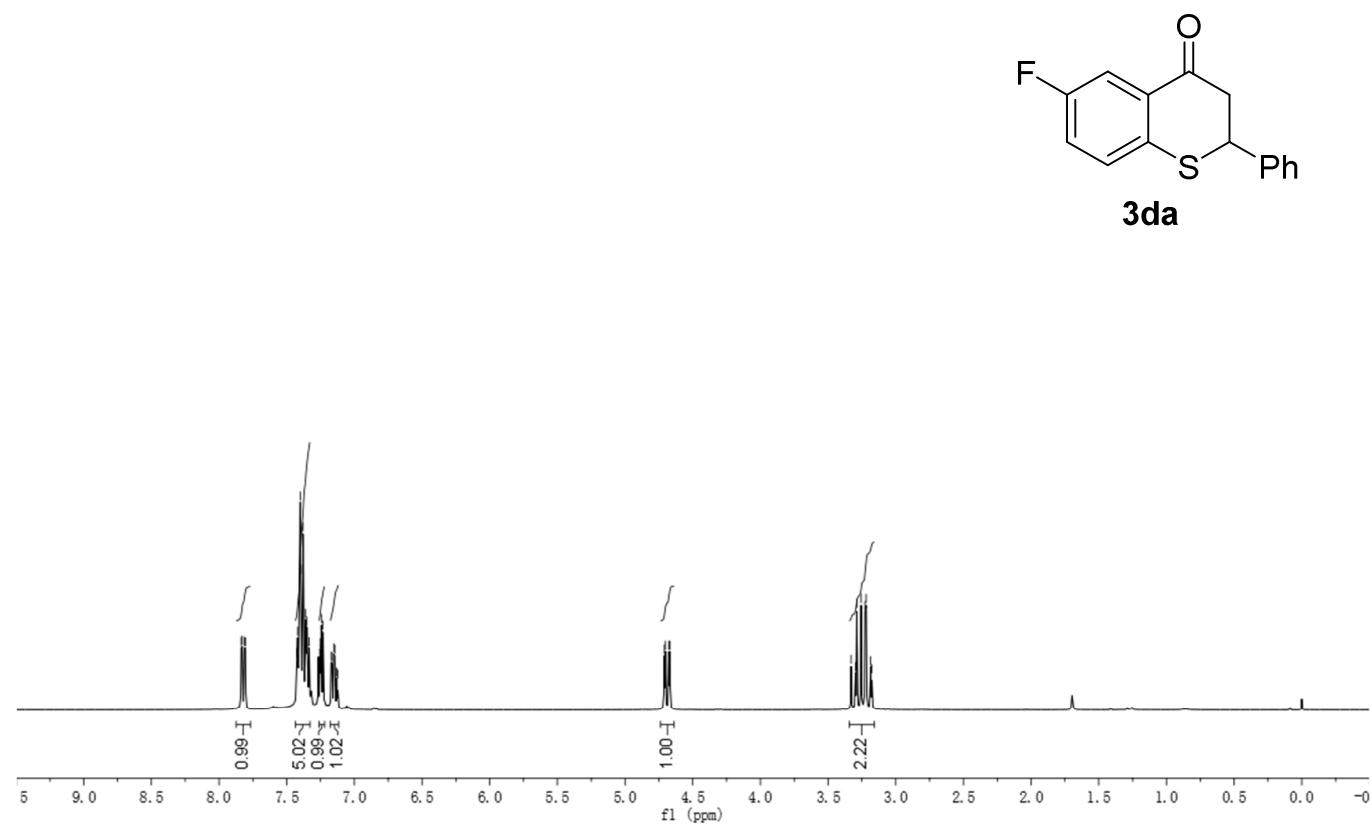

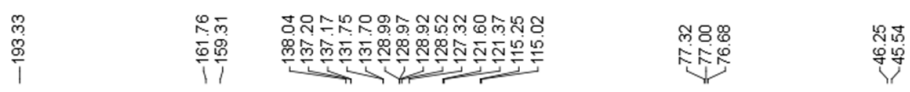
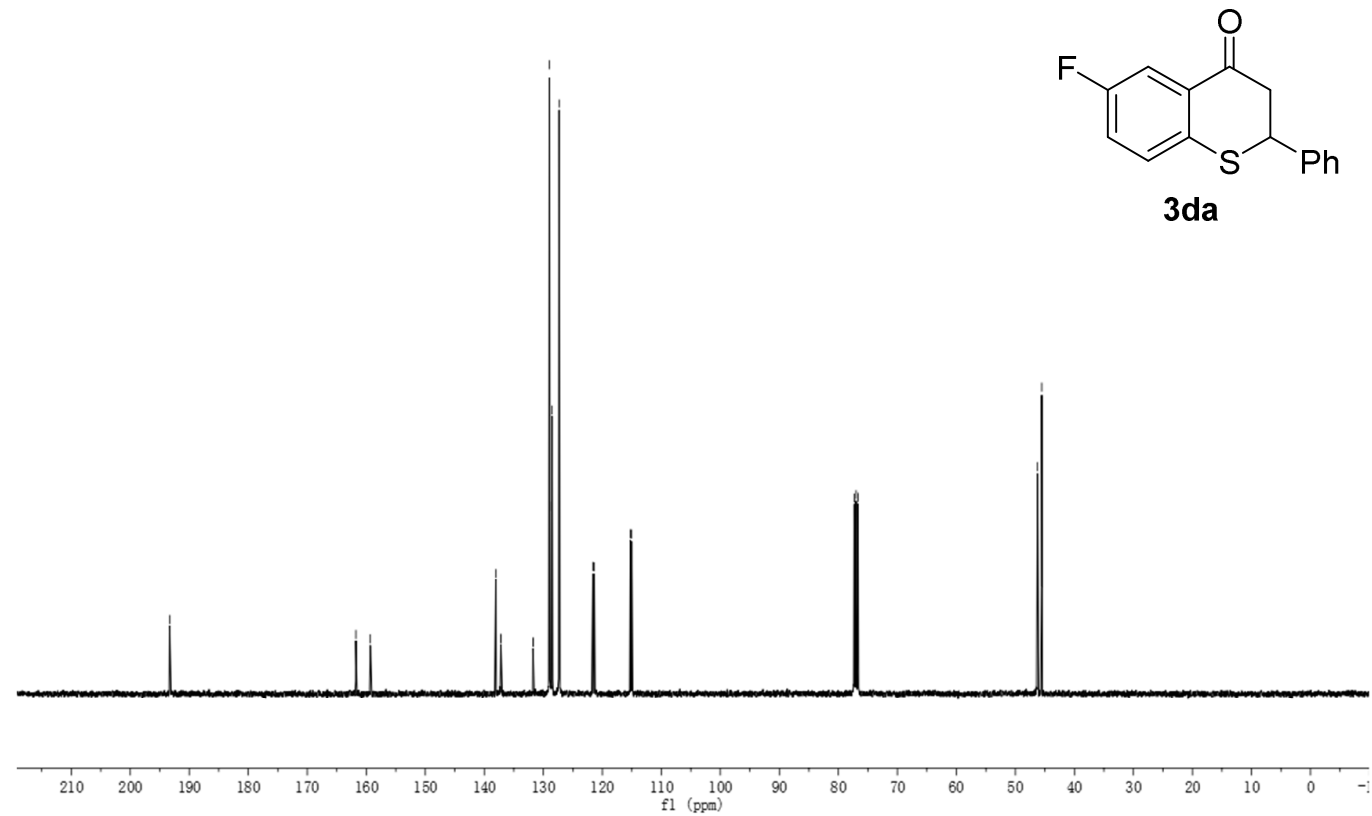

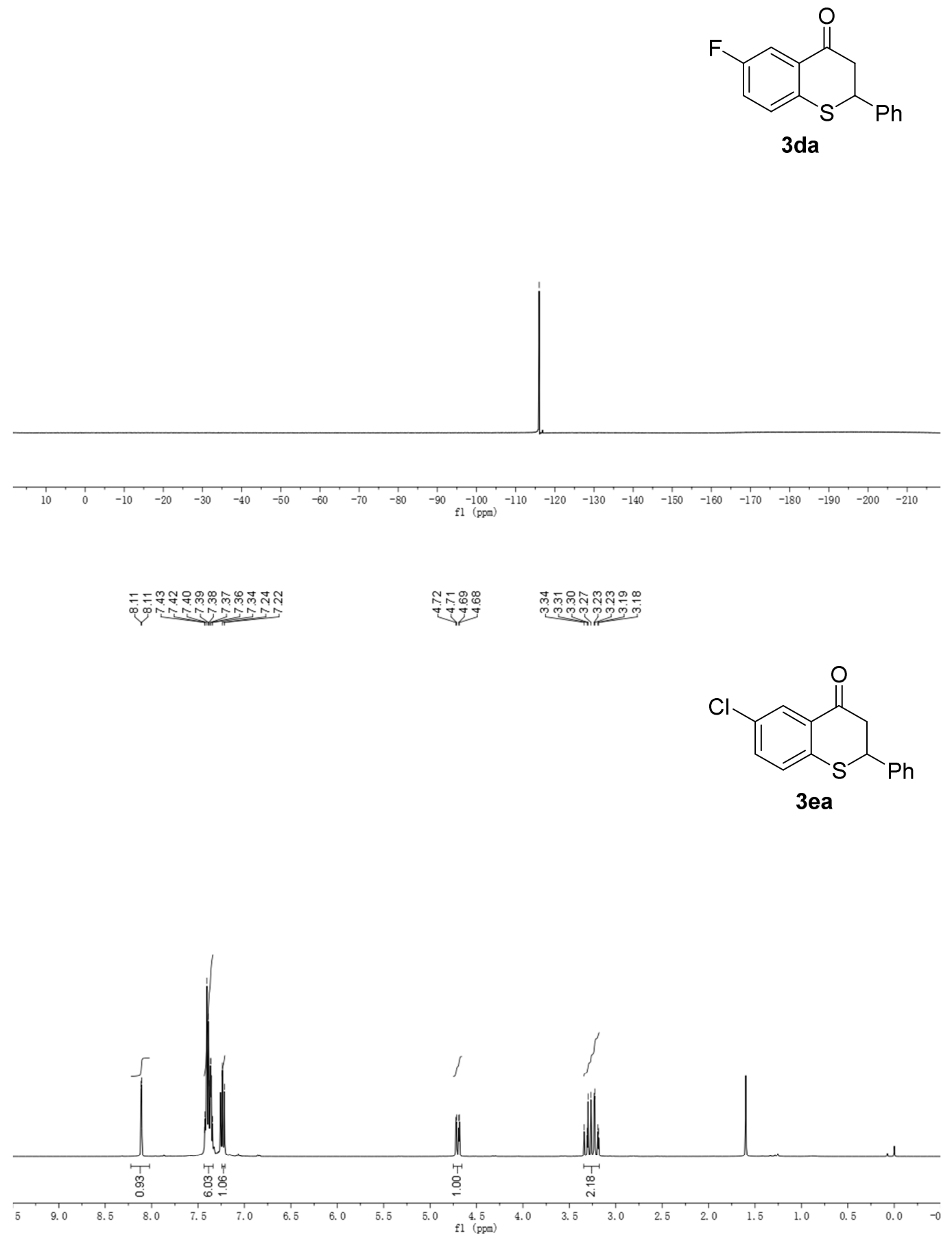


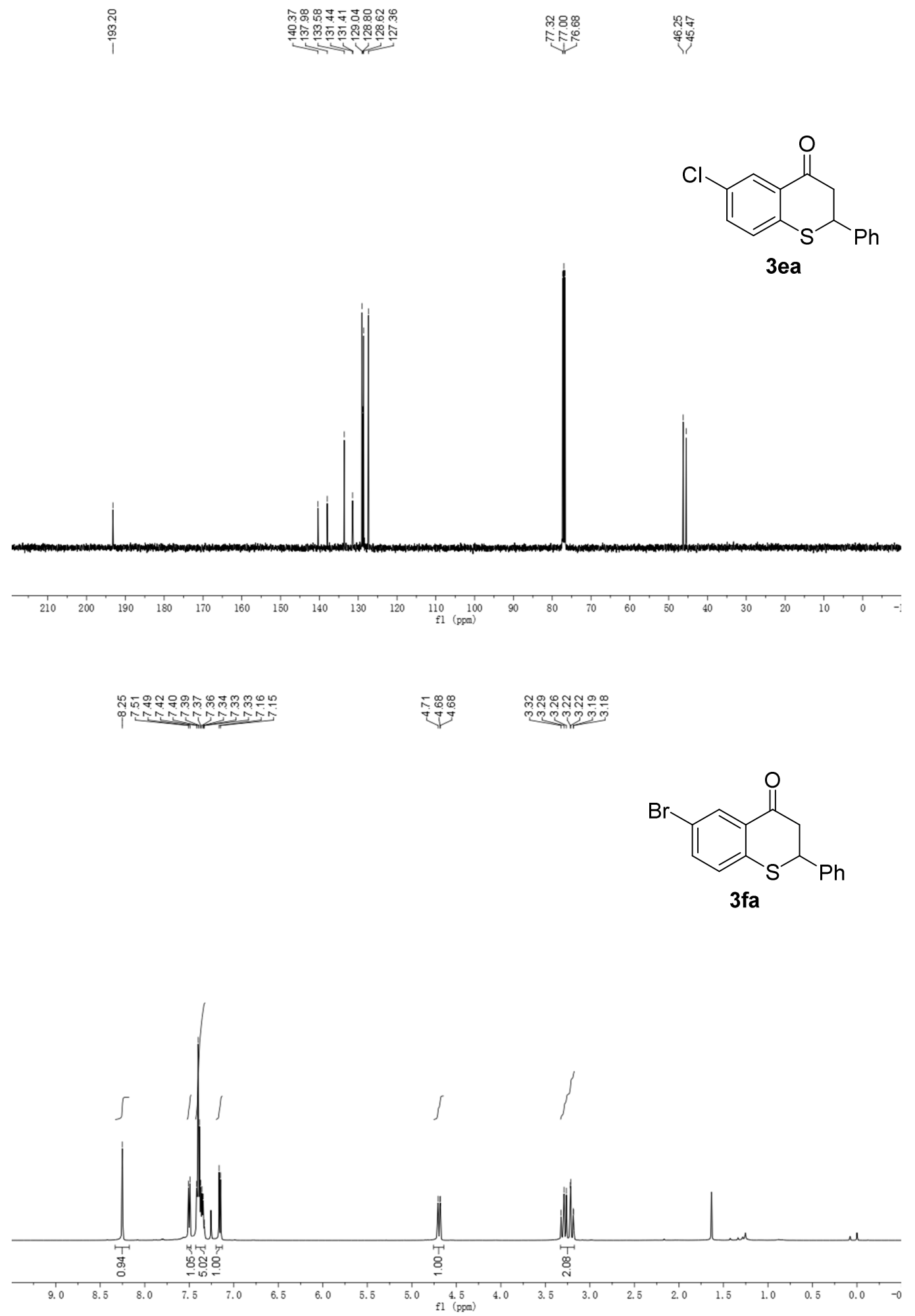




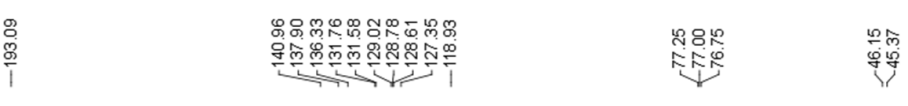
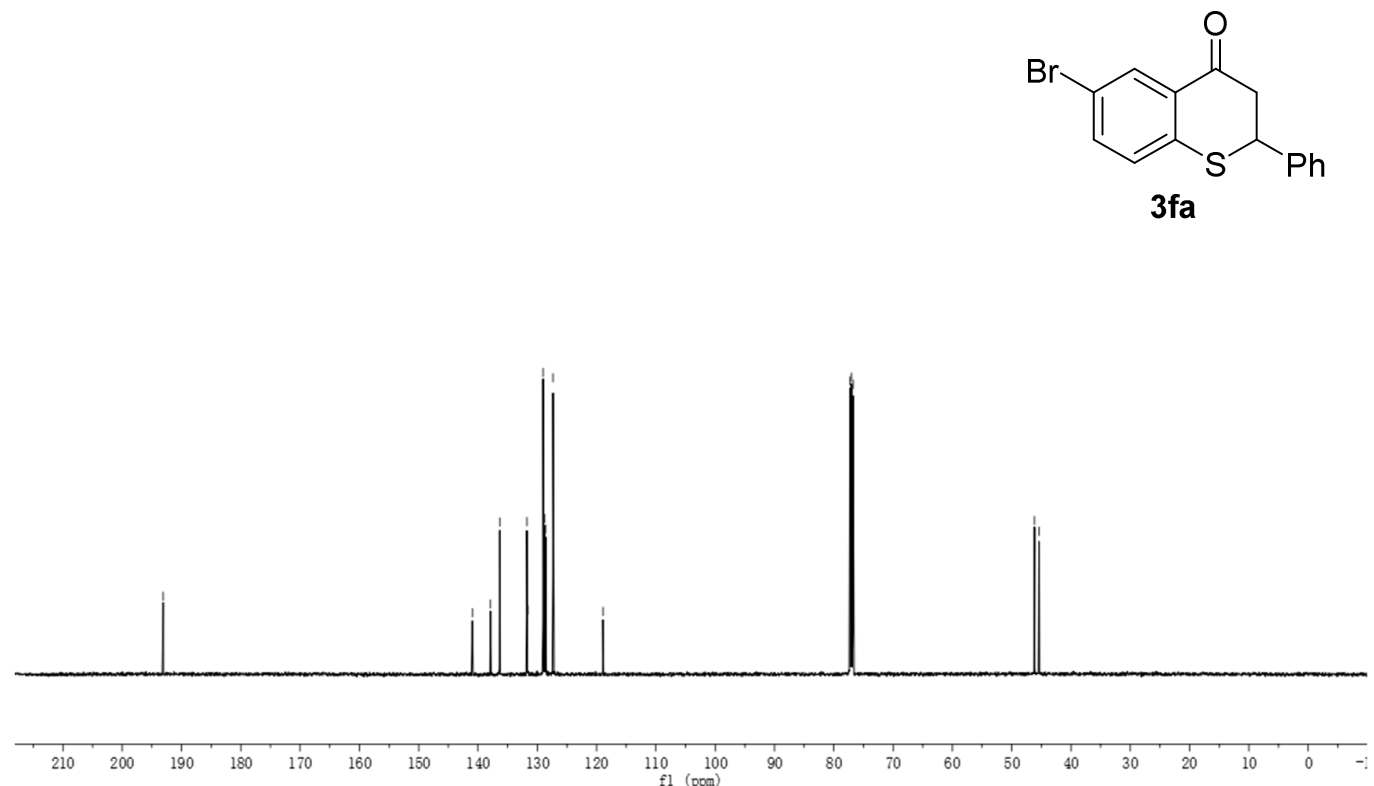

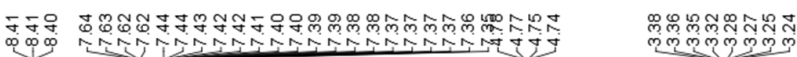
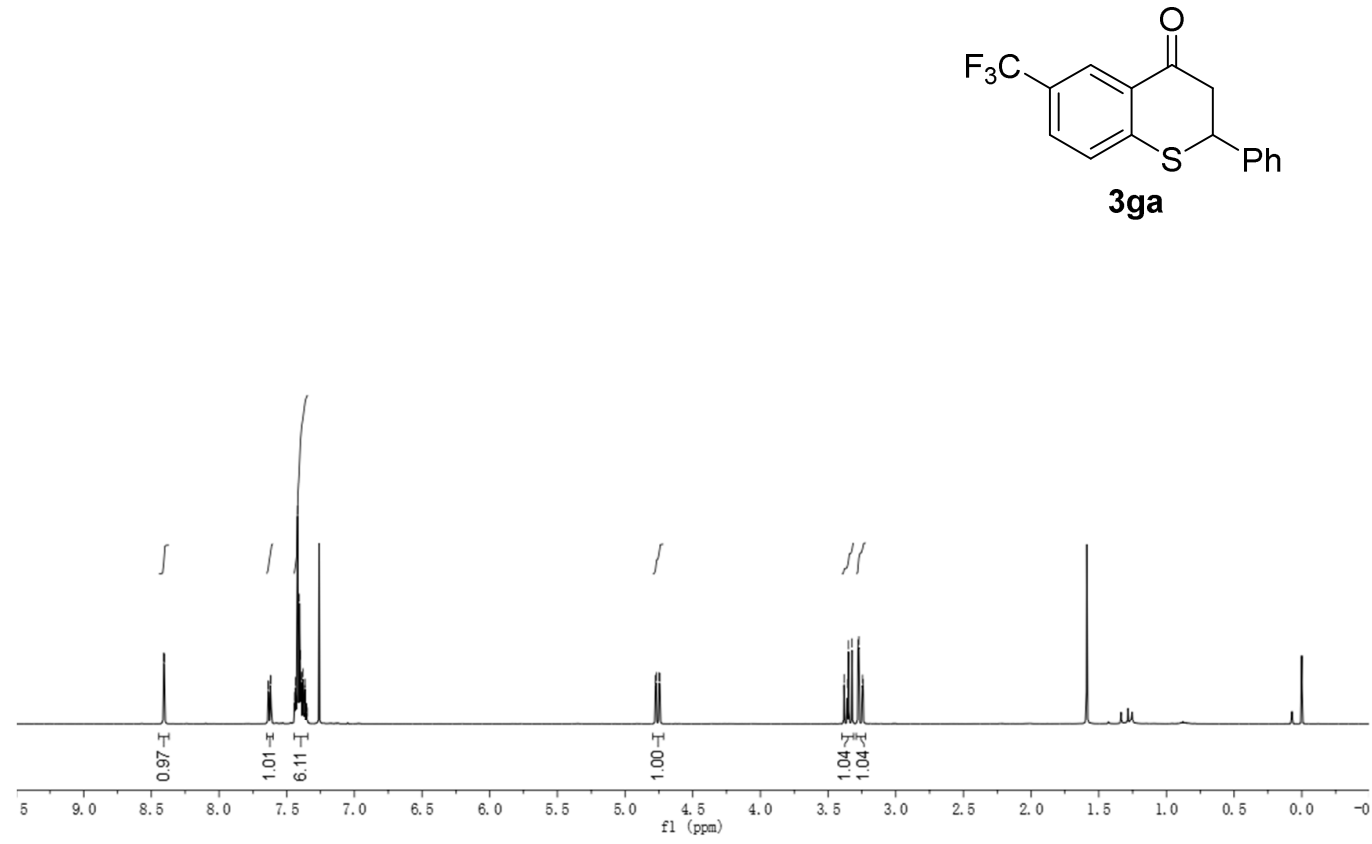

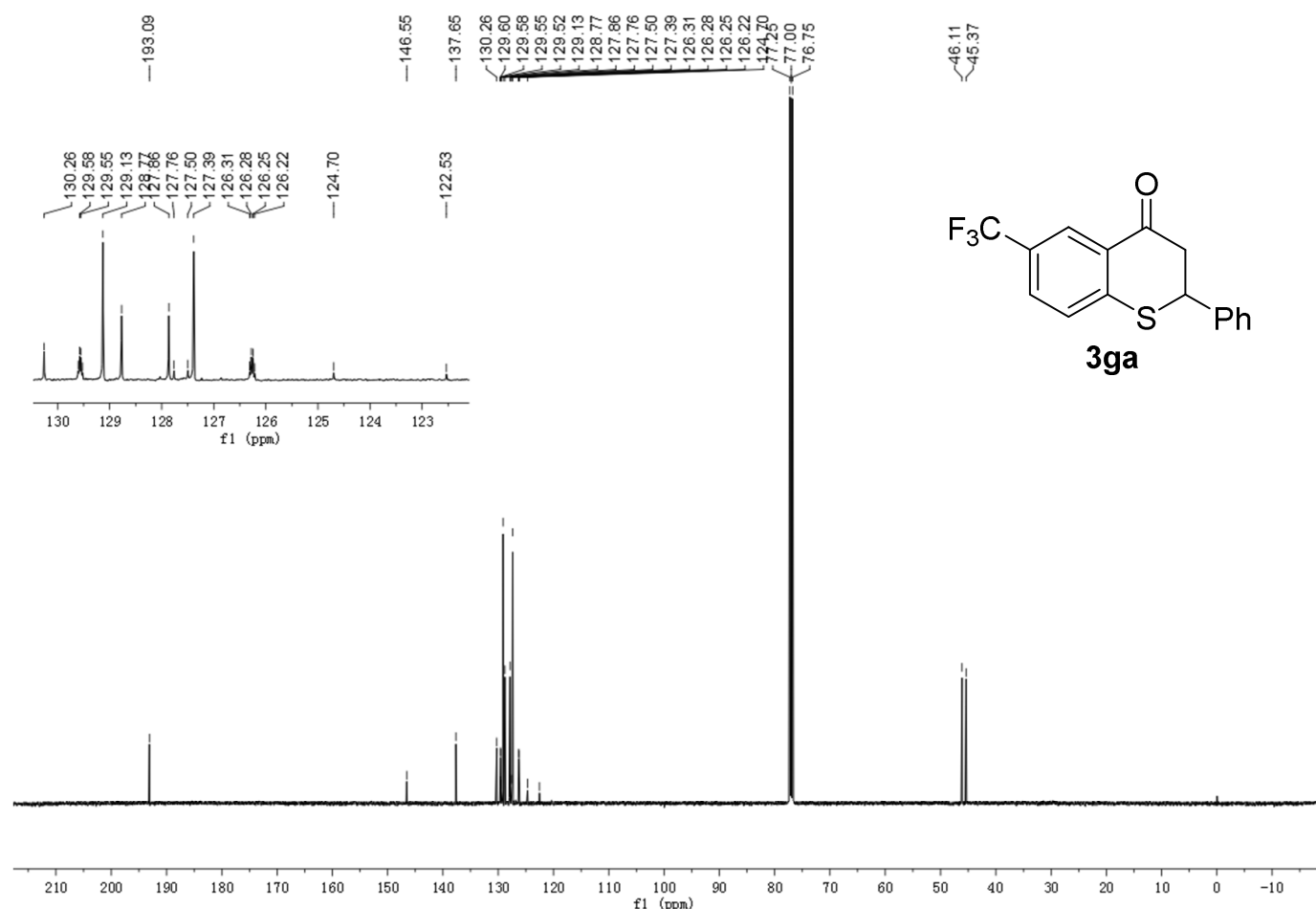

$\stackrel{\infty}{\infty}$

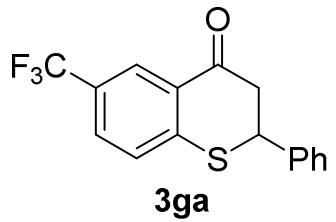

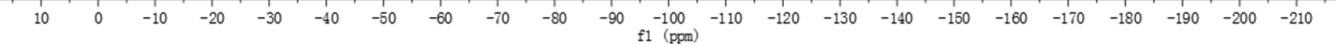




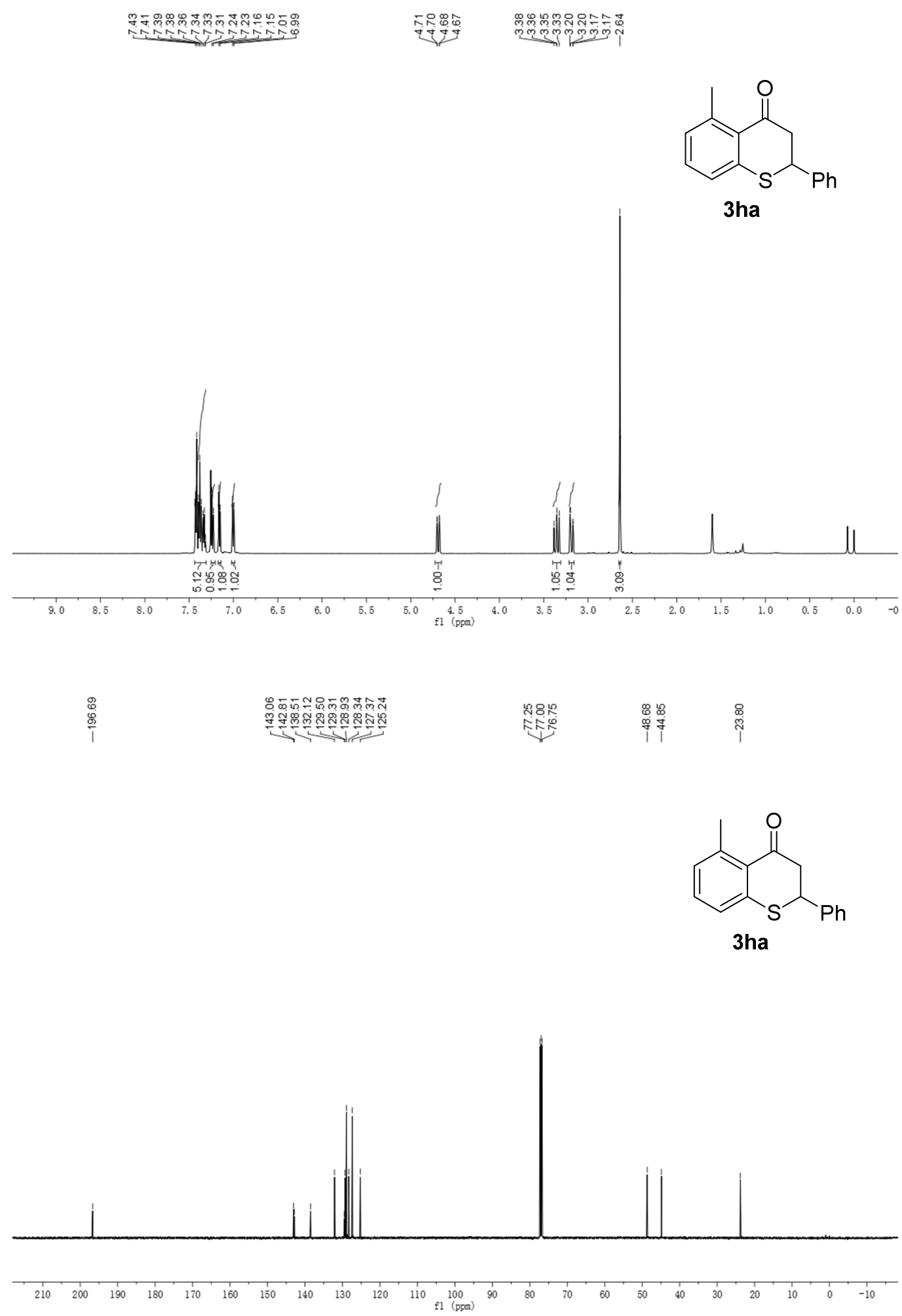




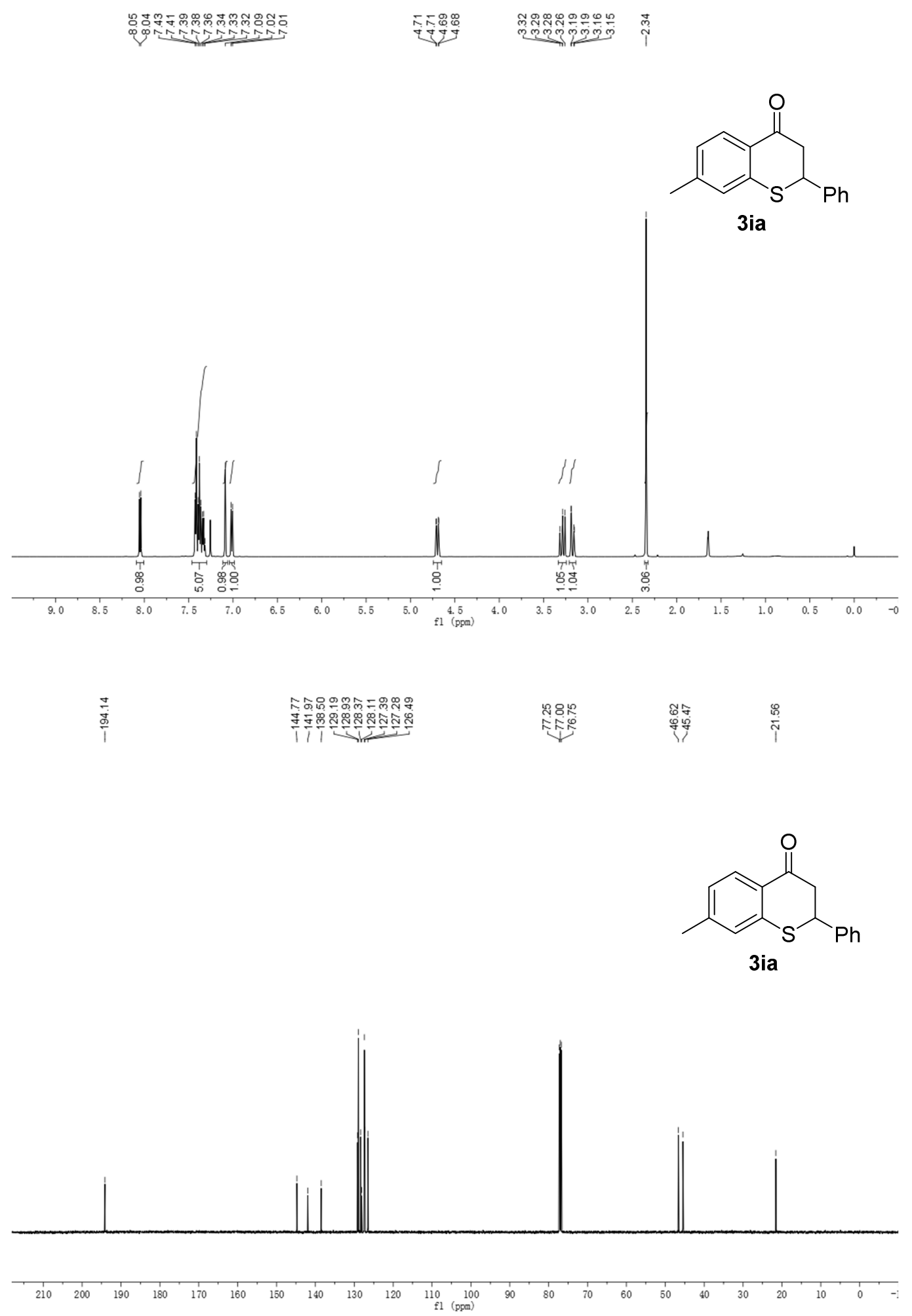



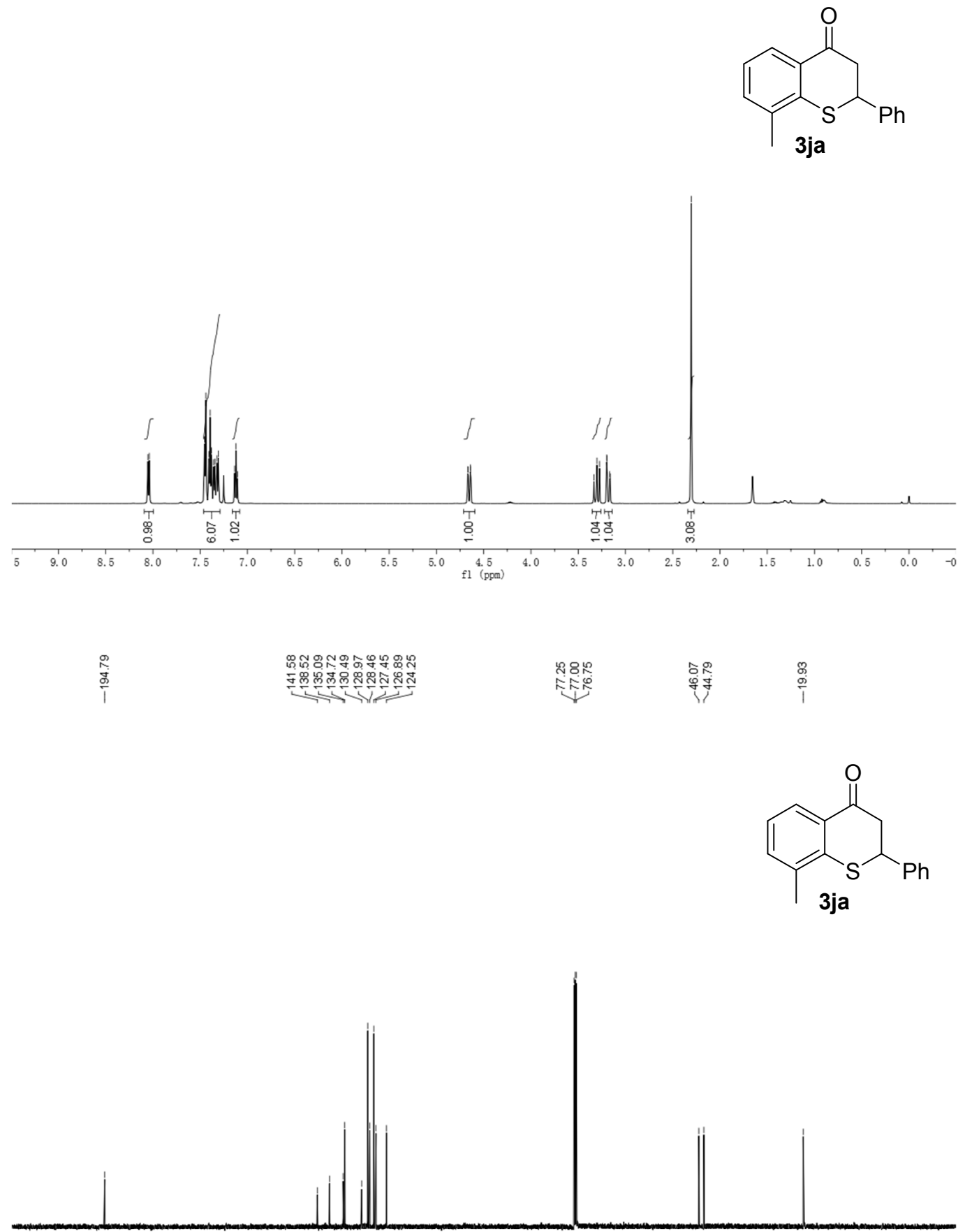

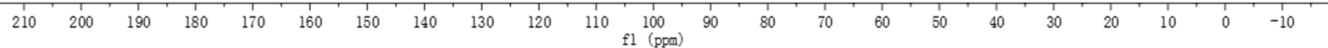



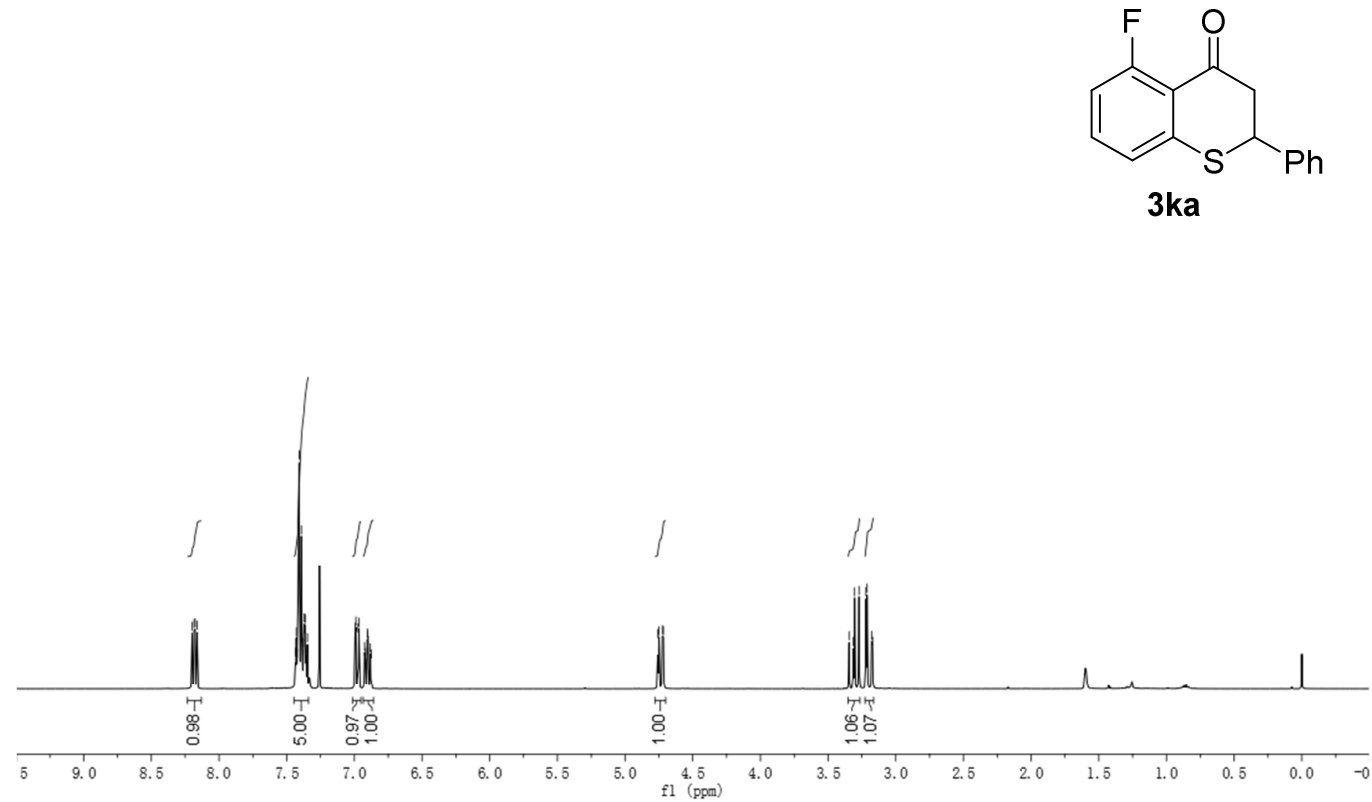

\begin{tabular}{|c|c|c|c|}
\hline 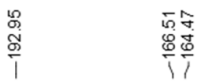 & 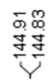 & 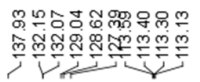 & 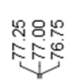 \\
\hline
\end{tabular}

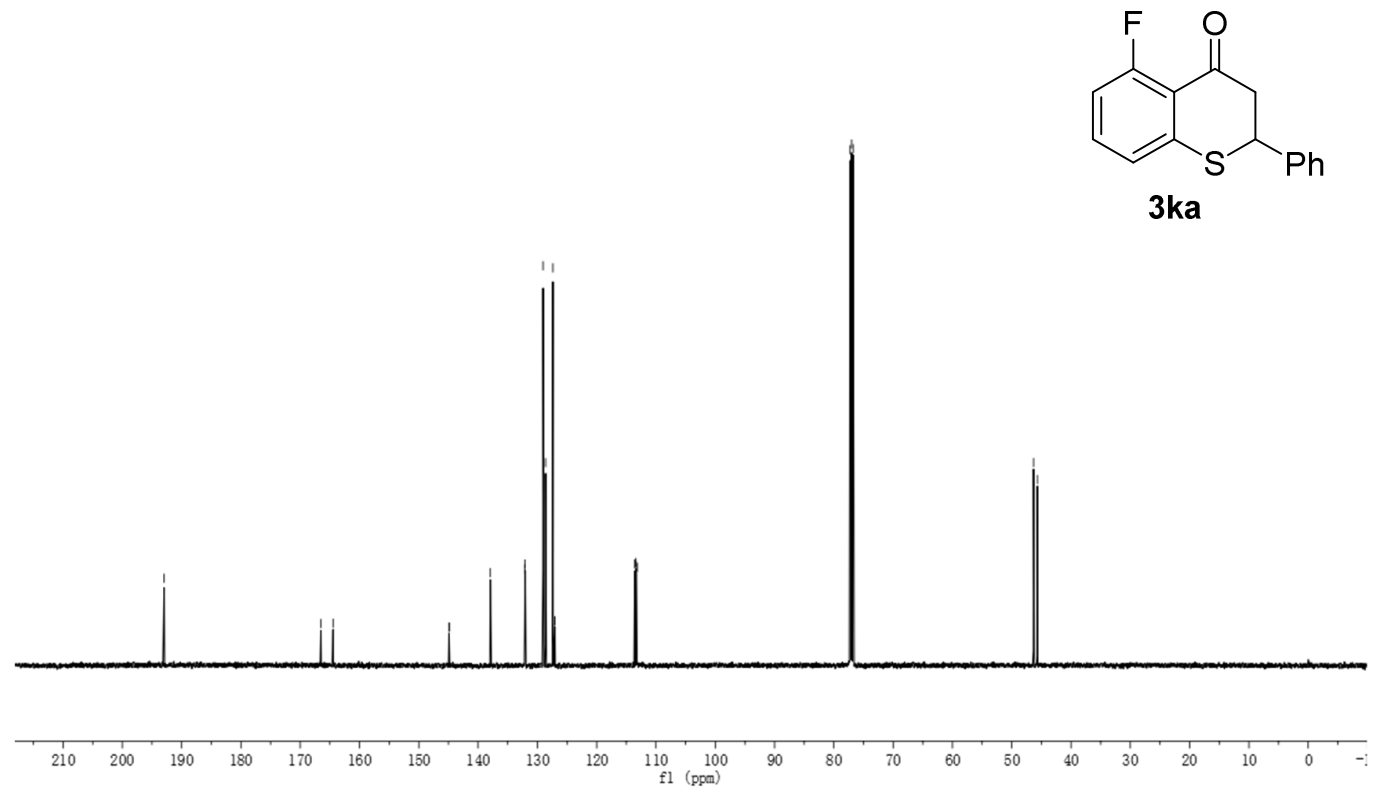



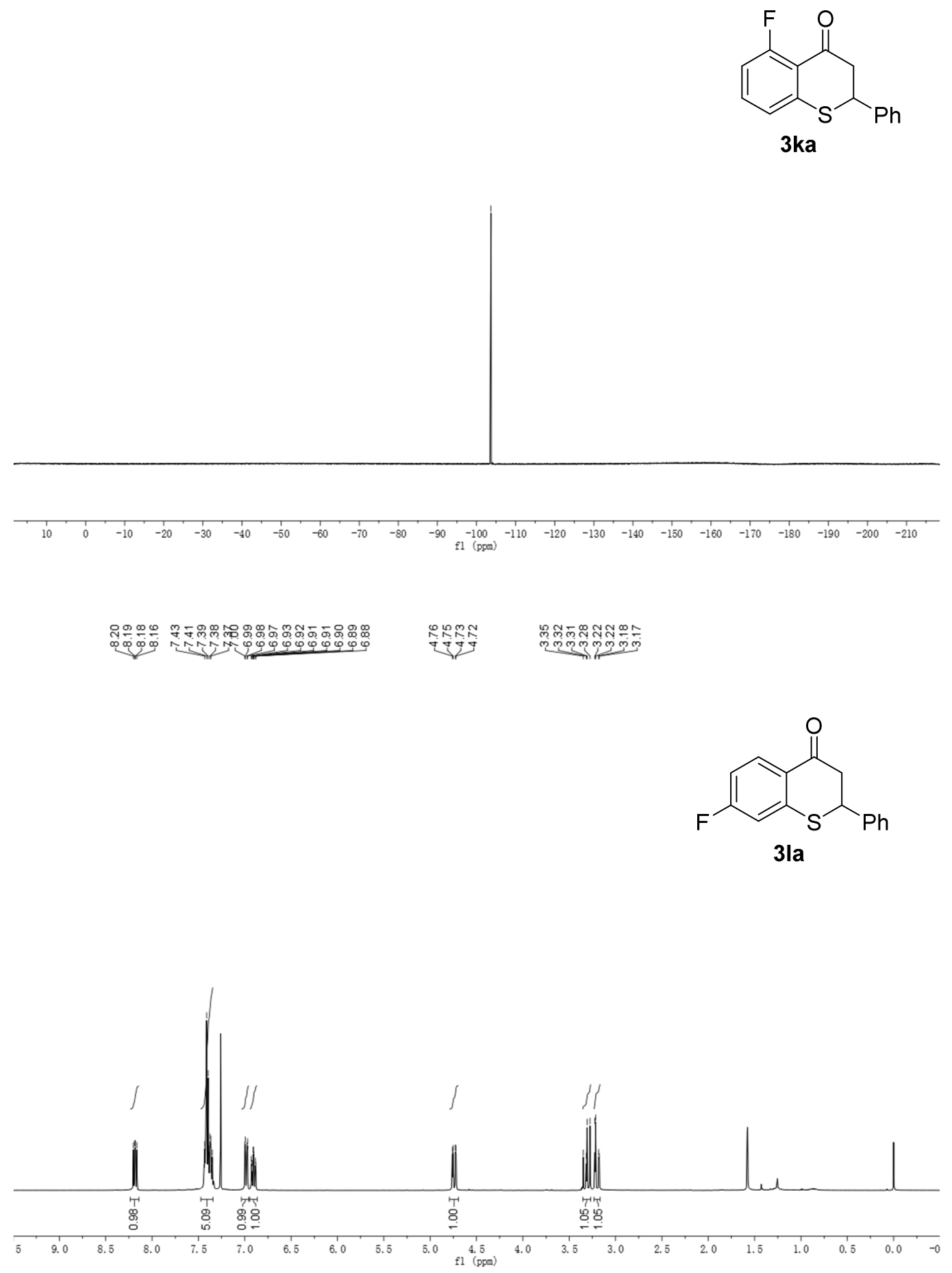

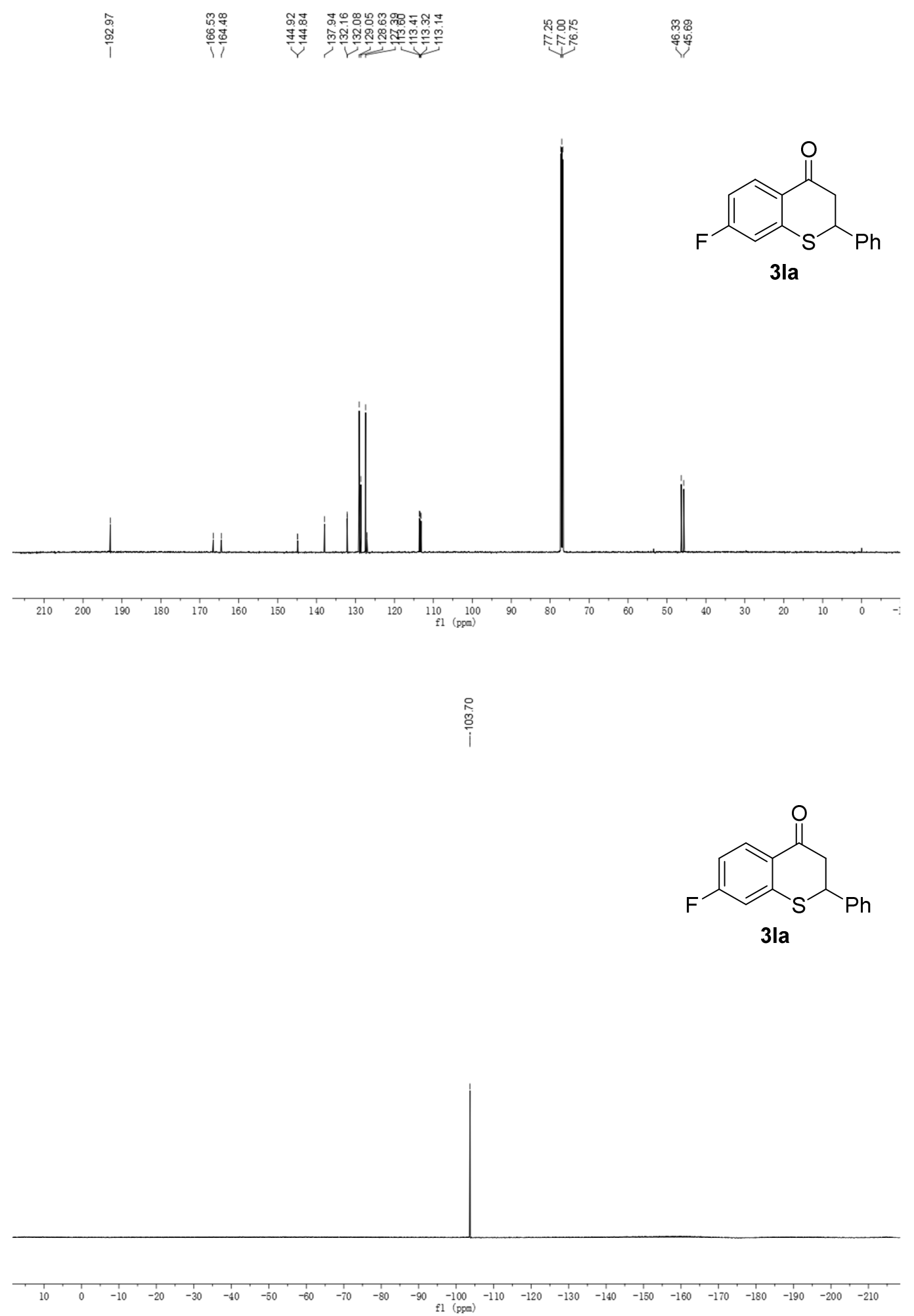

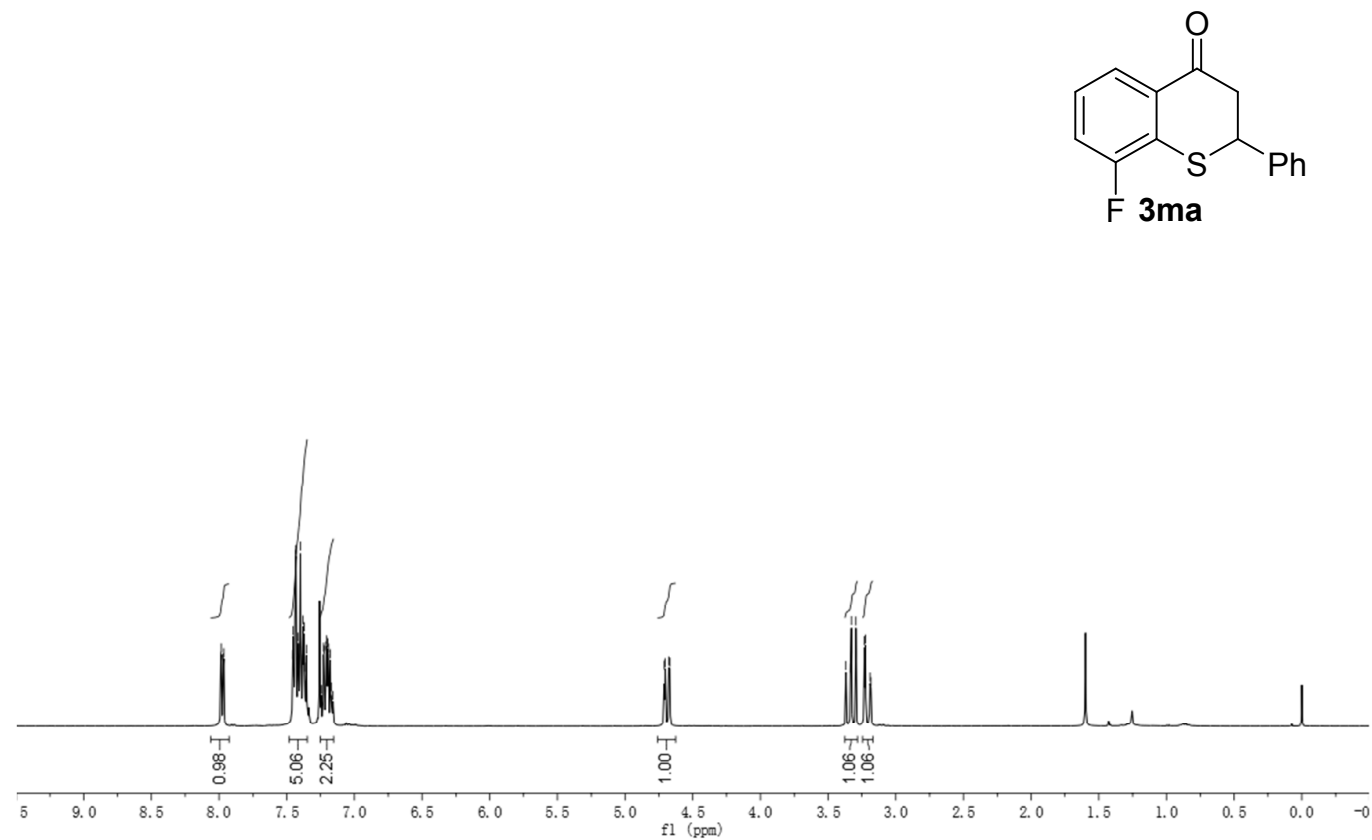

\begin{tabular}{|c|c|c|c|}
\hline ্ֻলু & 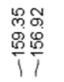 & 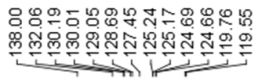 & 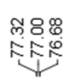 \\
\hline
\end{tabular}

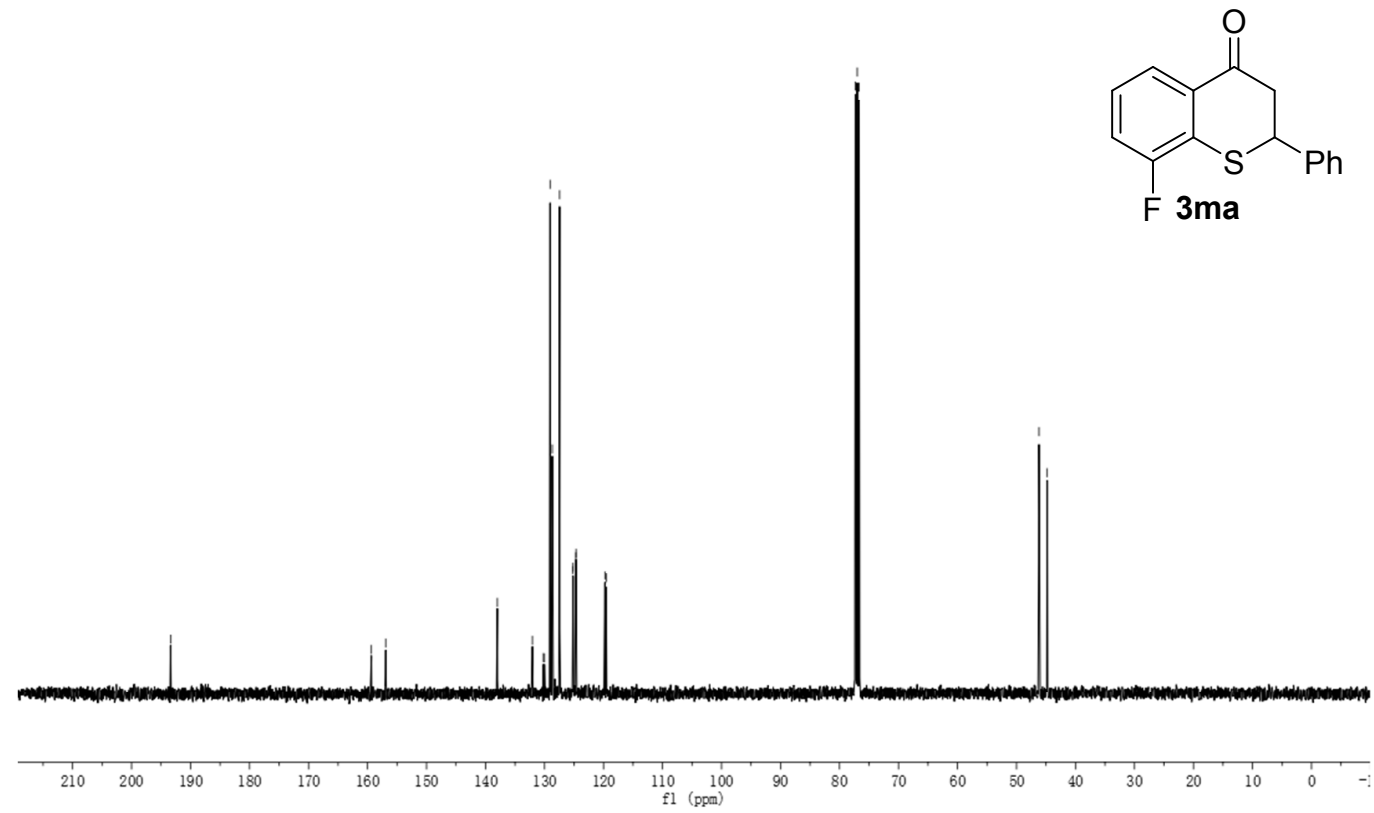



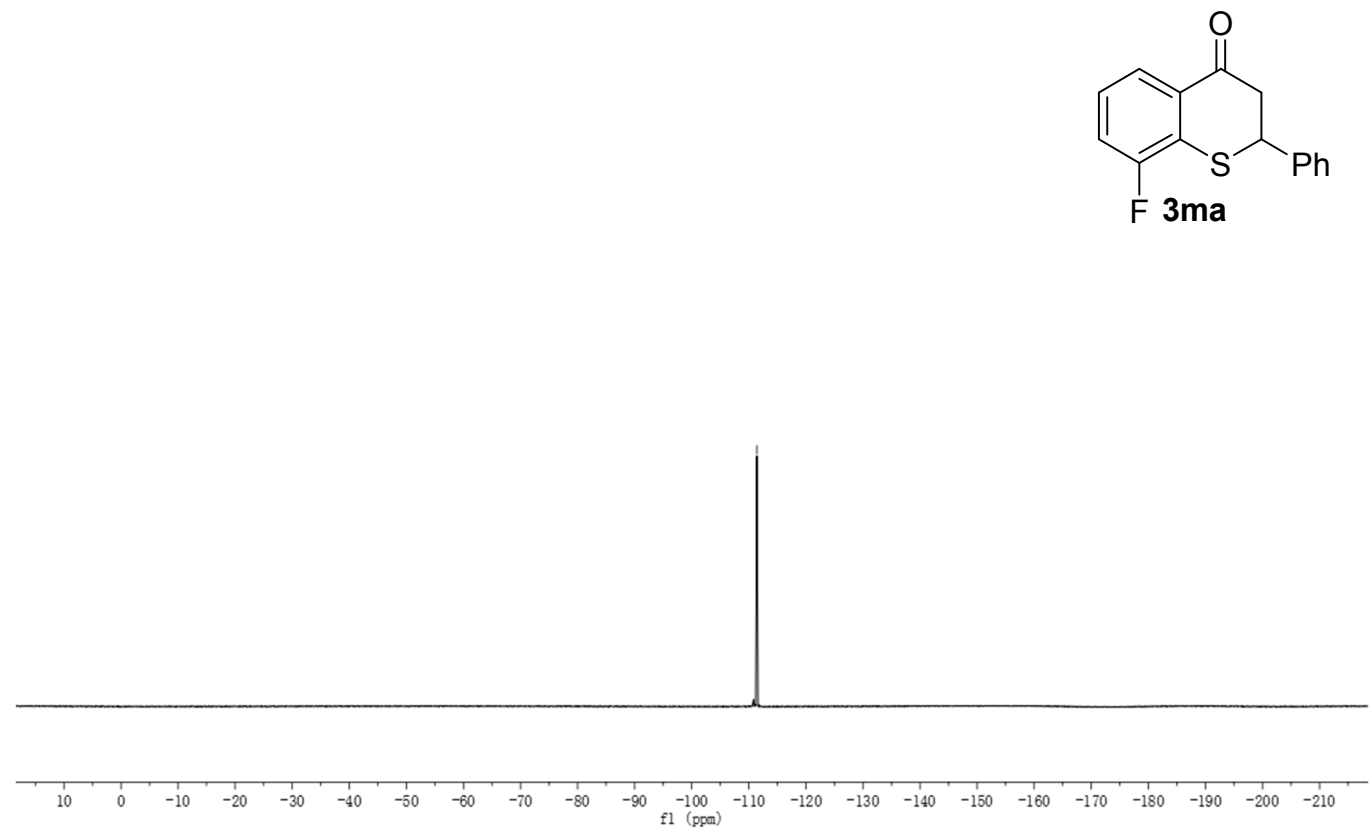

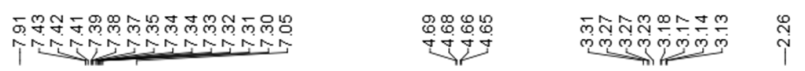

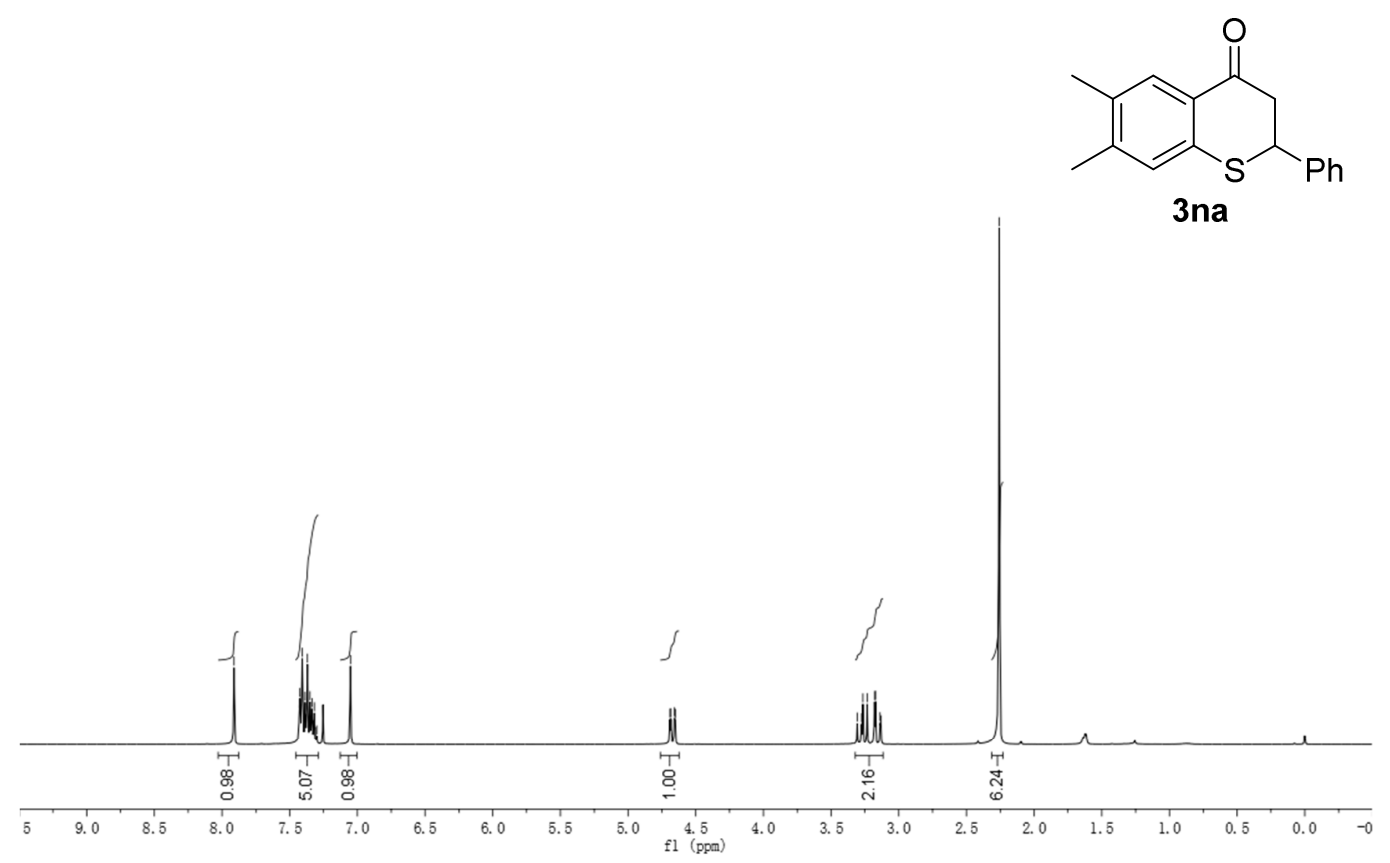



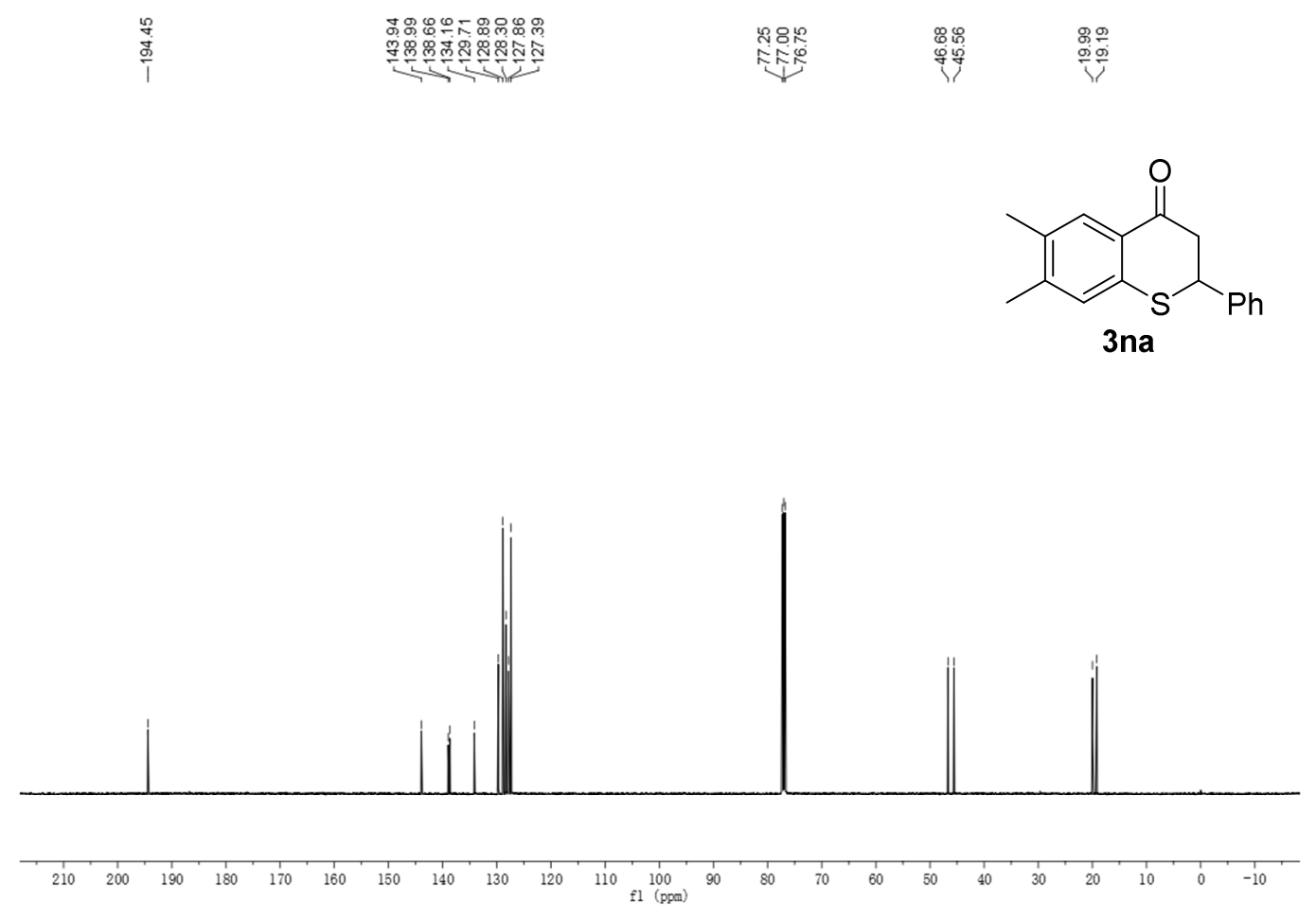

身
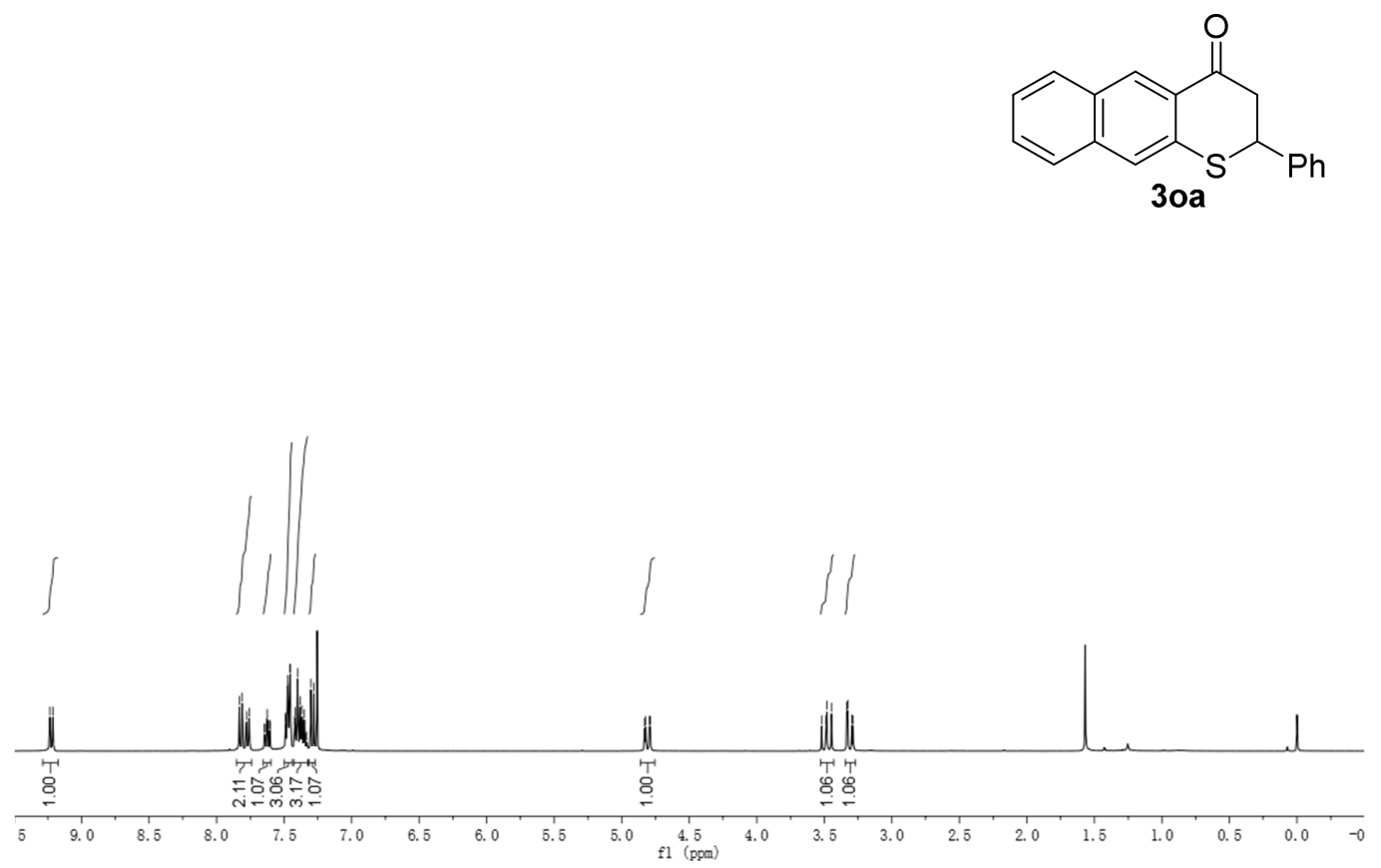


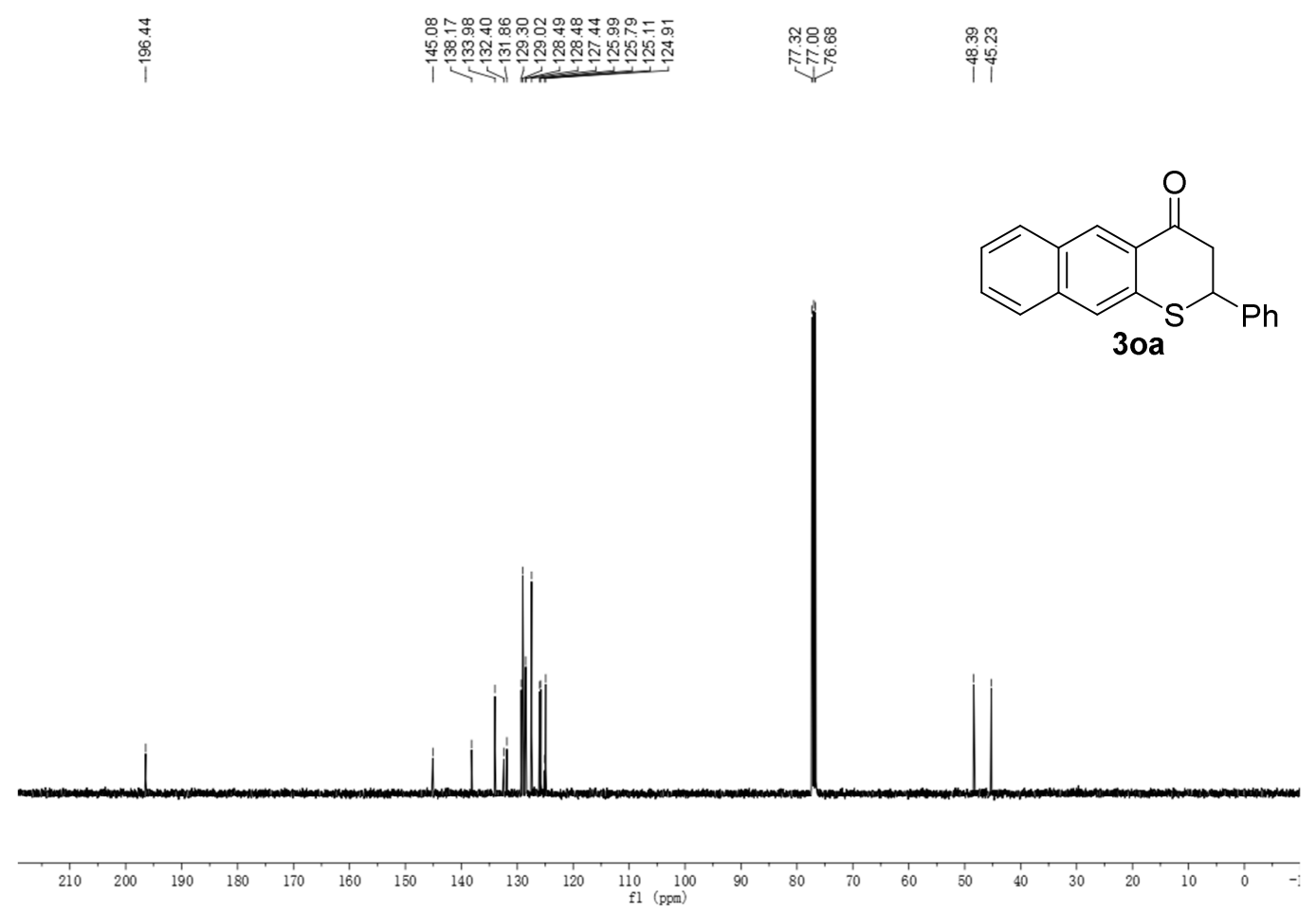

\section{HPLC Traces}

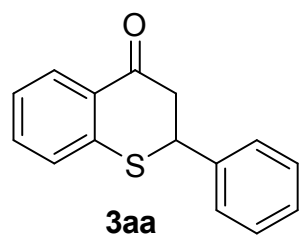

HPLC trace for racemic-3aa

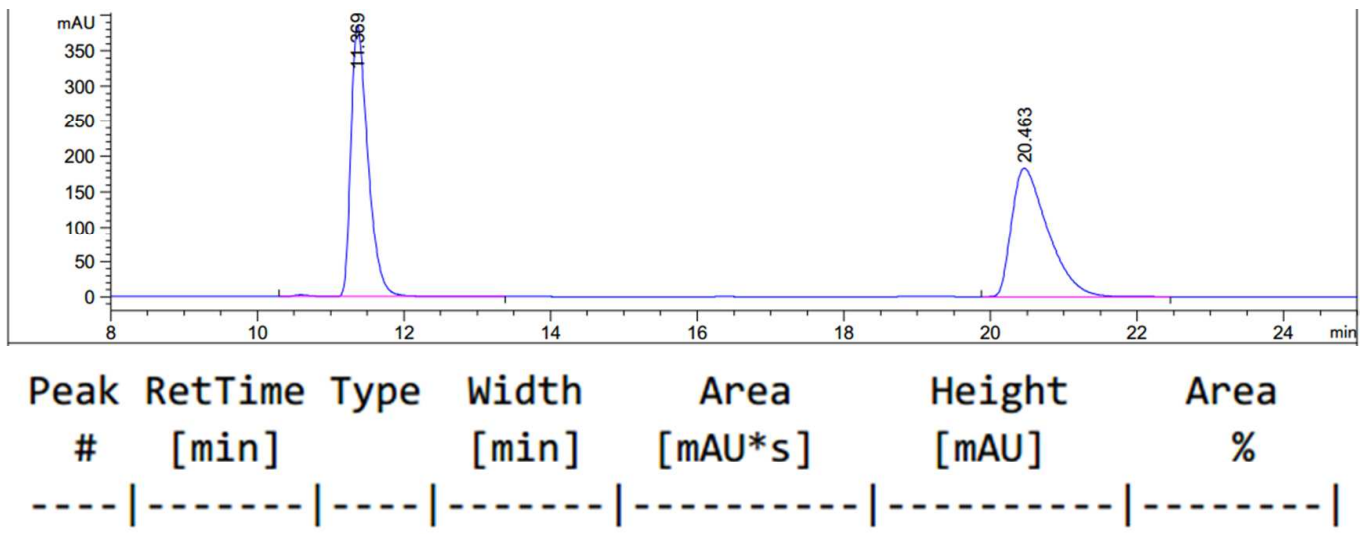

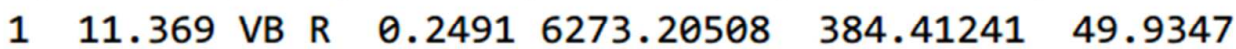

$\begin{array}{lllllll}2 & 20.463 & \text { BB } & 0.5251 & 6289.60156 & 182.92288 & 50.0653\end{array}$ 
HPLC trace for enantienriched-3aa (ee $=97 \%)$
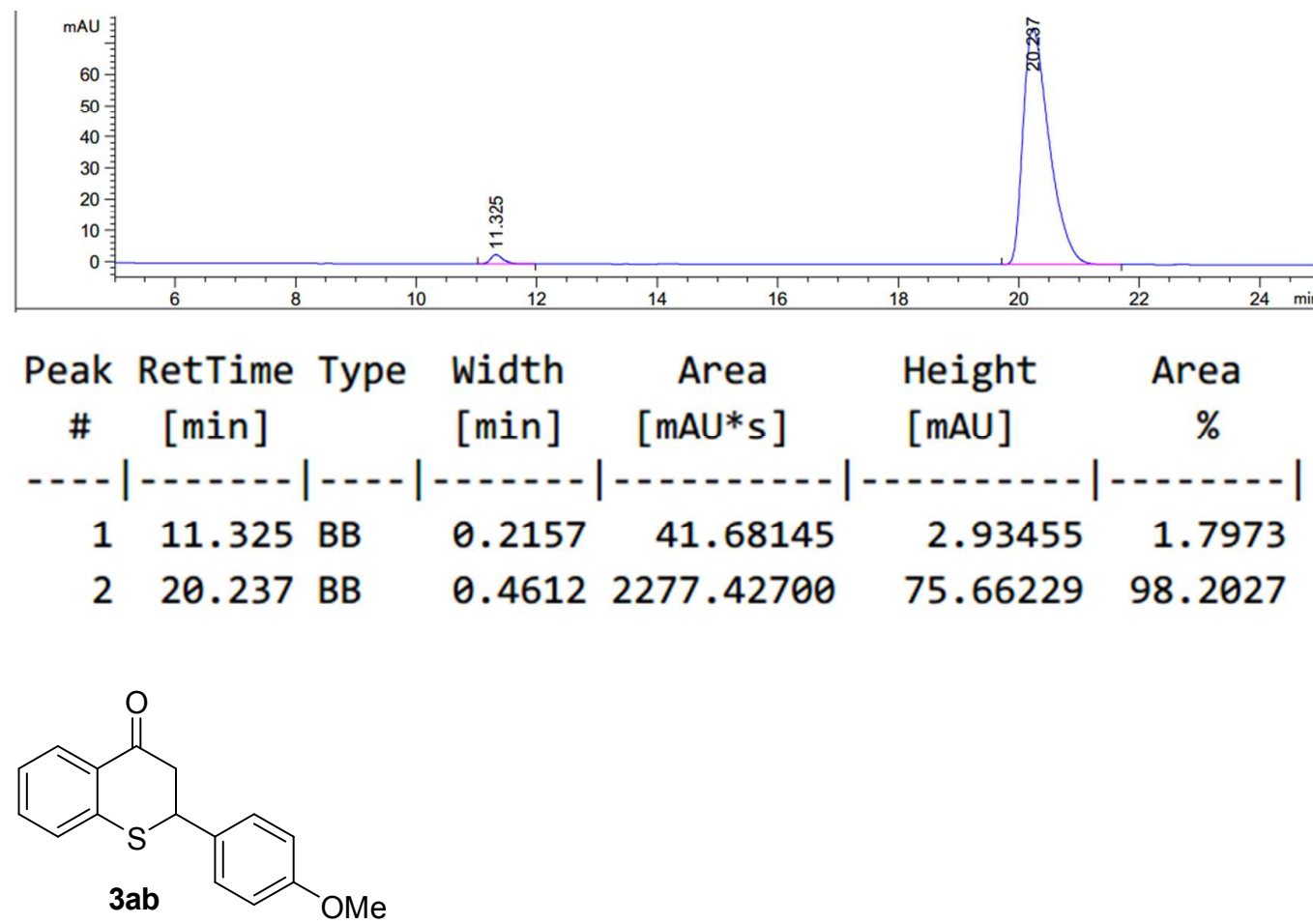

HPLC trace for racemic-3ab
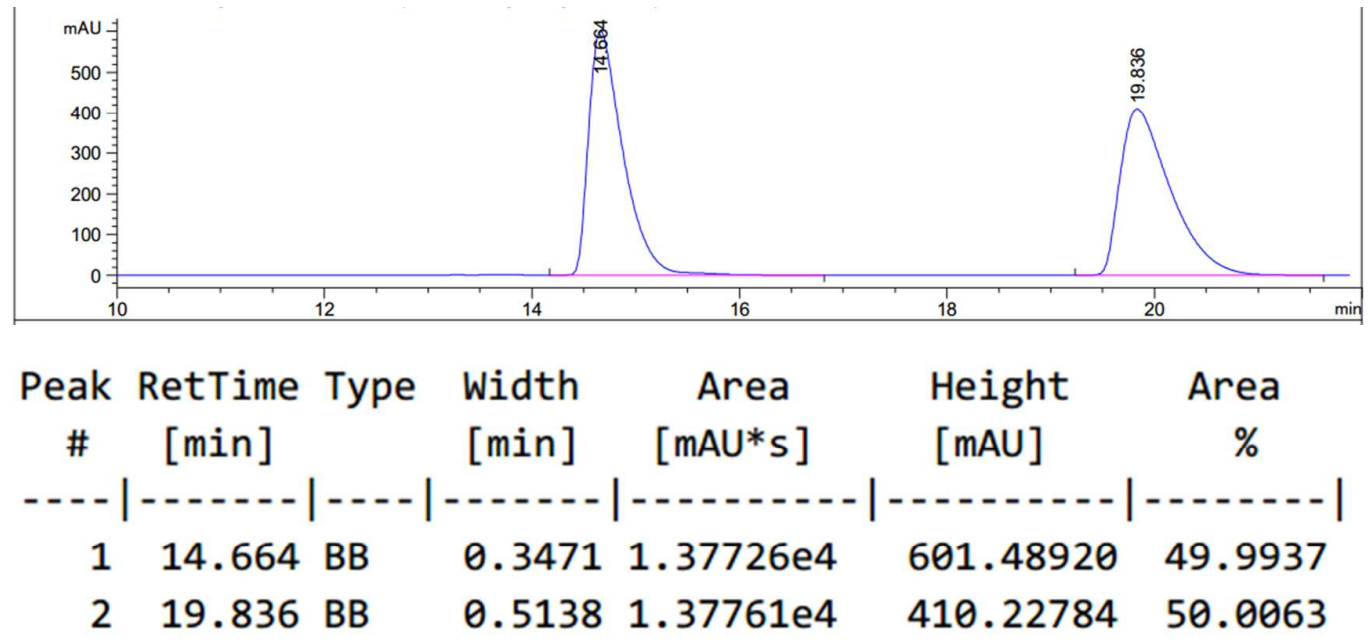

HPLC trace for enantienriched-3ab $(\mathrm{ee}=85 \%)$

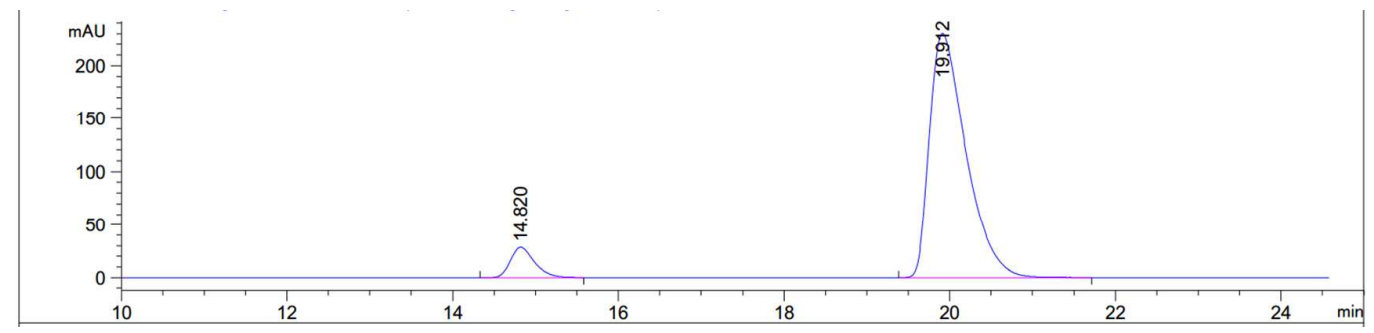



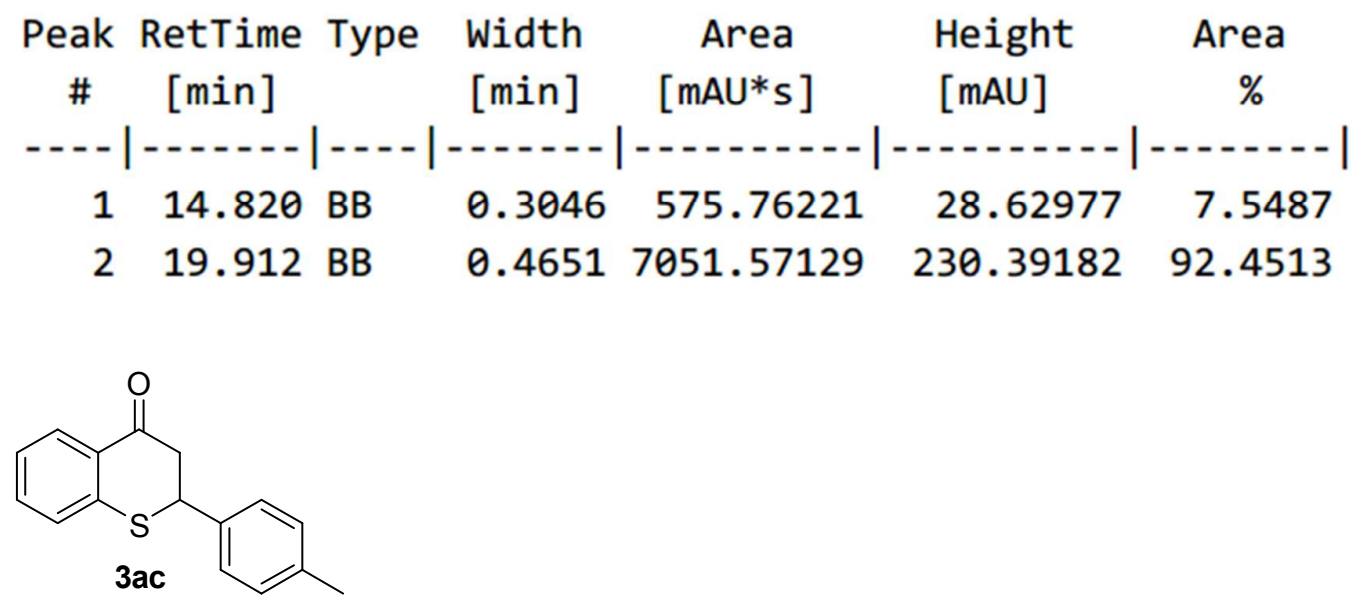

HPLC trace for racemic-3ac

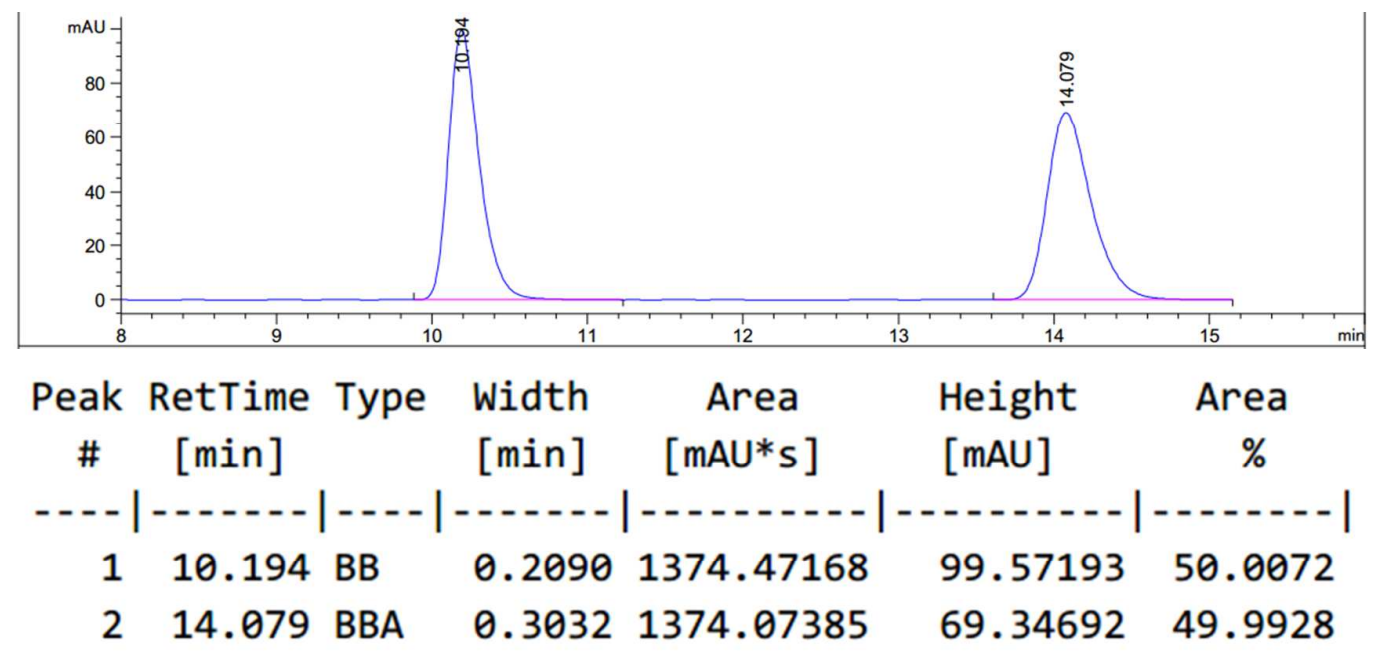

HPLC trace for enantienriched-3ac (ee $=94 \%)$

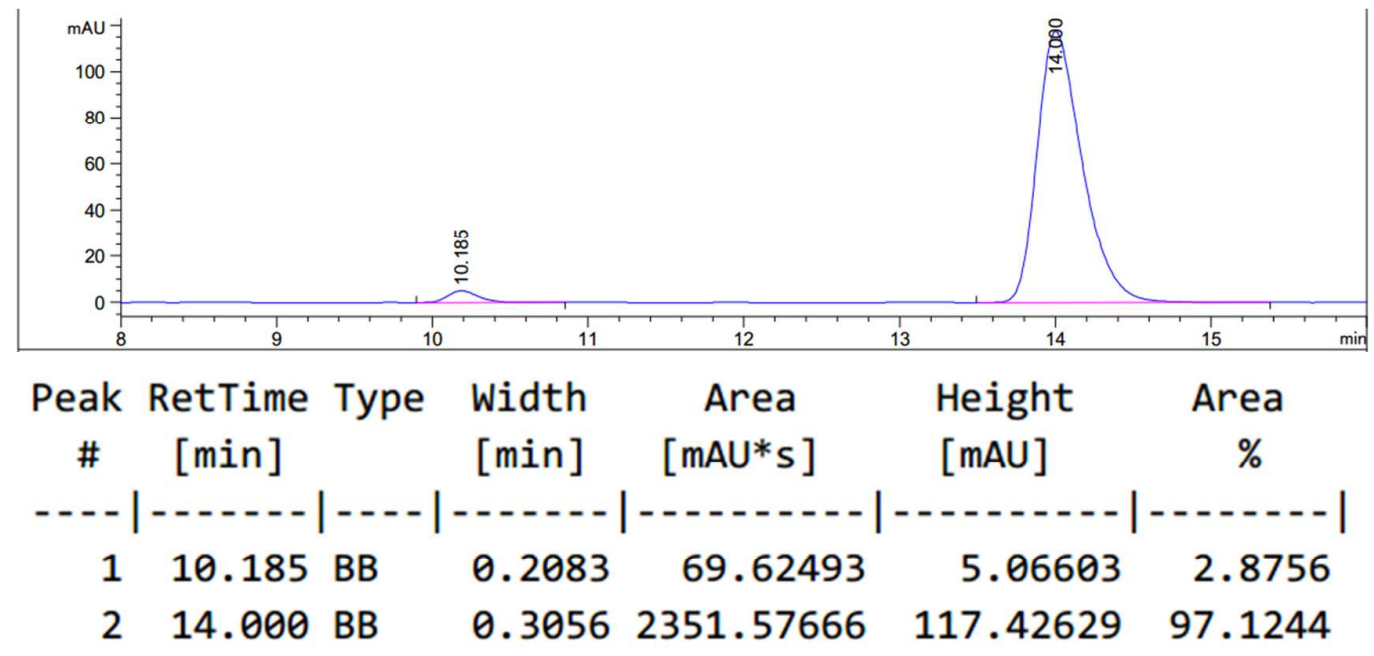




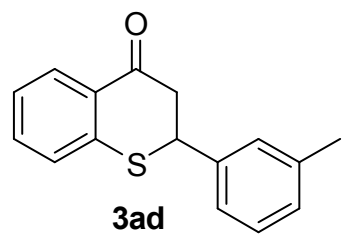

HPLC trace for racemic-3ad

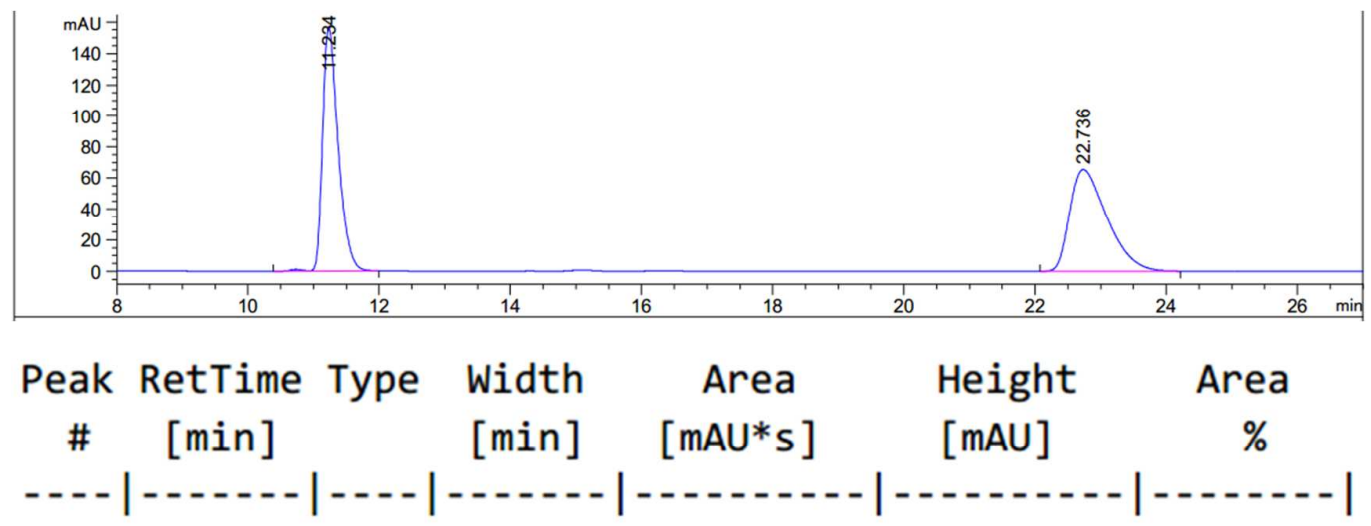

$\begin{array}{lllllll}1 & 11.234 & \text { VB R } & 0.2485 & 2575.02783 & 156.79295 & 49.9839\end{array}$

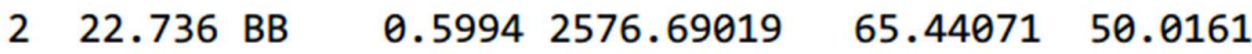

HPLC trace for enantienriched-3ad $(\mathrm{ee}=95 \%)$

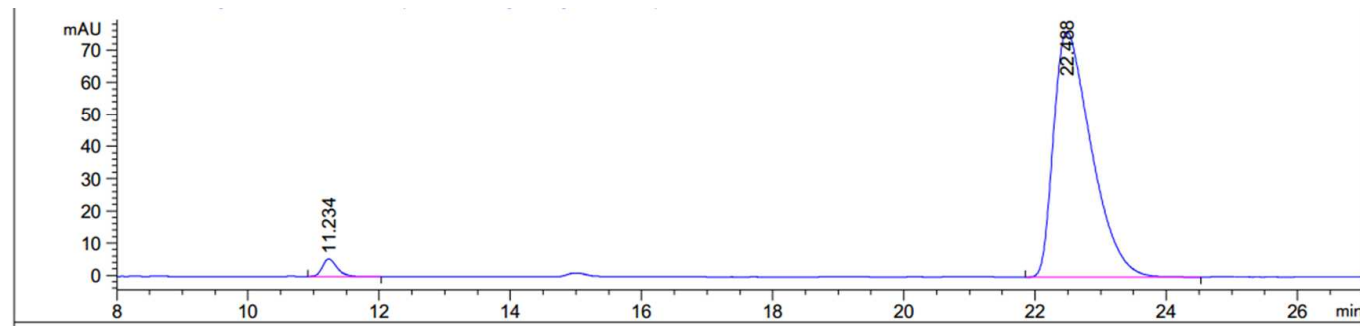

Peak RetTime Type Width Area Height Area

$\begin{array}{lllll}\# & {[\mathrm{~min}]} & {[\mathrm{min}]} & {[\mathrm{mAU} * \mathrm{~s}]} & {[\mathrm{mAU}]}\end{array}$

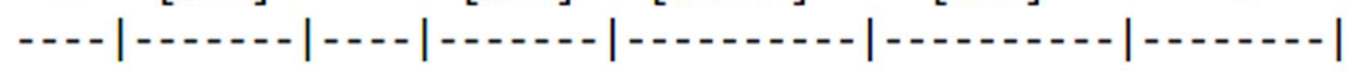
$111.234 \mathrm{BB}$
$\begin{array}{llll}0.2372 & 85.23732 & 5.48929 & 2.7262\end{array}$
222.488 BB
$0.6097 \quad 3041.41260 \quad 76.18979 \quad 97.2738$<smiles>Cc1ccccc1C1CC(=O)c2ccccc2S1</smiles>

HPLC trace for racemic-3ae 

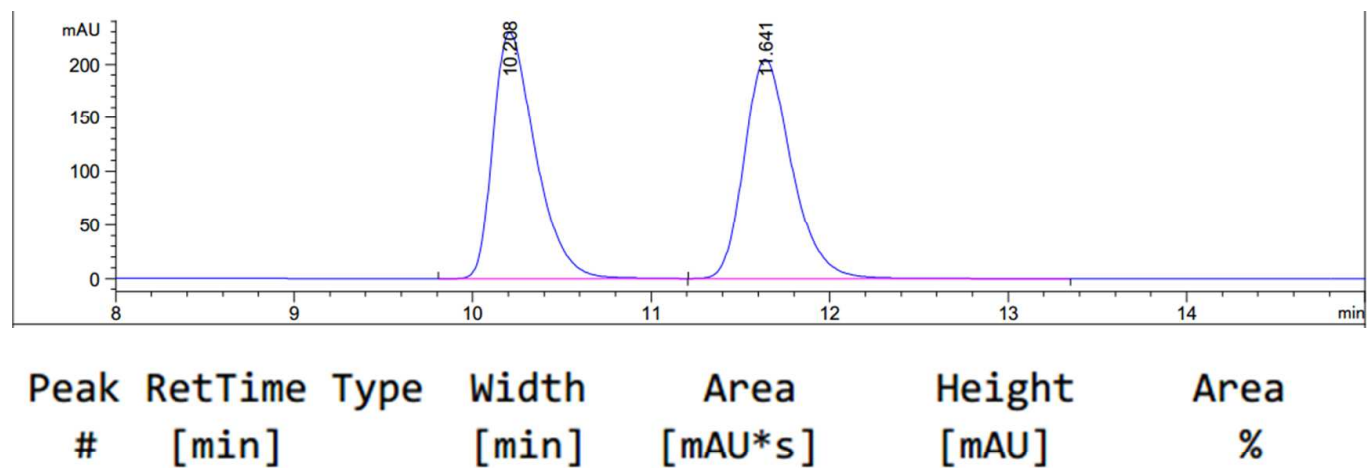

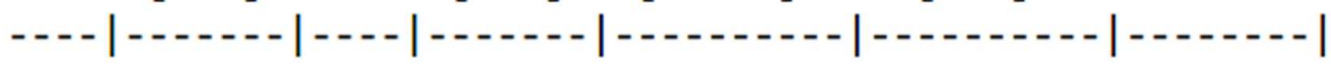

$\begin{array}{lllllll}1 & 10.208 & \text { BB } & 0.2524 & 3788.28784 & 229.66998 & 49.9912\end{array}$

$\begin{array}{lllllll}2 & 11.641 & \text { BB } & 0.2841 & 3789.62427 & 204.62364 & 50.0088\end{array}$

HPLC trace for enantienriched-3ae $(\mathrm{ee}=7 \%)$

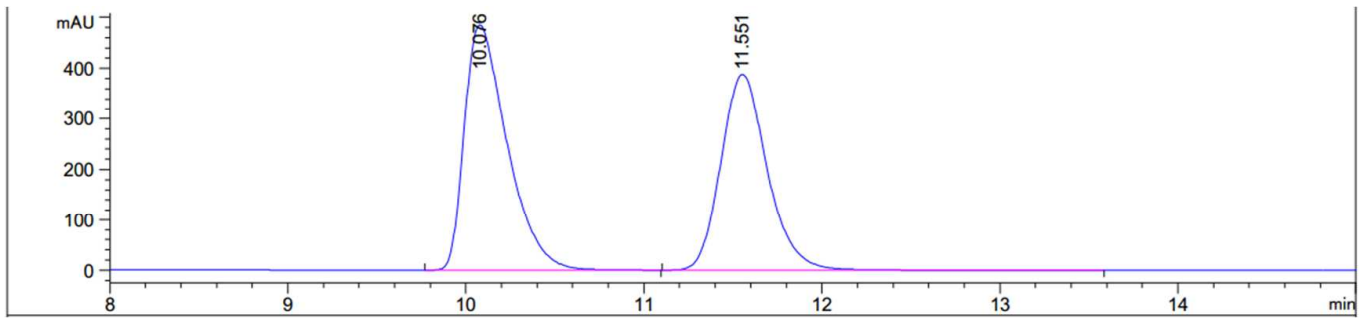

Peak RetTime Type width Area Height Area

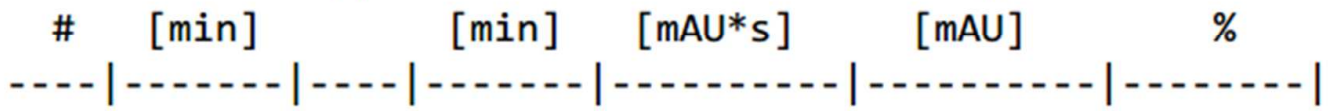

$1 \quad 10.076$ BB $\quad 0.2548 \quad 8091.01123 \quad 484.48355 \quad 53.5301$

$\begin{array}{lllllll}2 & 11.551 & \text { BB } & 0.2771 & 7023.86328 & 388.18030 & 46.4699\end{array}$<smiles>O=C1CC(c2ccc(F)cc2)Sc2ccccc21</smiles>

HPLC trace for racemic-3af

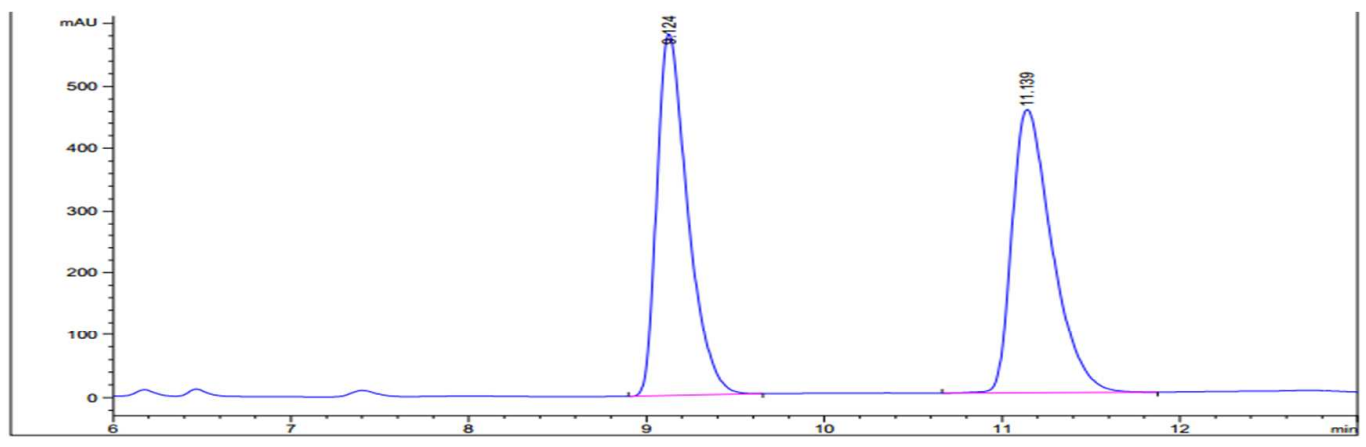




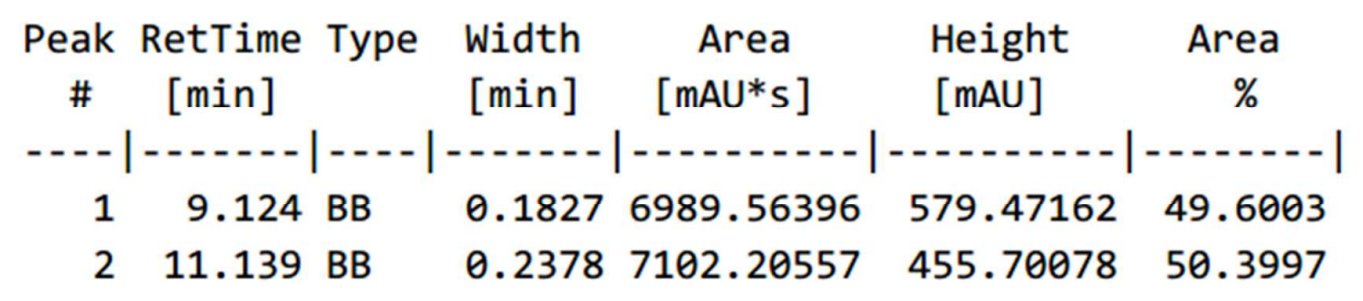

HPLC trace for enantienriched-3af (ee $=91 \%)$
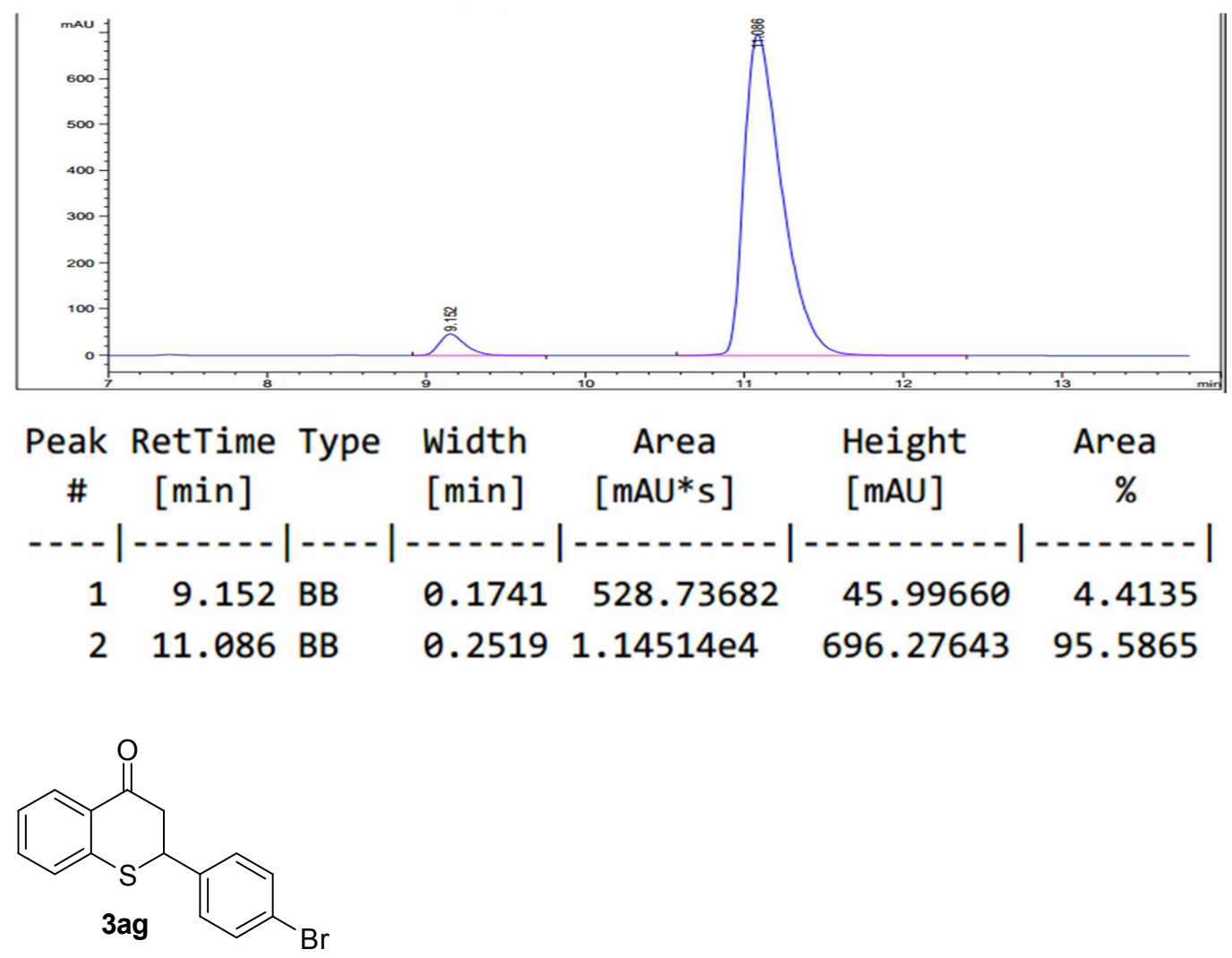

HPLC trace for racemic-3ag

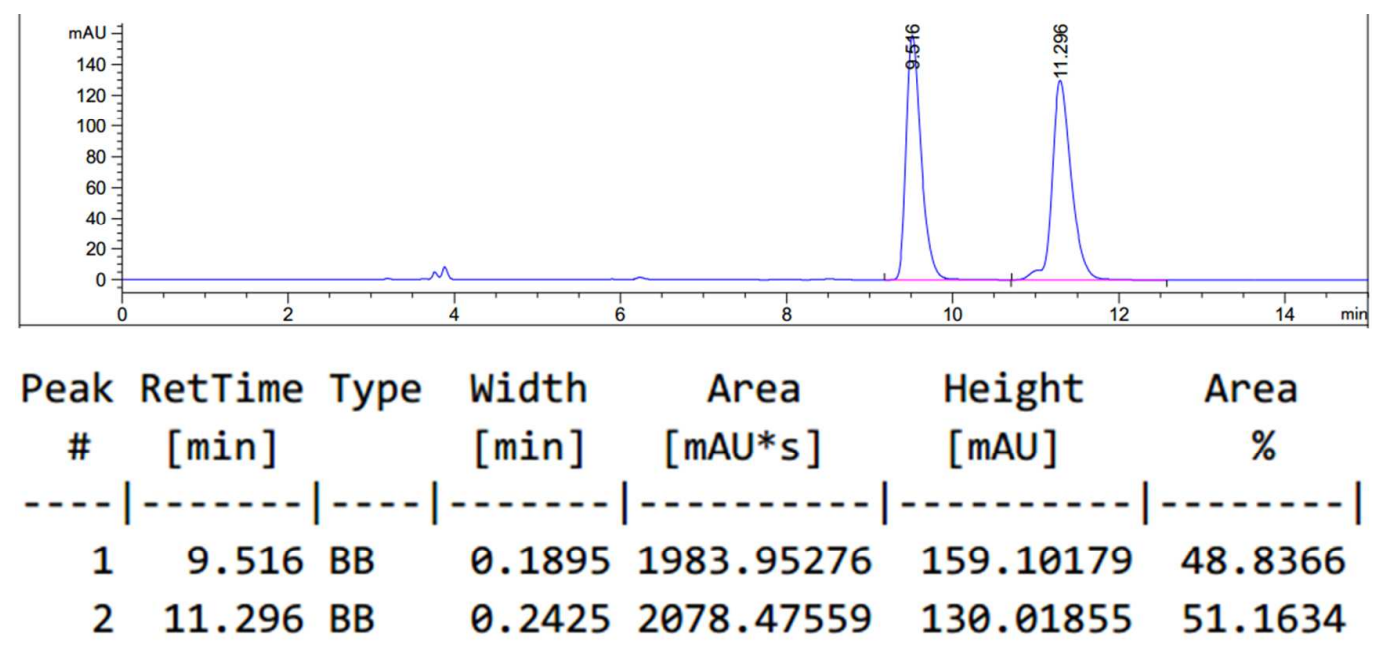


HPLC trace for enantienriched-3ag (ee $=95 \%)$

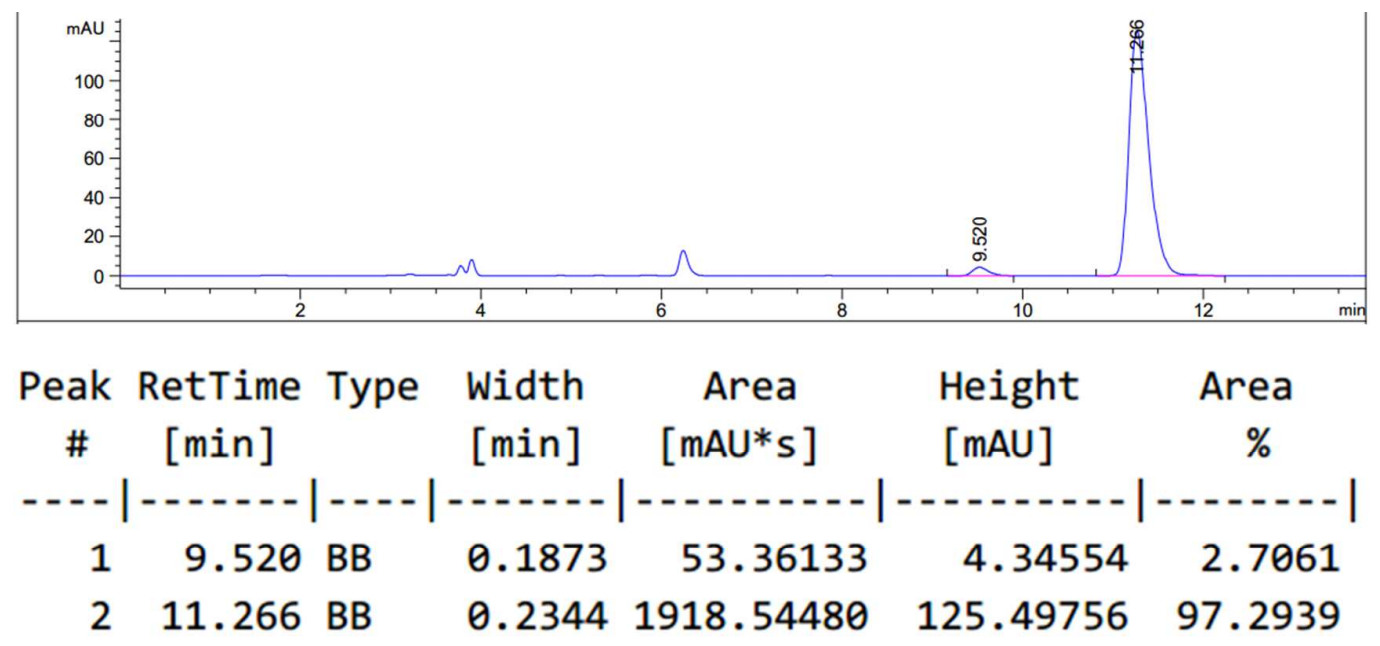<smiles>O=C1CC(c2ccc(C(F)(F)F)cc2)Sc2ccccc21</smiles>

HPLC trace for racemic-3ah

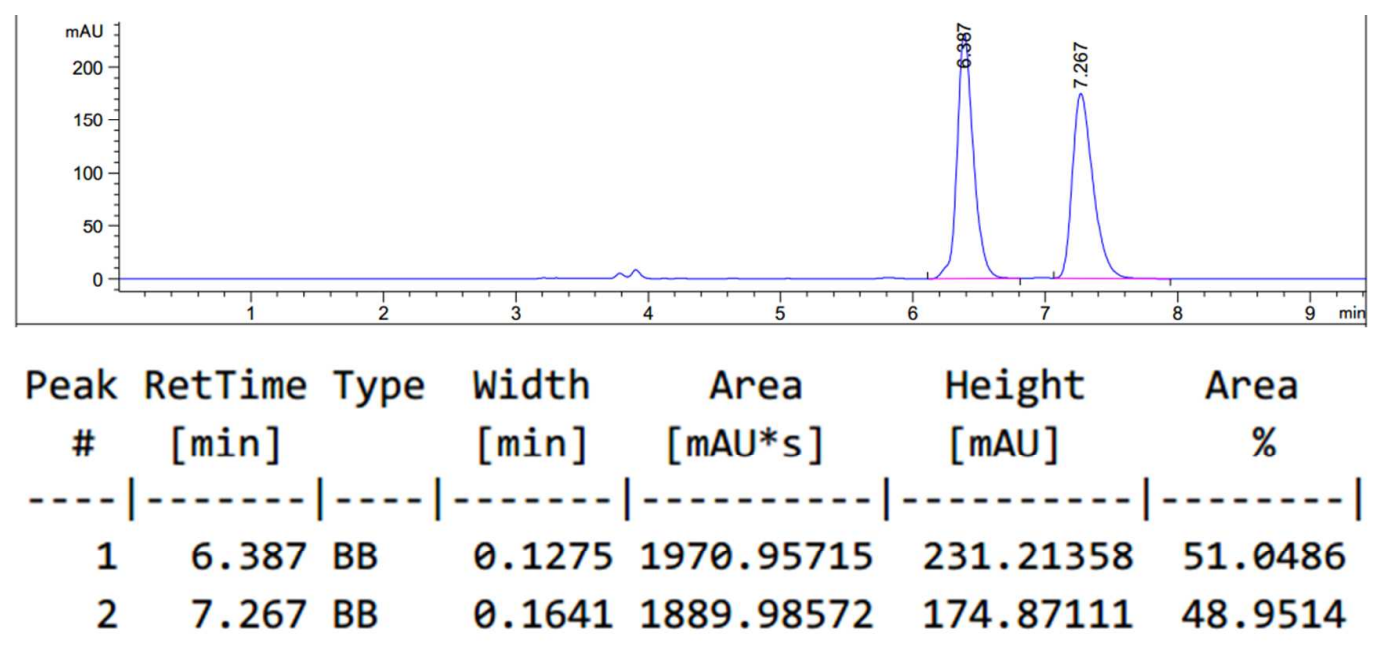

HPLC trace for enantienriched-3ah $(\mathrm{ee}=10 \%)$

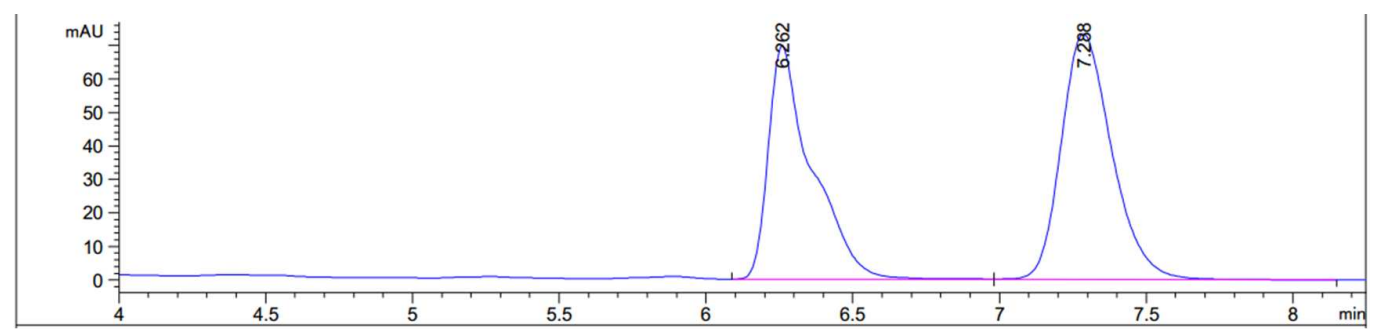




\begin{tabular}{|c|c|c|c|c|c|}
\hline $\begin{array}{c}\text { Peak } \\
\#\end{array}$ & $\begin{array}{l}\text { RetTime Type } \\
\text { [min] }\end{array}$ & $\begin{array}{l}\text { Width } \\
\text { [min] }\end{array}$ & $\begin{array}{c}\text { Area } \\
{\left[\mathrm{mAU}^{*} \mathrm{~s}\right]}\end{array}$ & $\begin{array}{l}\text { Height } \\
{[\mathrm{mAU}]}\end{array}$ & $\begin{array}{c}\text { Area } \\
\%\end{array}$ \\
\hline & & & & & \\
\hline 1 & 6.262 ВВ & 0.1439 & 717.05115 & 69.78755 & 44.9926 \\
\hline 2 & 7.288 BBA & 0.1837 & 876.65961 & 73.21841 & 55.0074 \\
\hline
\end{tabular}<smiles>Cc1cc(C)cc(C2CC(=O)c3ccccc3S2)c1</smiles>

HPLC trace for racemic-3ai

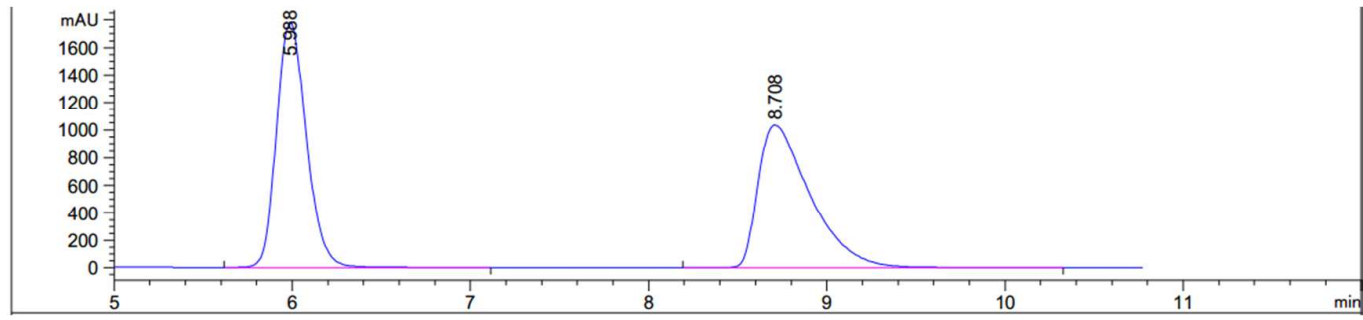

\begin{tabular}{cccccc}
$\begin{array}{c}\text { Peak RetTime Type } \\
\text { \# } \\
{[\text { win] }}\end{array}$ & $\begin{array}{c}\text { Width } \\
{[\text { min }]}\end{array}$ & $\begin{array}{c}\text { Area } \\
{[\text { mAU*s }]}\end{array}$ & $\begin{array}{c}\text { Height } \\
{[\mathrm{mAU}]}\end{array}$ & $\begin{array}{c}\text { Area } \\
\%\end{array}$ \\
\hline 1 & 5.988 BB & 0.1819 & $2.07843 e 4$ & 1783.70630 & 49.6069 \\
2 & 8.708 BB & 0.3064 & $2.11137 e 4$ & 1033.36121 & 50.3931
\end{tabular}

HPLC trace for enantienriched-3ai (ee $=95 \%)$

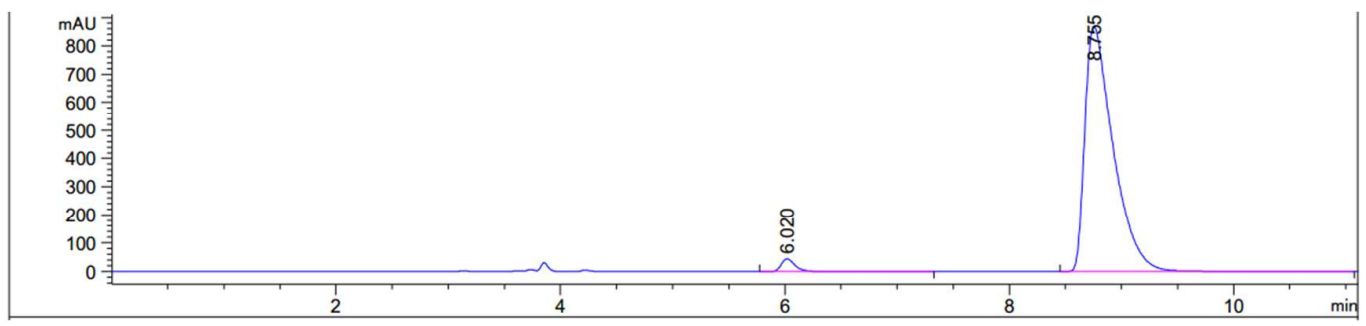

\begin{tabular}{|c|c|c|c|c|c|c|}
\hline $\begin{array}{c}\text { Peak } \\
\text { \# }\end{array}$ & $\begin{array}{c}\text { RetTime } \\
\text { [min] }\end{array}$ & Type & $\begin{array}{l}\text { Width } \\
\text { [min] }\end{array}$ & $\begin{array}{c}\text { Area } \\
{\left[\mathrm{mAU}^{*} \mathrm{~s}\right]}\end{array}$ & $\begin{array}{l}\text { Height } \\
{[\mathrm{mAU}]}\end{array}$ & $\begin{array}{c}\text { Area } \\
\%\end{array}$ \\
\hline & & & & --1 & -- & -4 \\
\hline 1 & 6.020 & BB & 0.1285 & 374.15665 & 44.32750 & 2.3962 \\
\hline 2 & 8.755 & BBA & 0.2606 & $1.52404 \mathrm{e} 4$ & 869.14746 & 97.6038 \\
\hline
\end{tabular}




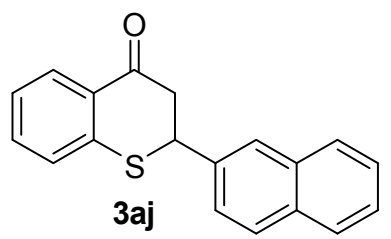

HPLC trace for racemic-3aj
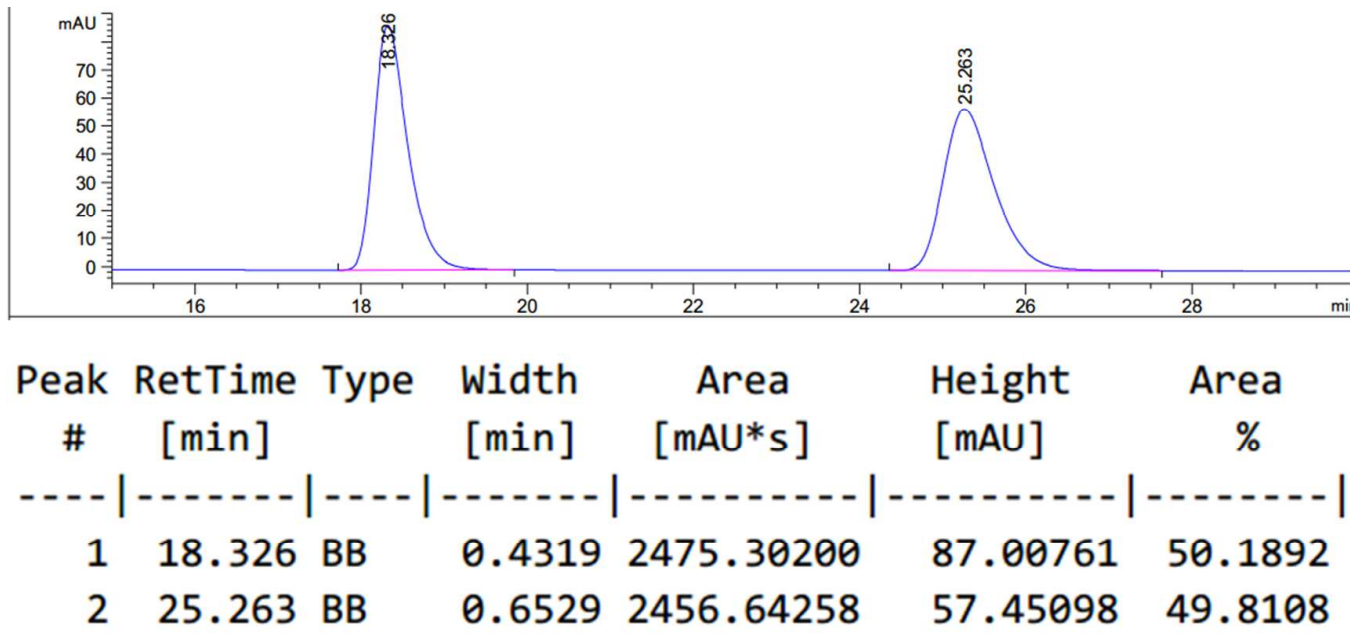

HPLC trace for enantienriched-3aj (ee $=95 \%)$

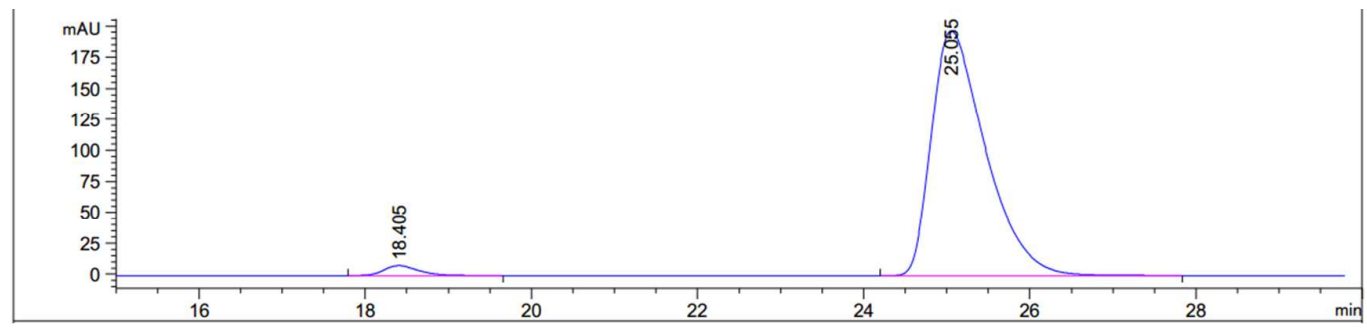

\begin{tabular}{cccccc}
$\begin{array}{c}\text { Peak RetTime Type } \\
\text { Width }\end{array}$ & $\begin{array}{c}\text { Area } \\
\text { [min] }\end{array}$ & $\begin{array}{c}\text { Height } \\
{[\mathrm{mAU} * \mathrm{~m}]}\end{array}$ & $\begin{array}{c}\text { Area } \\
{[\mathrm{mAU}]}\end{array}$ & $\%$ \\
\hline 1 & 18.405 BB & 0.4373 & 229.05066 & 7.96969 & 2.5039 \\
2 & 25.055 BB & 0.6876 & 8918.75195 & 197.21492 & 97.4961
\end{tabular}<smiles>O=C1CC(c2ccco2)Sc2ccccc21</smiles>

HPLC trace for racemic-3ak 

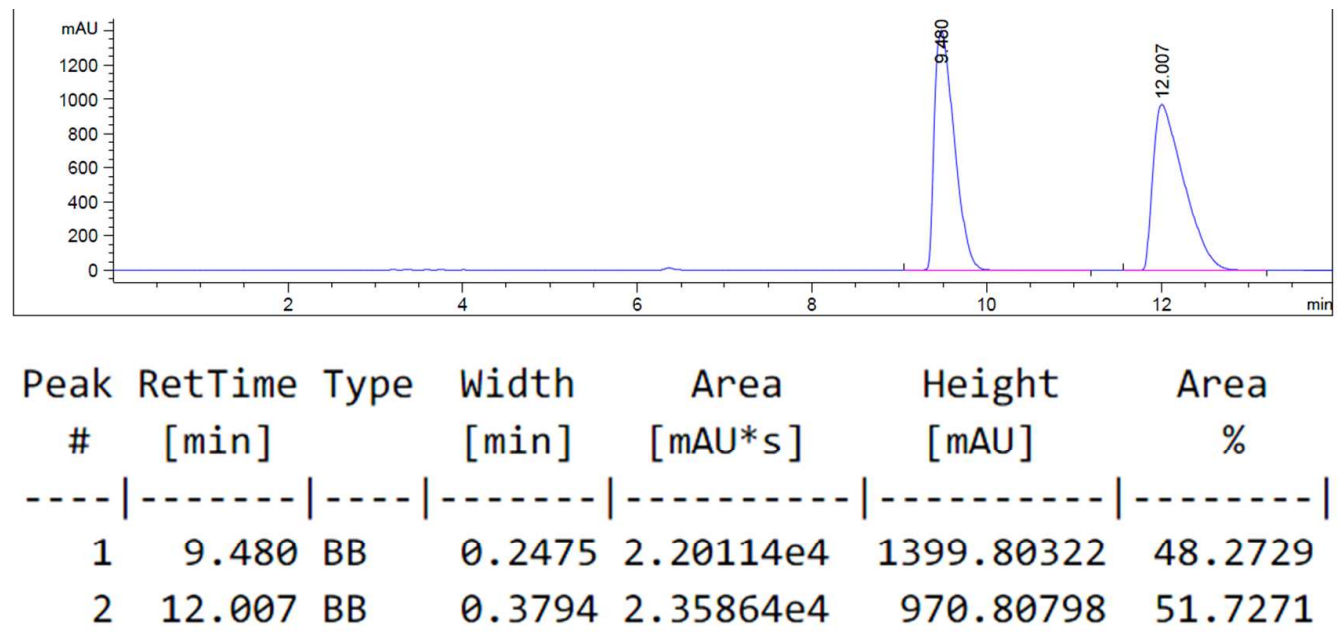

HPLC trace for enantienriched-3ak $(\mathrm{ee}=4 \%)$

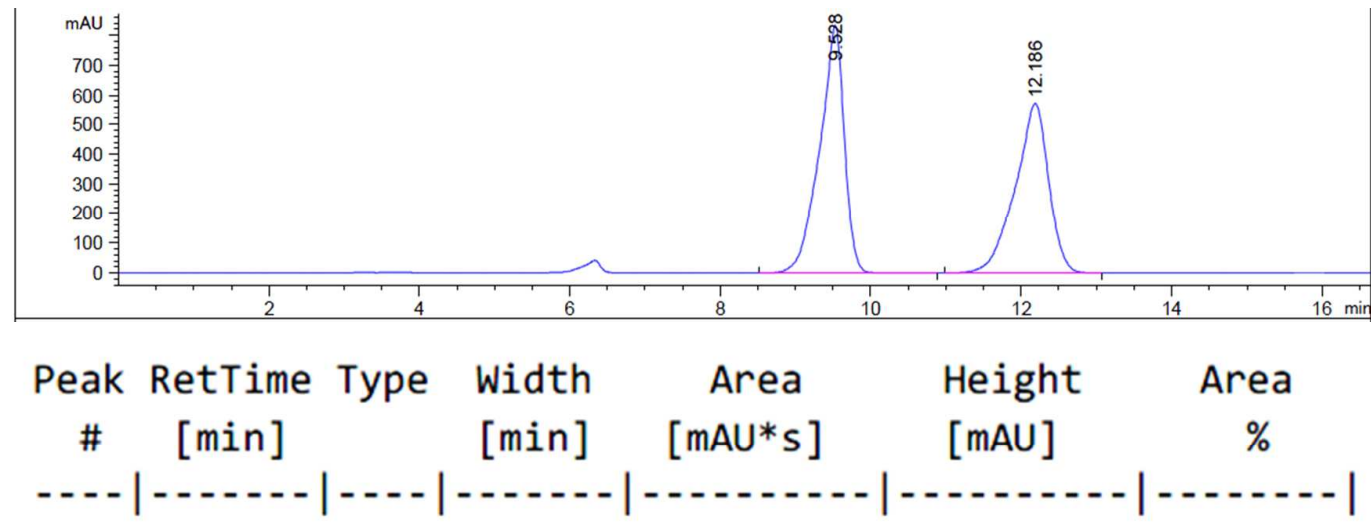

$\begin{array}{llllll}1 & 9.528 \text { BB } & 0.3209 & 1.90206 \mathrm{e} & 831.53711 & 51.9102\end{array}$

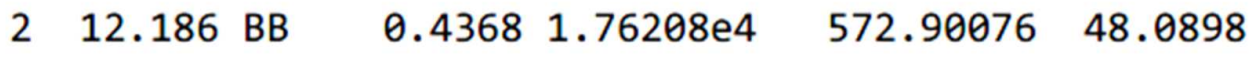<smiles>O=C1CC(c2cccs2)Sc2ccccc21</smiles>

HPLC trace for racemic-3al

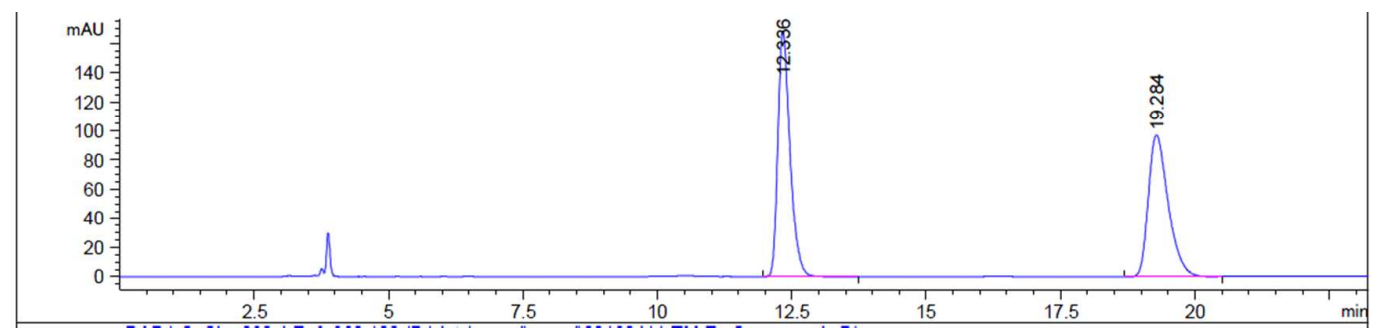




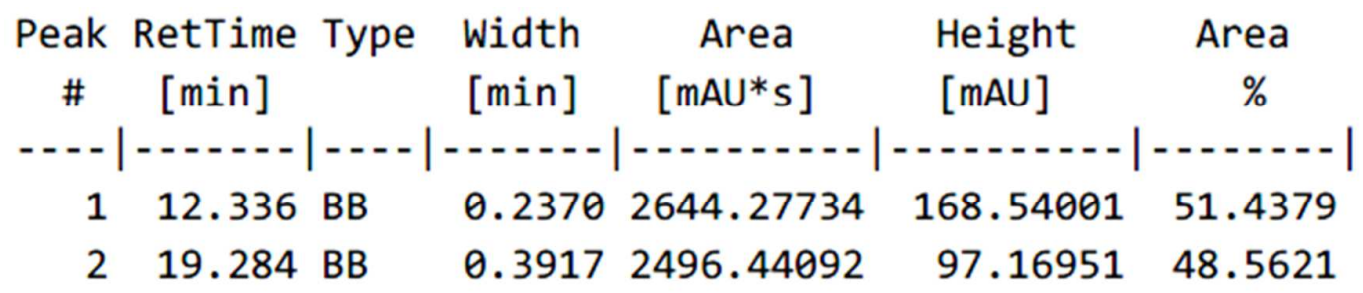

HPLC trace for enantienriched-3al (ee $=2 \%)$

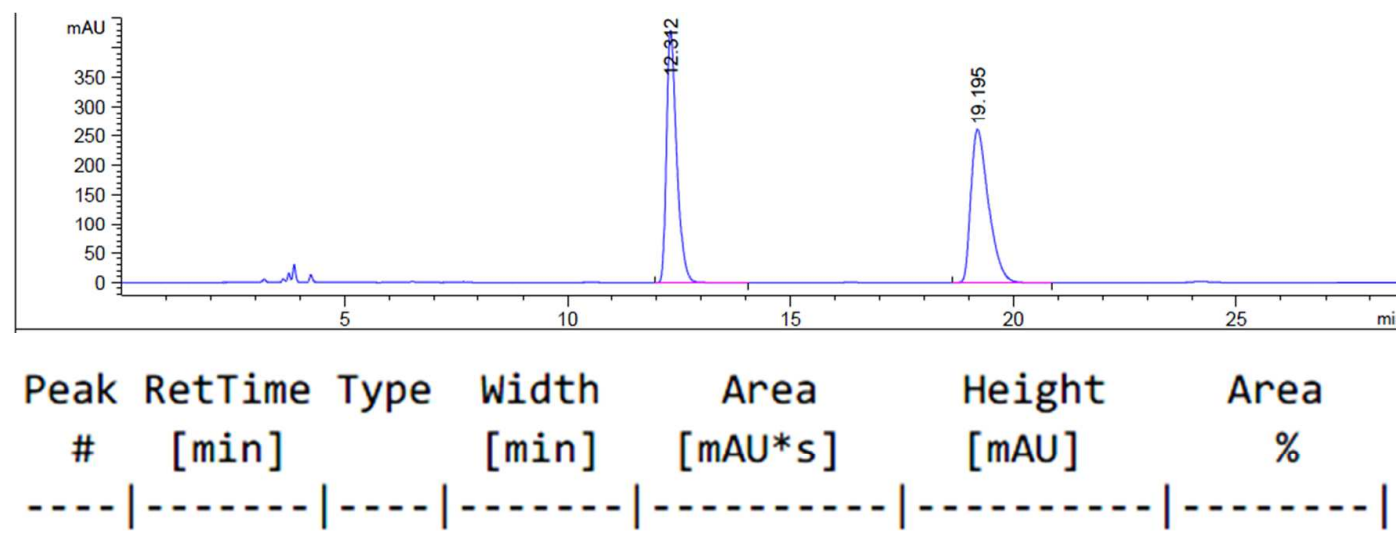
$112.312 \mathrm{BB}$
$0.24216862 .95801 \quad 430.17783 \quad 48.9613$
$219.195 \mathrm{BB}$
$\begin{array}{llll}0.4204 & 7154.16260 & 260.53558 \quad 51.0387\end{array}$<smiles>COc1ccc2c(c1)C(=O)CC(c1ccccc1)S2</smiles>

HPLC trace for racemic-3ba
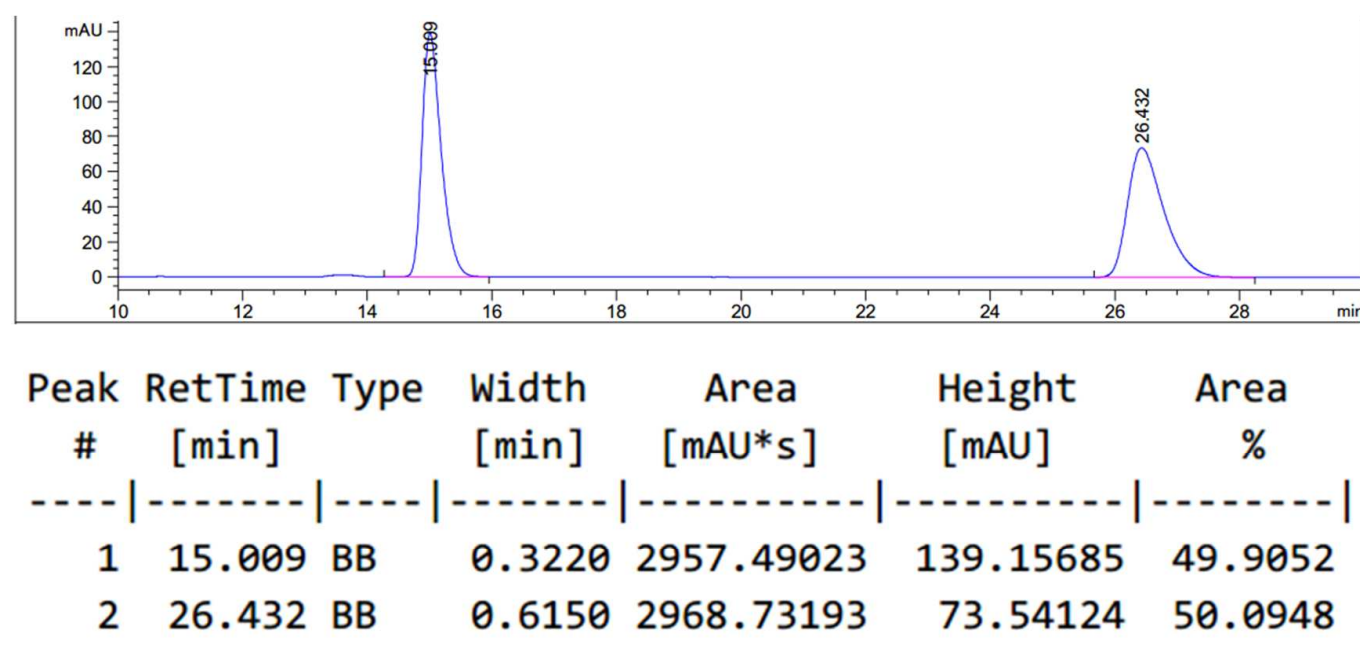

HPLC trace for enantienriched-3ba $(\mathrm{ee}=94 \%)$ 

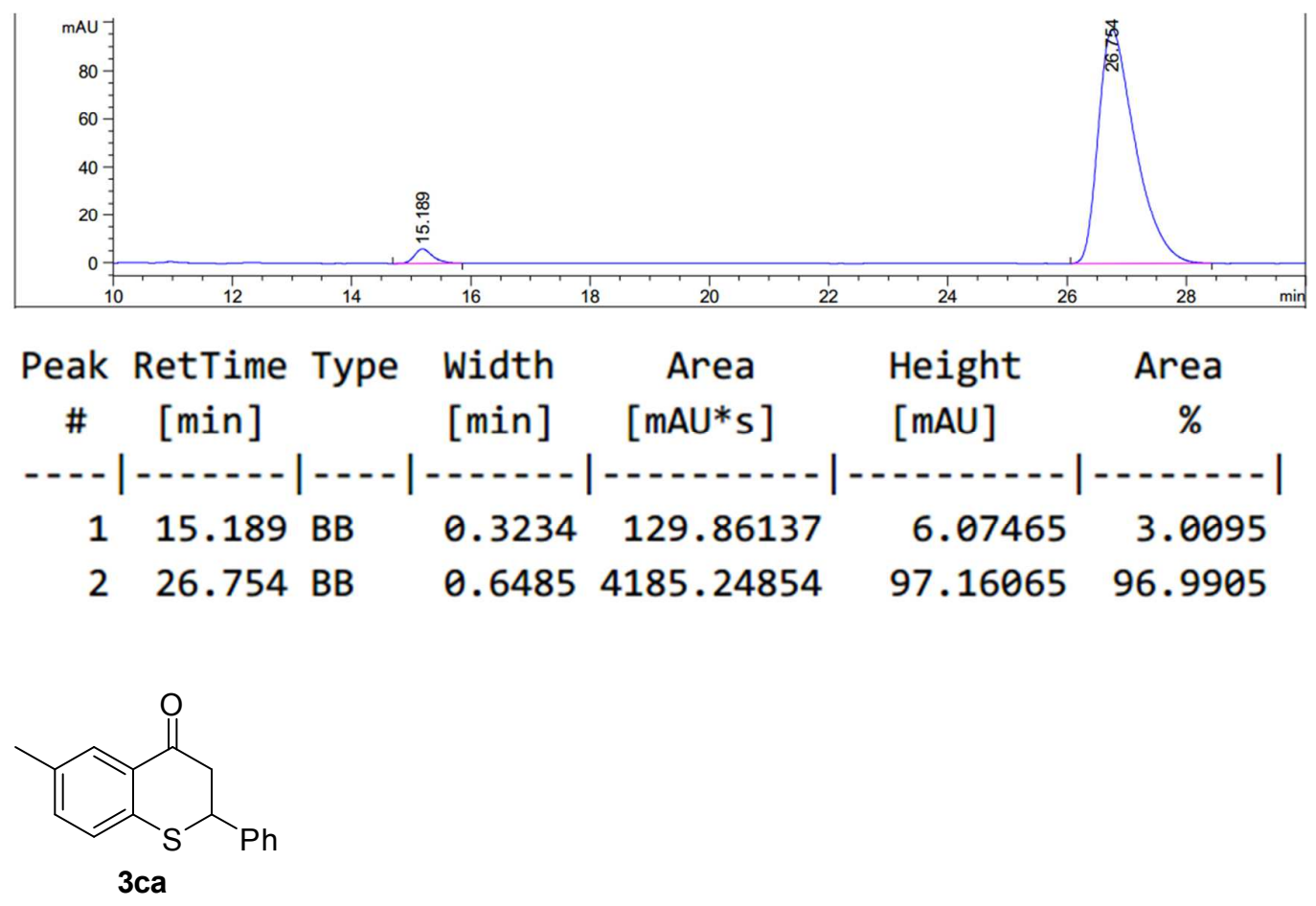

HPLC trace for racemic-3ca
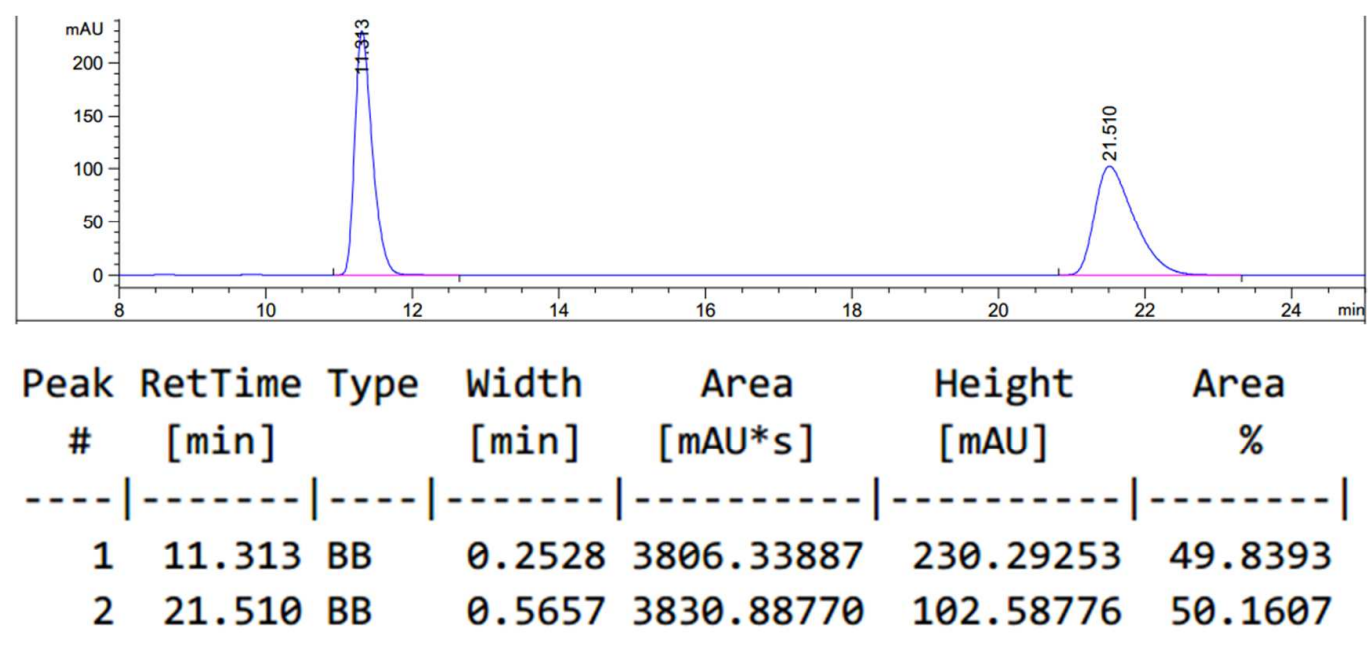

HPLC trace for enantienriched-3ca (ee $=95 \%)$

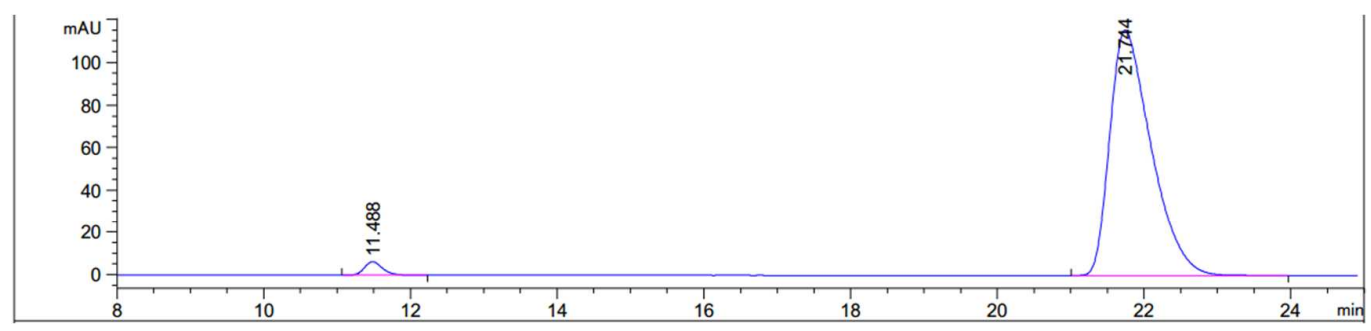




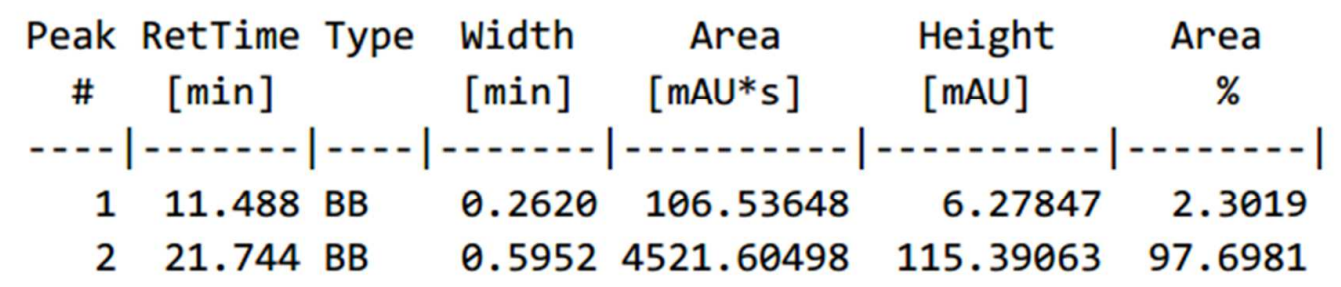<smiles>O=C1CC(c2ccccc2)Sc2ccc(F)cc21</smiles>

HPLC trace for racemic-3da

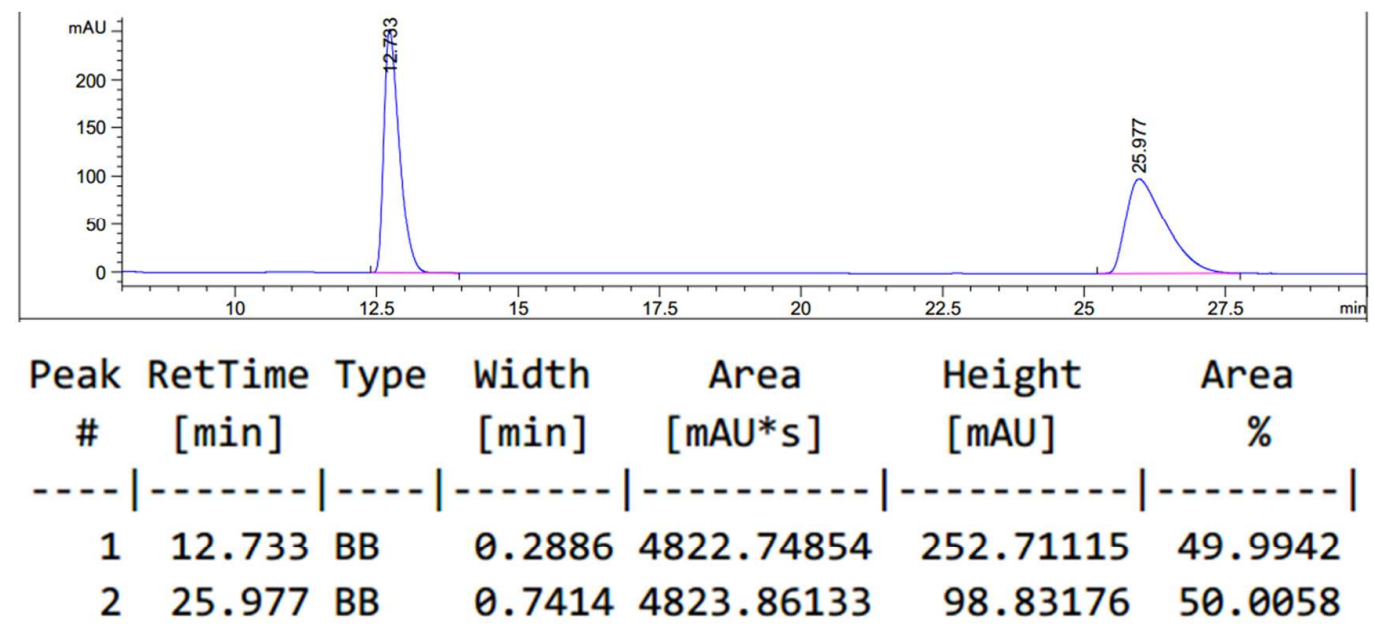

HPLC trace for enantienriched-3da $(\mathrm{ee}=93 \%)$

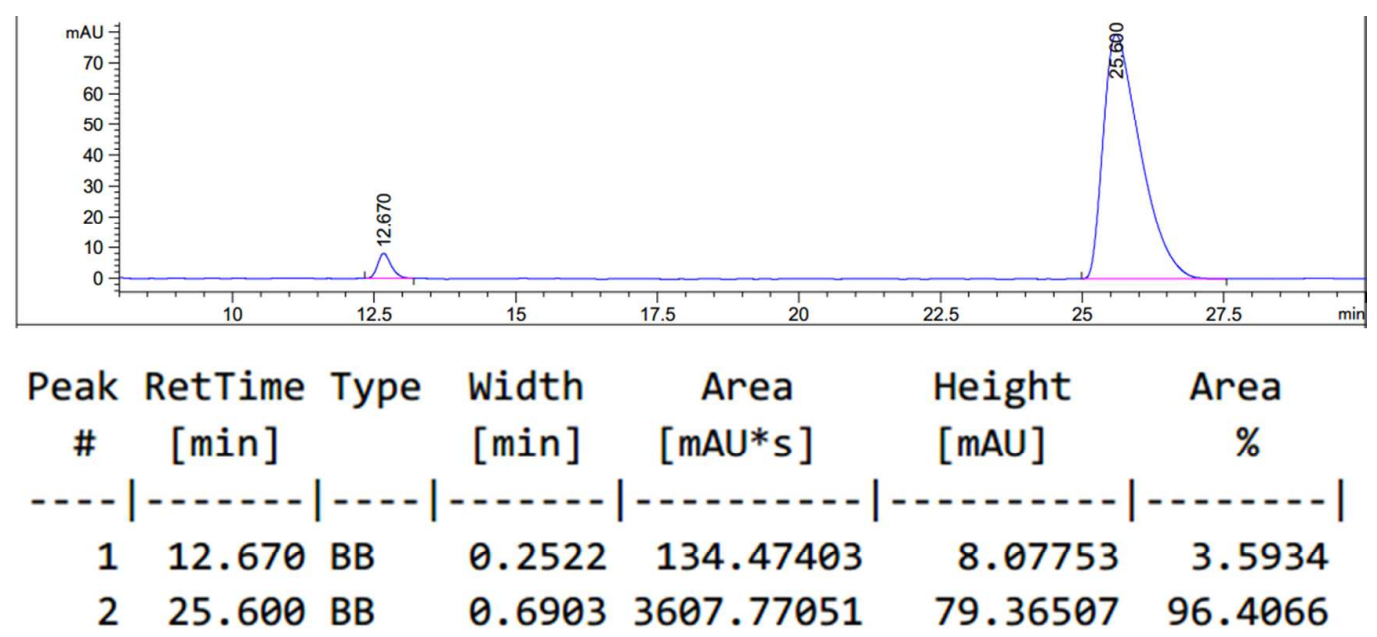


<smiles>O=C1CC(c2ccccc2)Sc2ccc(Cl)cc21</smiles>

HPLC trace for racemic-3ea

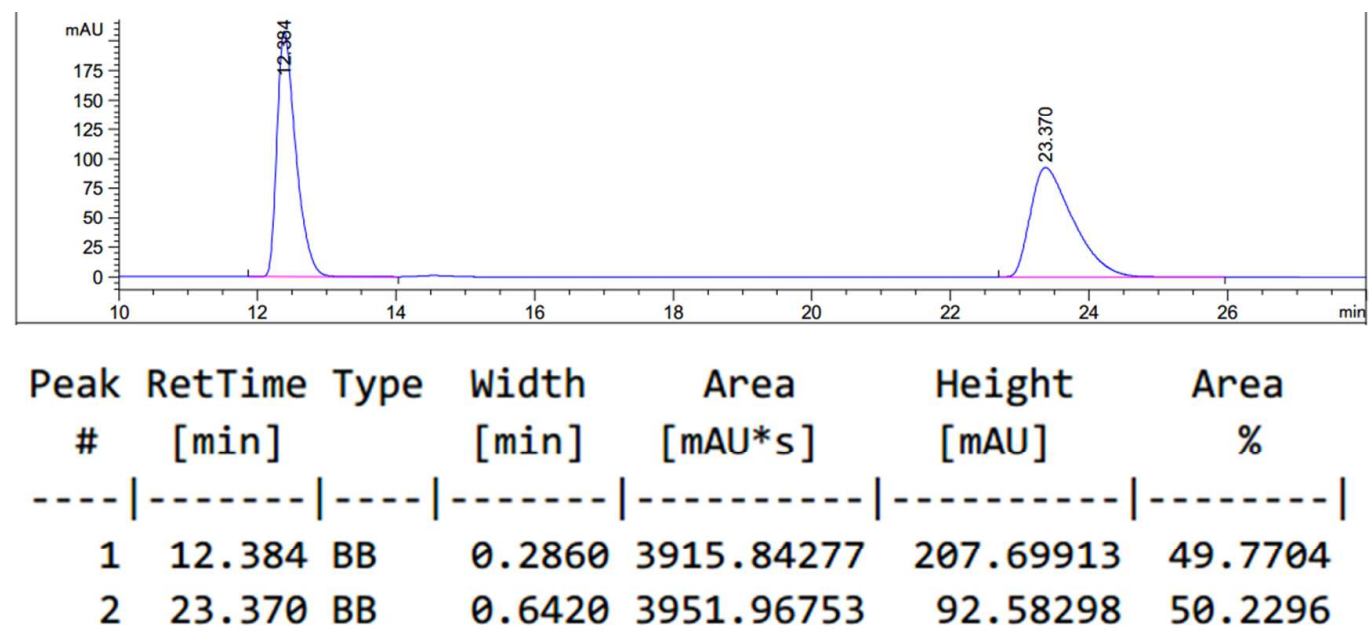

HPLC trace for enantienriched-3ea $(\mathrm{ee}=97 \%)$

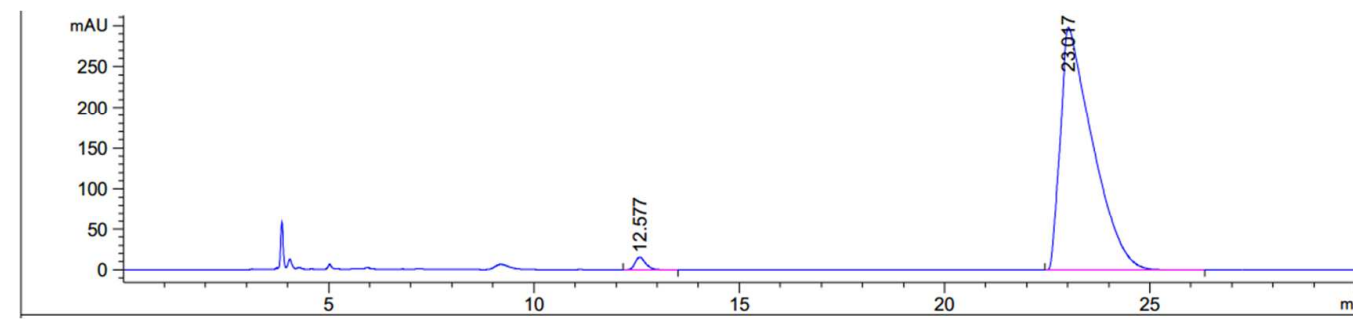

\begin{tabular}{|c|c|c|c|c|c|c|}
\hline $\begin{array}{c}\text { Peak } \\
\#\end{array}$ & $\begin{array}{c}\text { RetTime } \\
\text { [min] }\end{array}$ & Type & $\begin{array}{l}\text { Width } \\
\text { [min] }\end{array}$ & $\begin{array}{c}\text { Area } \\
{\left[\mathrm{mAU}^{*} \mathrm{~s}\right]}\end{array}$ & $\begin{array}{l}\text { Height } \\
{[\mathrm{mAU}]}\end{array}$ & $\begin{array}{c}\text { Area } \\
\%\end{array}$ \\
\hline & & & & 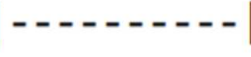 & & . \\
\hline 1 & 12.577 & BB & 0.2701 & 277.95413 & 15.58475 & 1.6790 \\
\hline 2 & 23.017 & BB & .7590 & $1.62770 \mathrm{e} 4$ & 298.11703 & 98.3210 \\
\hline
\end{tabular}<smiles>O=C1CC(c2ccccc2)Sc2ccc(Br)cc21</smiles>

HPLC trace for racemic-3fa 


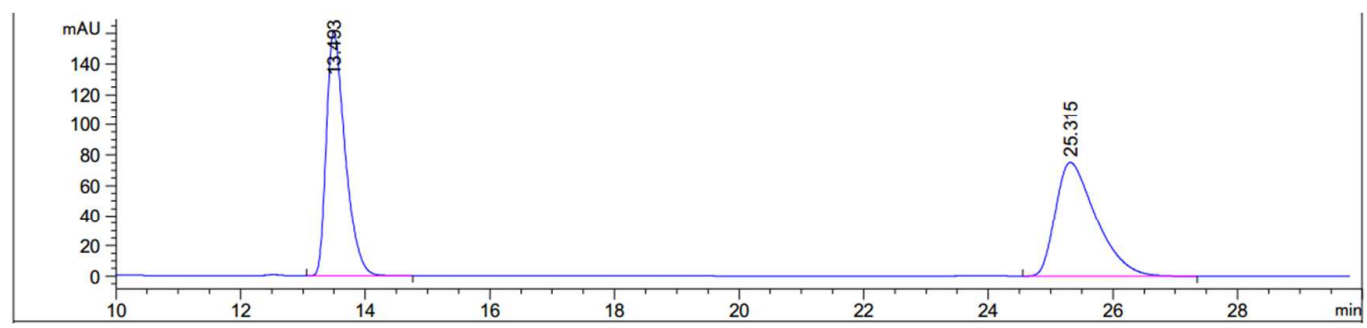
\# [min] [min] [mAU*s] [mAU]

$\begin{array}{lllllll}1 & 13.493 & \text { BB } & 0.3160 & 3374.75879 & 161.33435 & 49.8157\end{array}$

$\begin{array}{lllllll}2 & 25.315 & \text { BB } & 0.6876 & 3399.73120 & 75.17027 & 50.1843\end{array}$

HPLC trace for enantienriched-3fa $(e e=96 \%)$

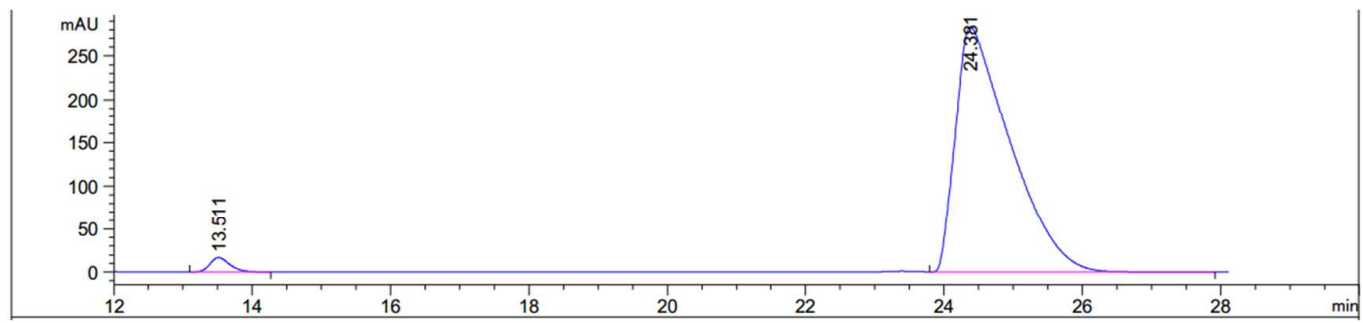

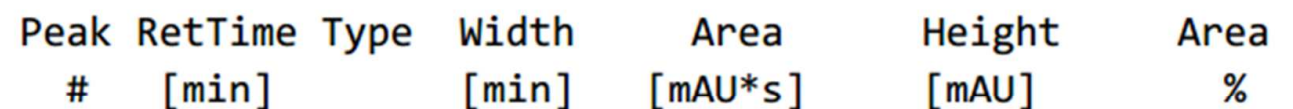

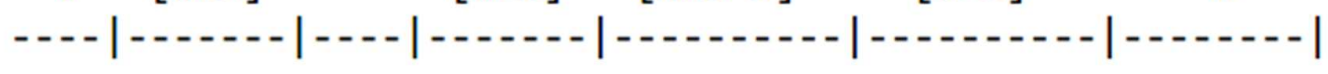
$113.511 \mathrm{BB}$
$0.3002 \quad 327.62231$
16.60175
2.0592
224.381 BB
$0.7907 \quad 1.55829 \mathrm{e} 4 \quad 282.85135 \quad 97.9408$<smiles>O=C1CC(c2ccccc2)Sc2ccc(C(F)(F)F)cc21</smiles>

HPLC trace for racemic-3ga

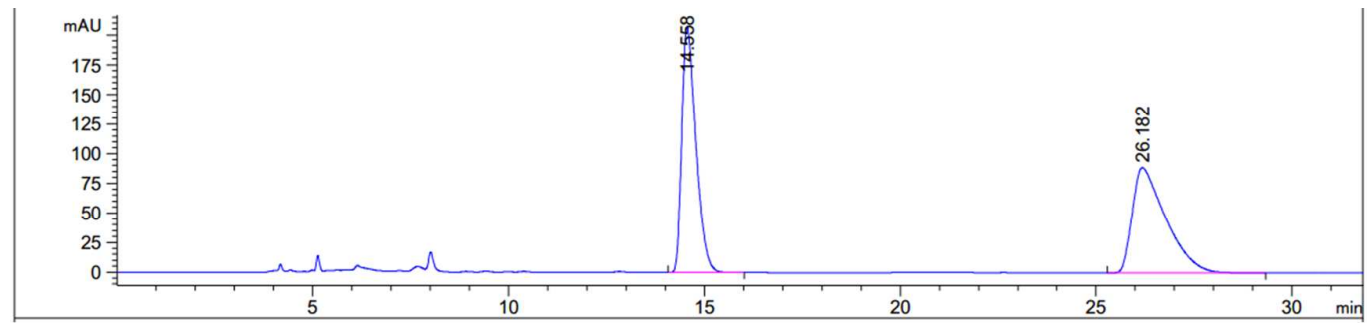




\begin{tabular}{cccccc}
$\begin{array}{c}\text { Peak RetTime Type } \\
\text { [min] }\end{array}$ & $\begin{array}{c}\text { Width } \\
{[\text { min] }}\end{array}$ & $\begin{array}{c}\text { Area } \\
{[\text { mAU*s] }}\end{array}$ & $\begin{array}{c}\text { Height } \\
{[\mathrm{mAU}]}\end{array}$ & $\begin{array}{c}\text { Area } \\
\%\end{array}$ \\
\hdashline 1 & 14.558 BB & 0.3781 & 5183.25098 & 207.04260 & 49.6616 \\
2 & 26.182 BB & 0.8561 & 5253.88770 & 88.81644 & 50.3384
\end{tabular}

HPLC trace for enantienriched-3ga $(\mathrm{ee}=91 \%)$
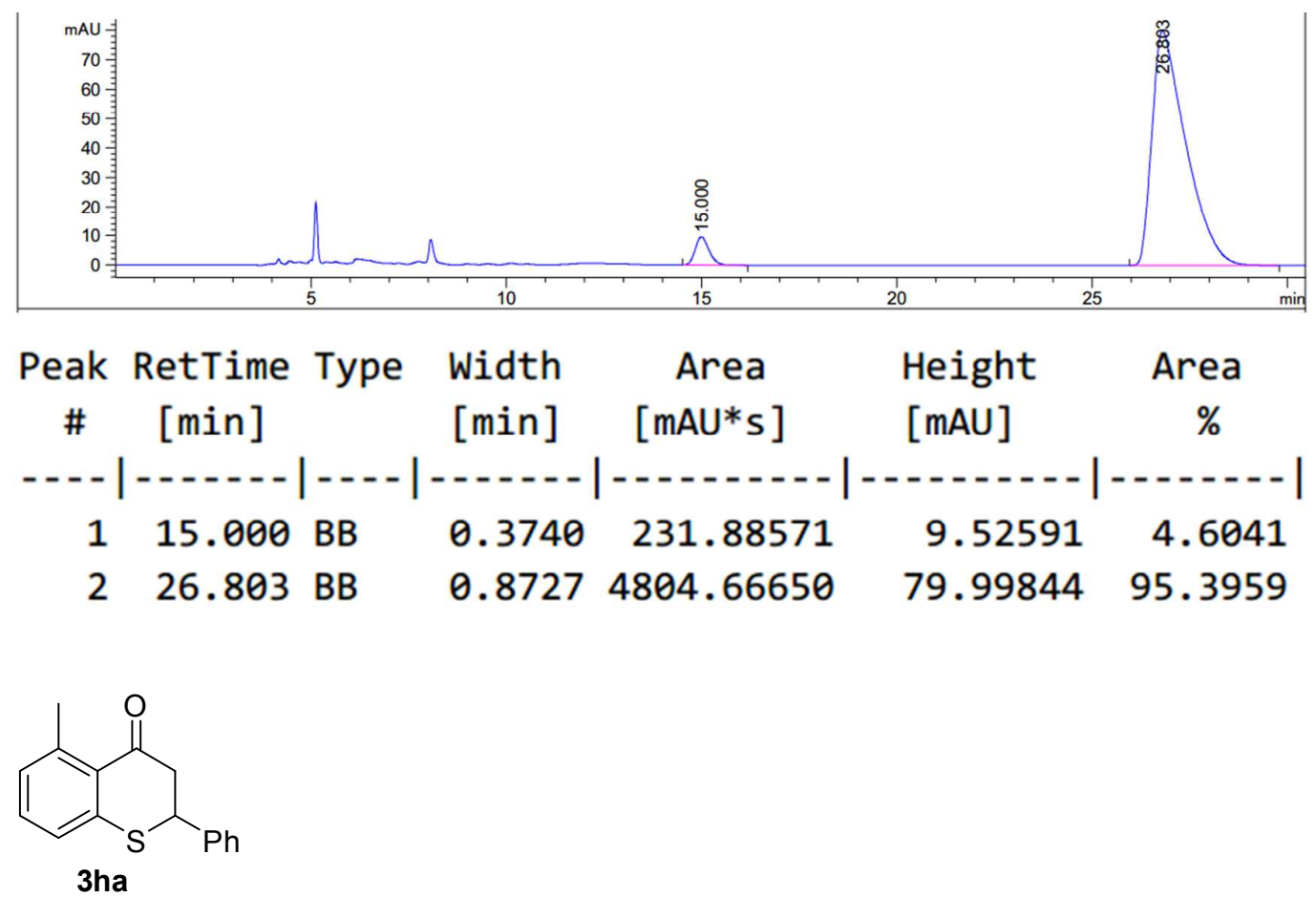

HPLC trace for racemic-3ha

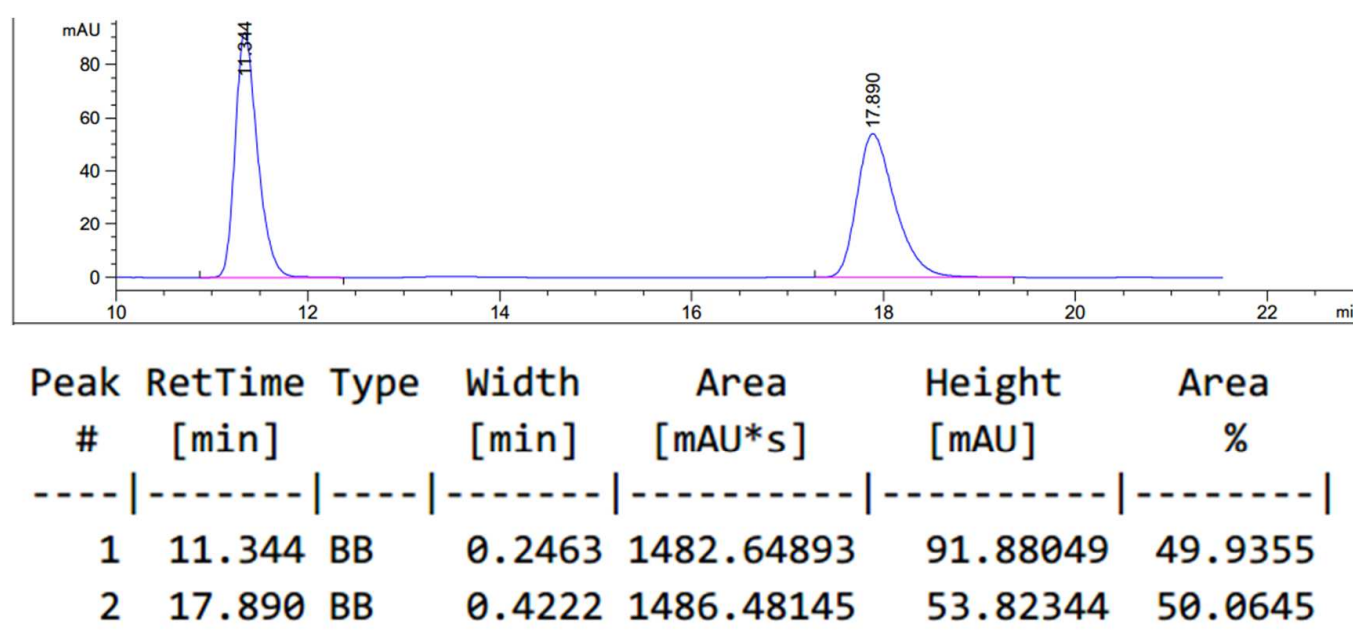

HPLC trace for enantienriched-3ha $(\mathrm{ee}=96 \%)$ 

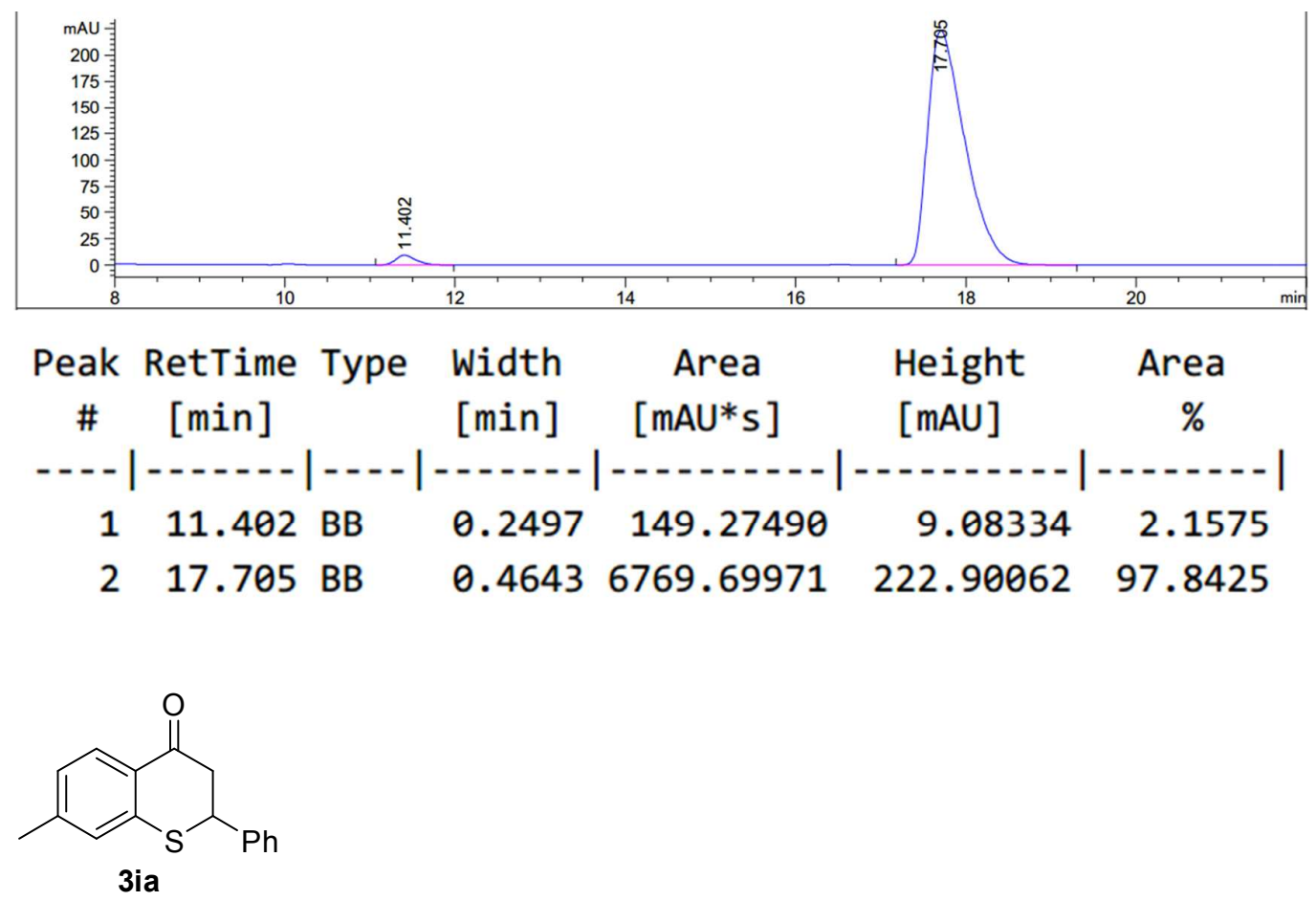

HPLC trace for racemic-3ia
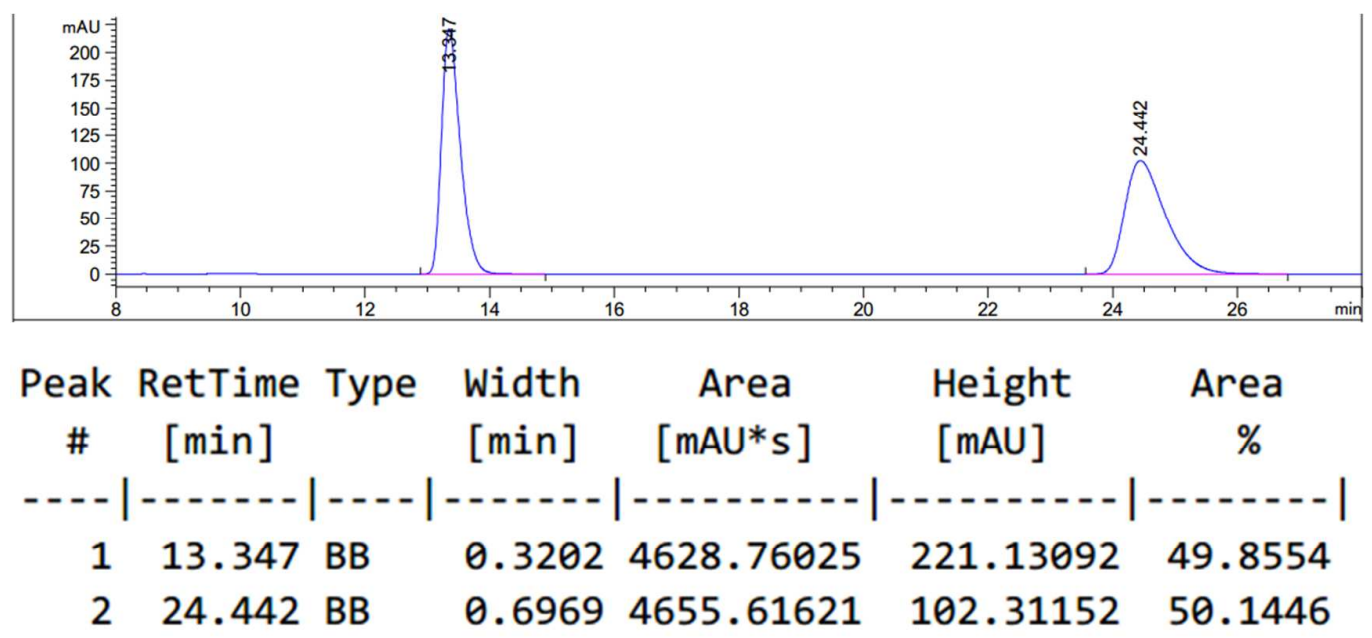

HPLC trace for enantienriched-3ia (ee $=94 \%)$

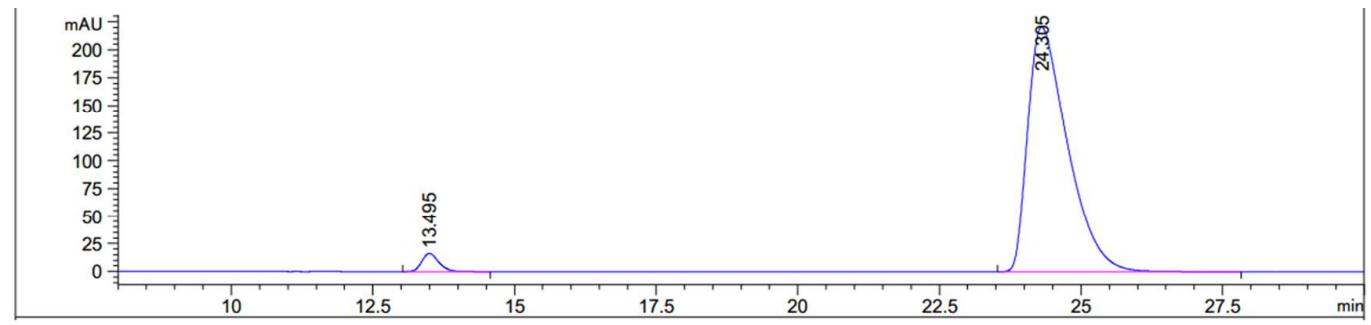



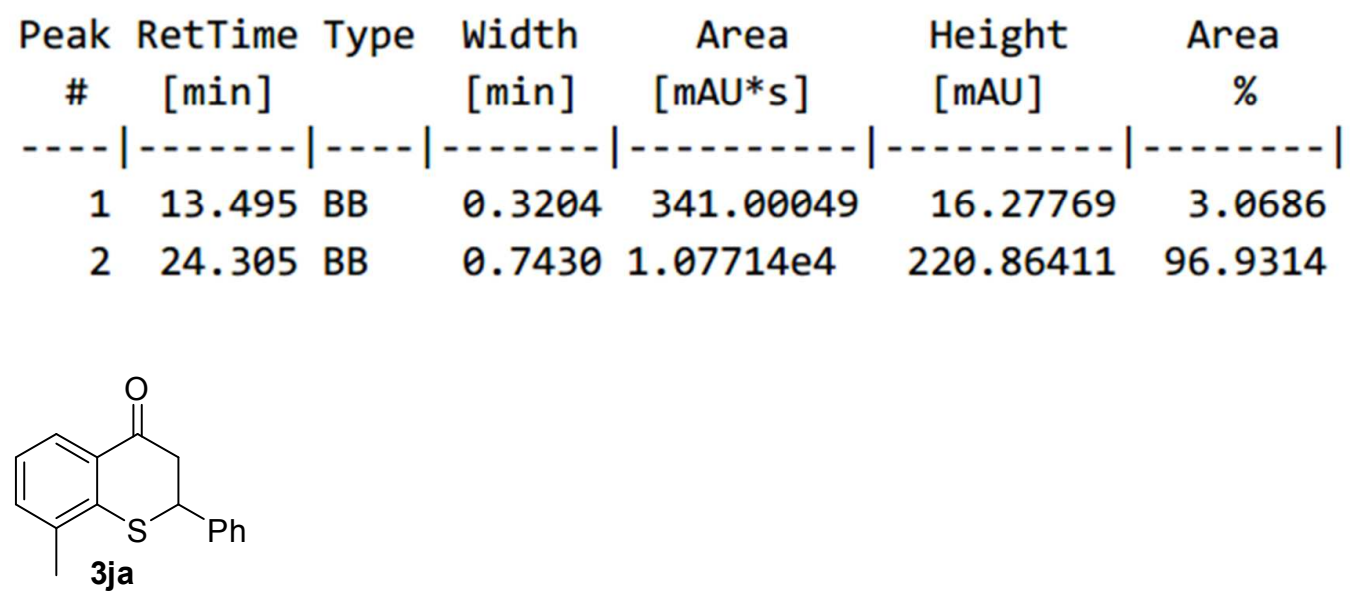

HPLC trace for racemic-3ja

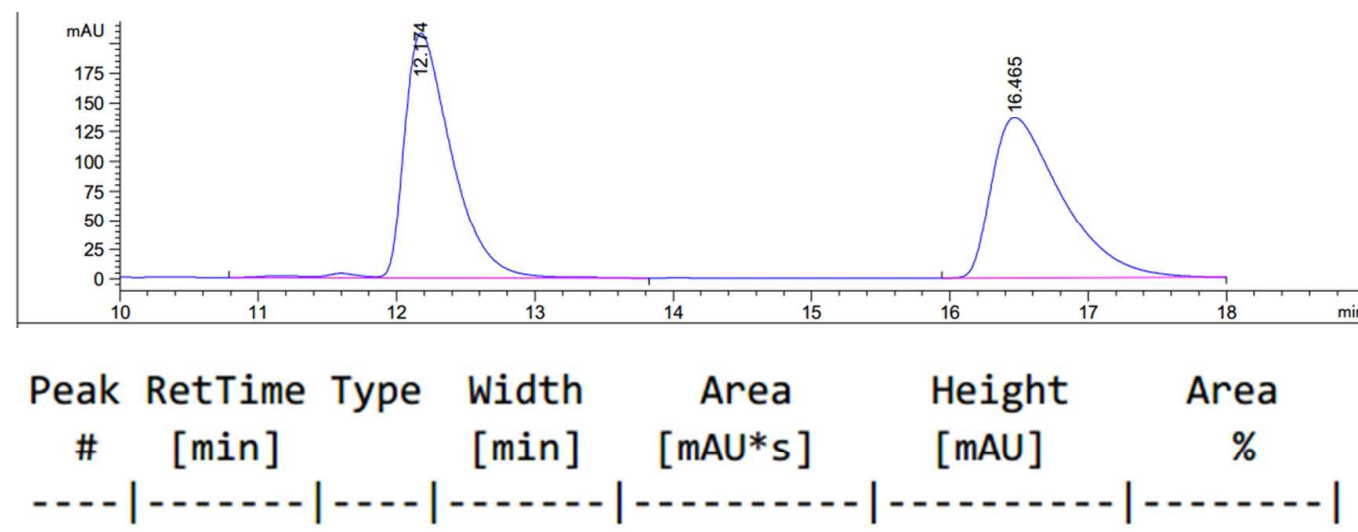

$\begin{array}{lllllll}1 & 12.174 & \text { VB R } & 0.3586 & 4984.15967 & 207.93559 & 50.8570\end{array}$

$\begin{array}{lllllll}2 & 16.465 & \text { BBA } & 0.5349 & 4816.18799 & 136.06734 & 49.1430\end{array}$

HPLC trace for enantienriched-3ja (ee $=89 \%)$

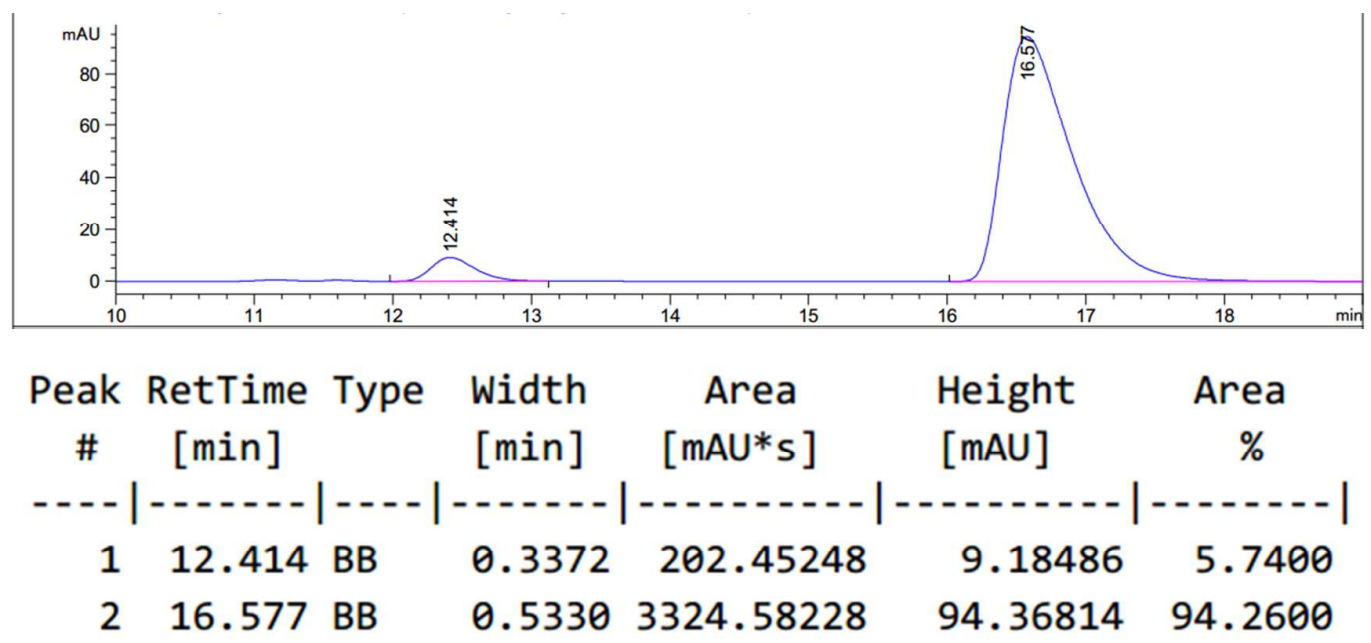




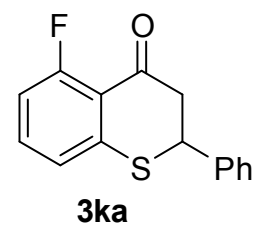

HPLC trace for racemic-3ka

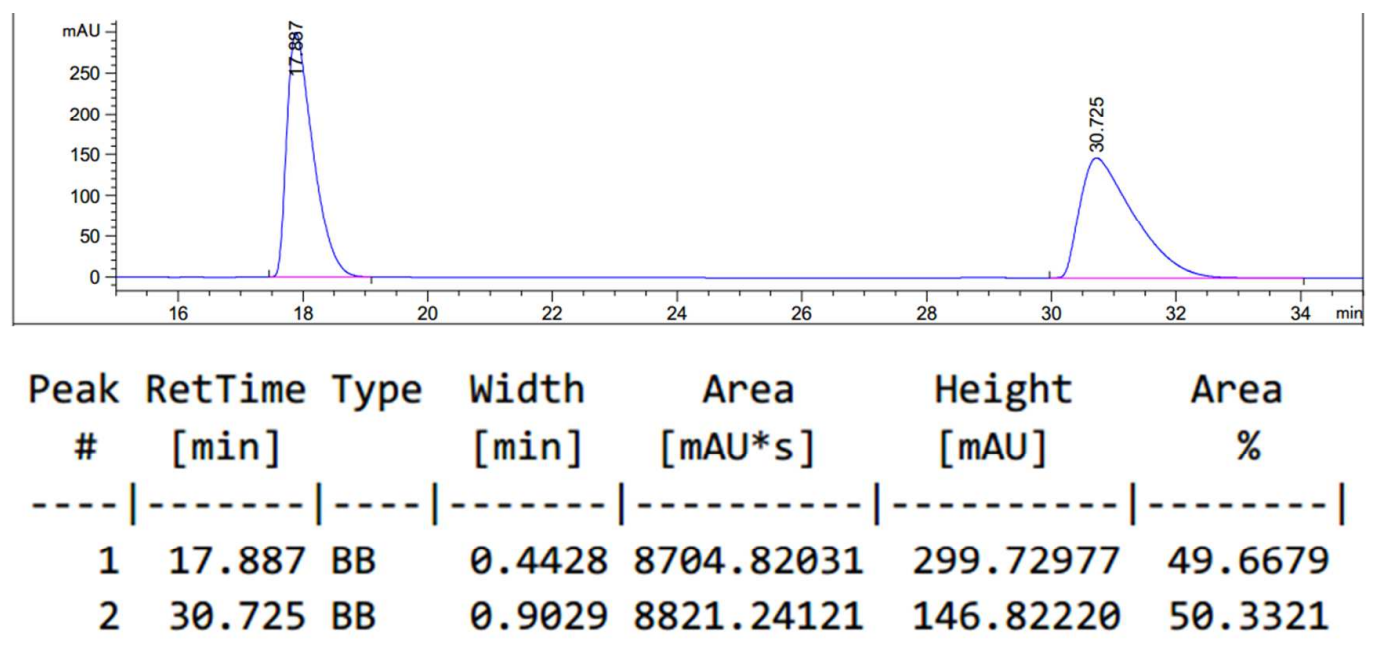

HPLC trace for enantienriched-3ka $(\mathrm{ee}=95 \%)$

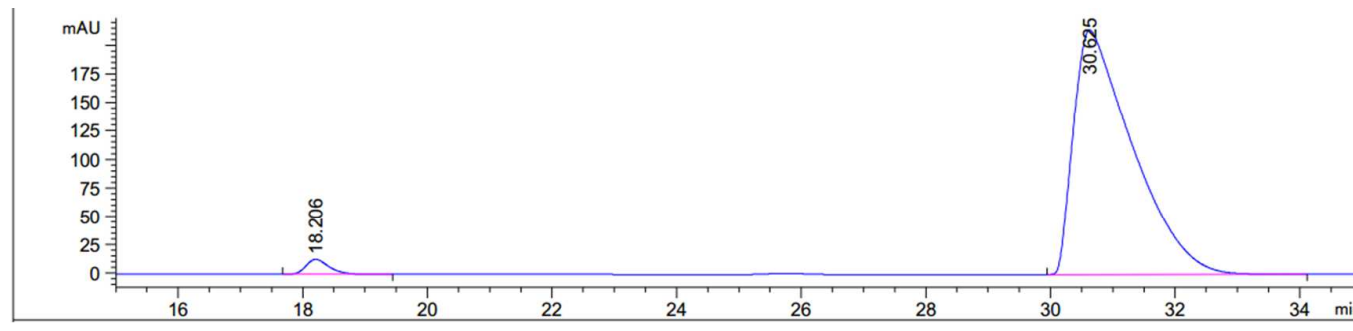

\begin{tabular}{|c|c|c|c|c|c|c|}
\hline $\begin{array}{c}\text { Peak } \\
\text { \# }\end{array}$ & $\begin{array}{c}\text { RetTime } \\
\text { [min] }\end{array}$ & Type & $\begin{array}{l}\text { Width } \\
\text { [min] }\end{array}$ & $\begin{array}{c}\text { Area } \\
{[\mathrm{mAU} * \mathrm{~s}]}\end{array}$ & $\begin{array}{l}\text { Height } \\
{[\mathrm{mAU}]}\end{array}$ & $\begin{array}{c}\text { Area } \\
\%\end{array}$ \\
\hline & & & & 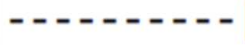 & & 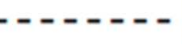 \\
\hline 1 & 18.206 & BB & 0.3883 & 332.27991 & 12.99751 & 2.3001 \\
\hline 2 & 30.625 & BB & .9597 & $1.41143 \mathrm{e} 4$ & 214.49376 & 97.6999 \\
\hline
\end{tabular}<smiles>O=C1CC(c2ccccc2)Sc2cc(F)ccc21</smiles>

HPLC trace for racemic-3la 

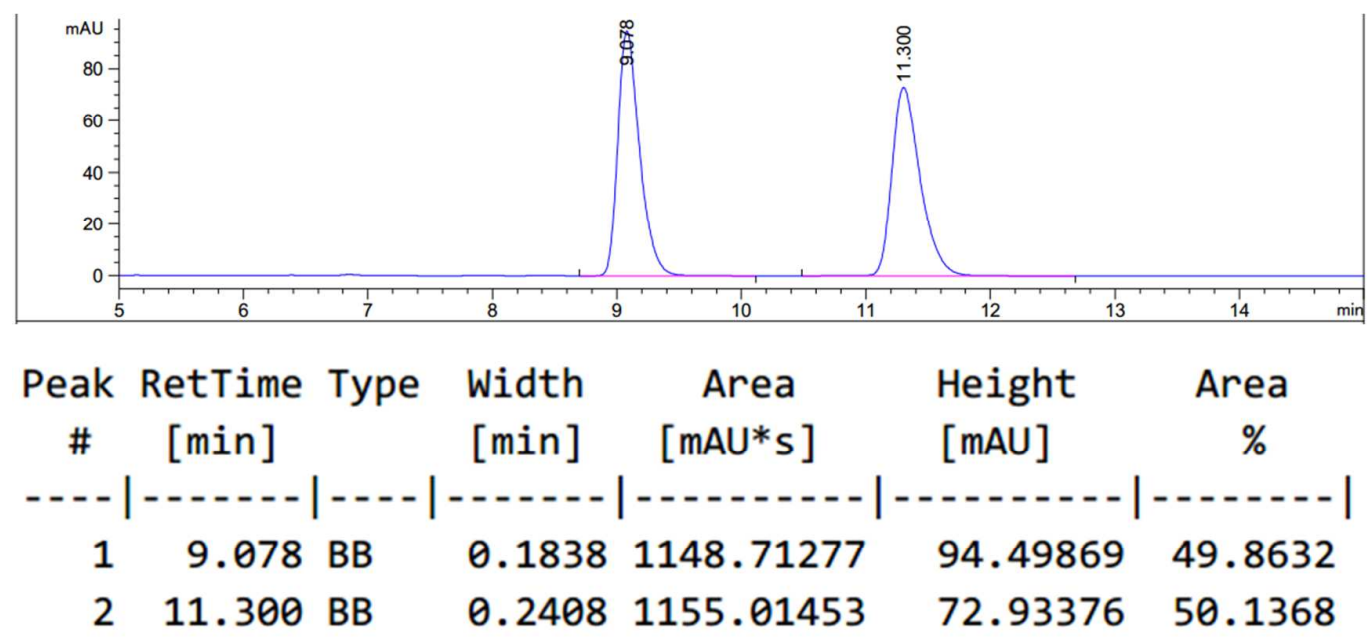

HPLC trace for enantienriched-3la (ee $=94 \%)$
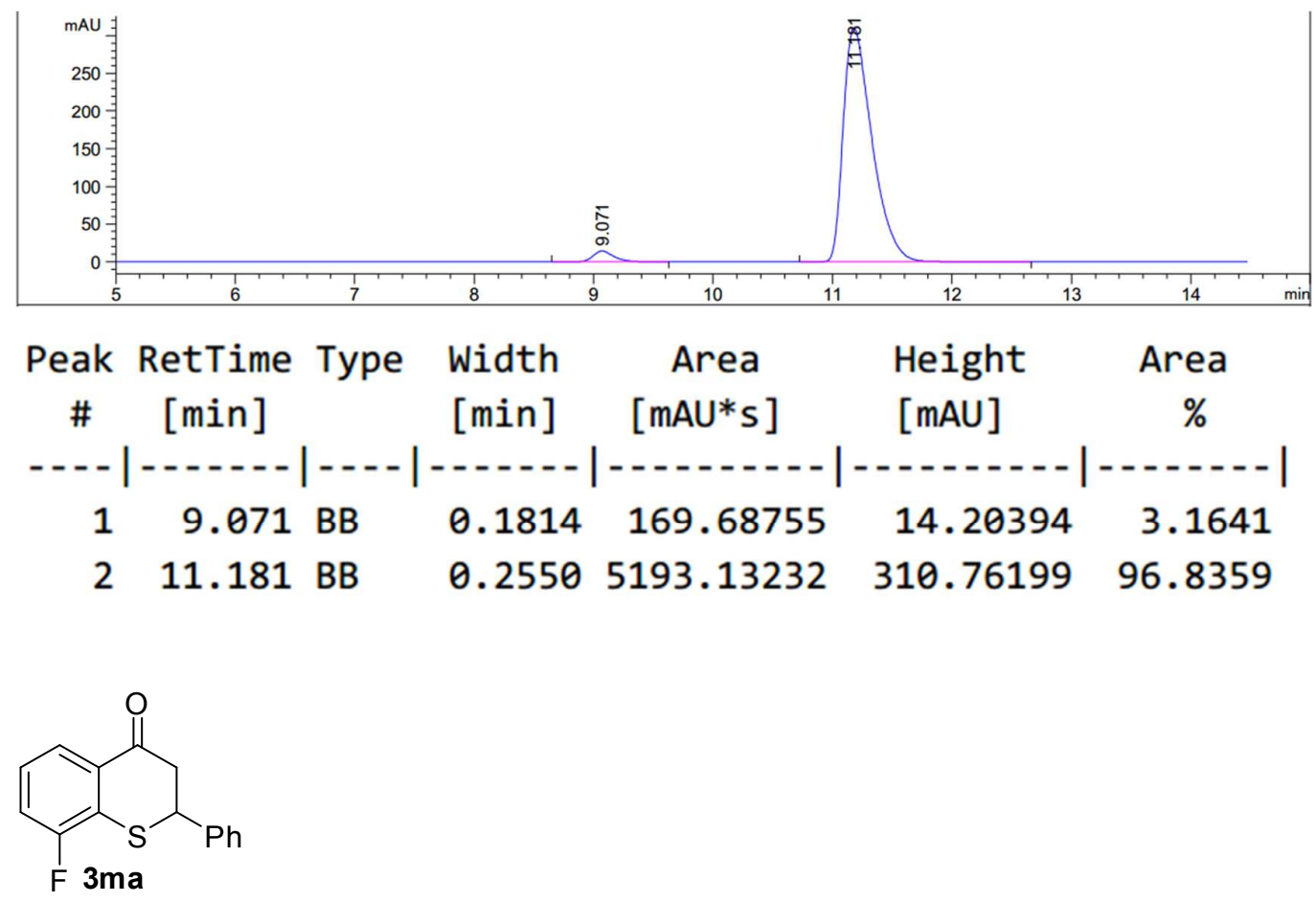

HPLC trace for racemic-3ma

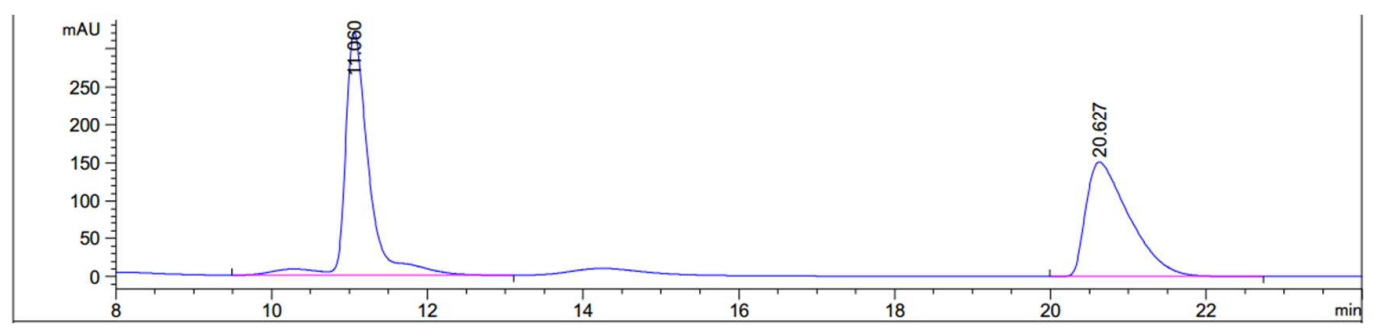




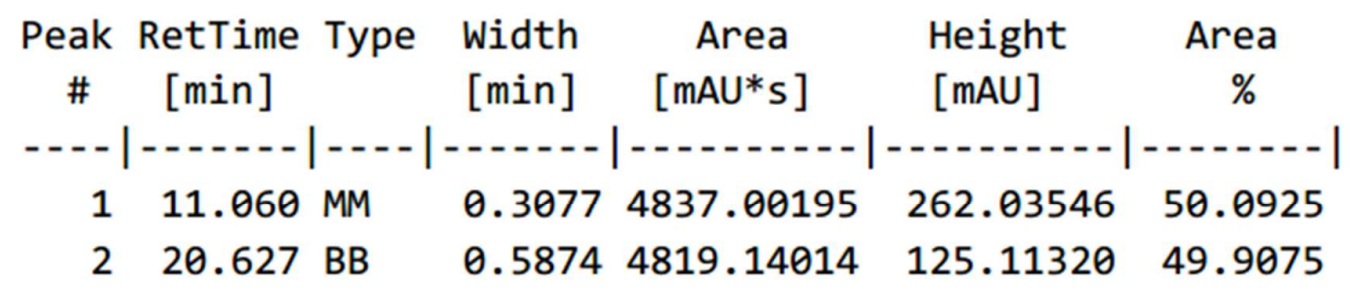

HPLC trace for enantienriched-3ma $(\mathrm{ee}=83 \%)$

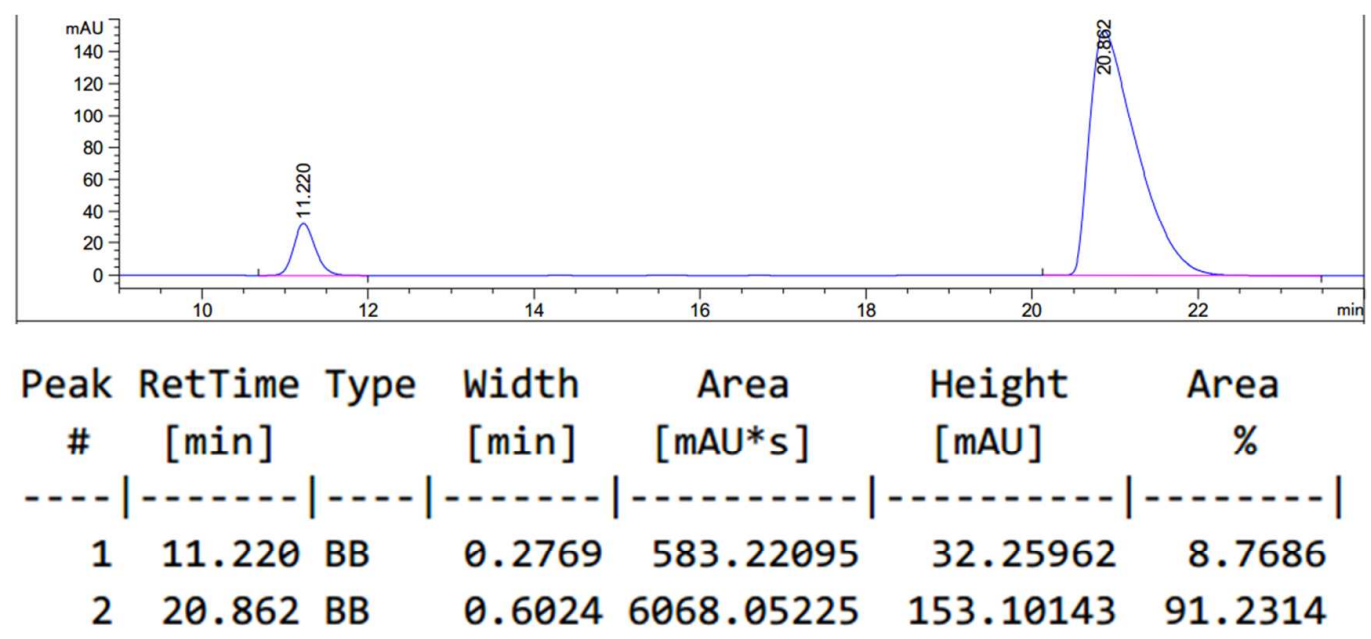<smiles>Cc1cc2c(cc1C)C(=O)CC(c1ccccc1)S2</smiles>

HPLC trace for racemic-3na

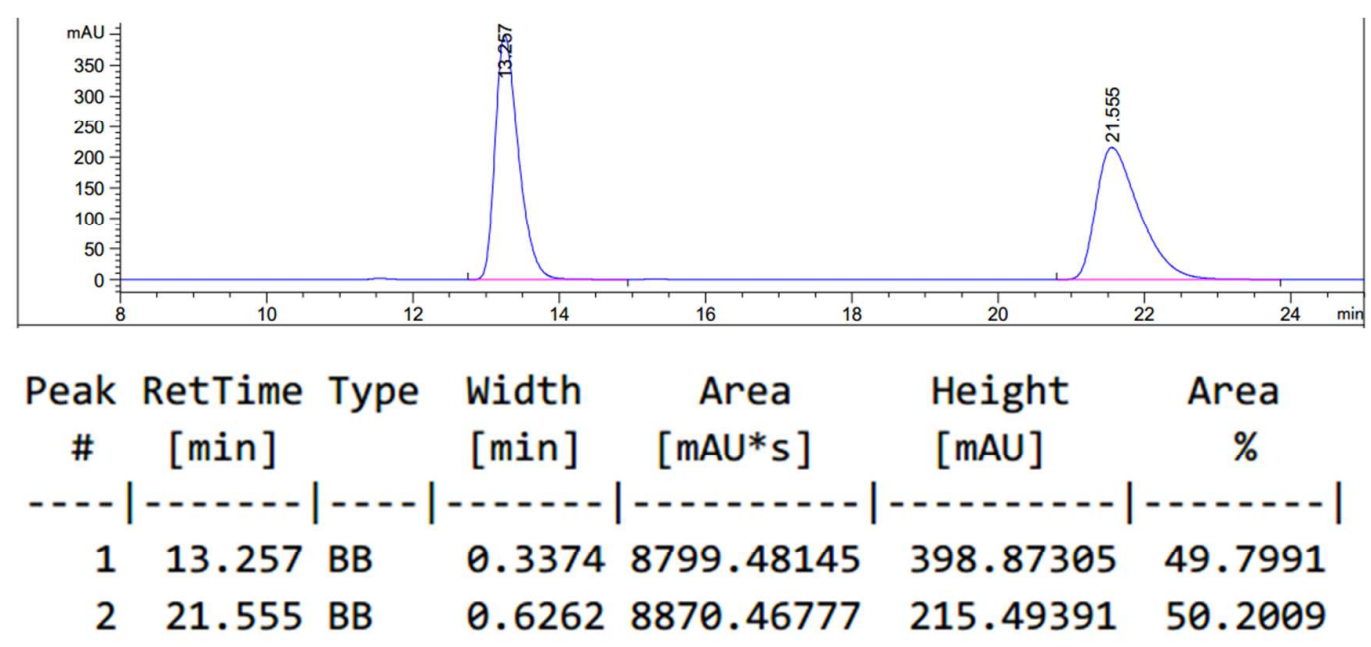

HPLC trace for enantienriched-3na $(e e=95 \%)$ 


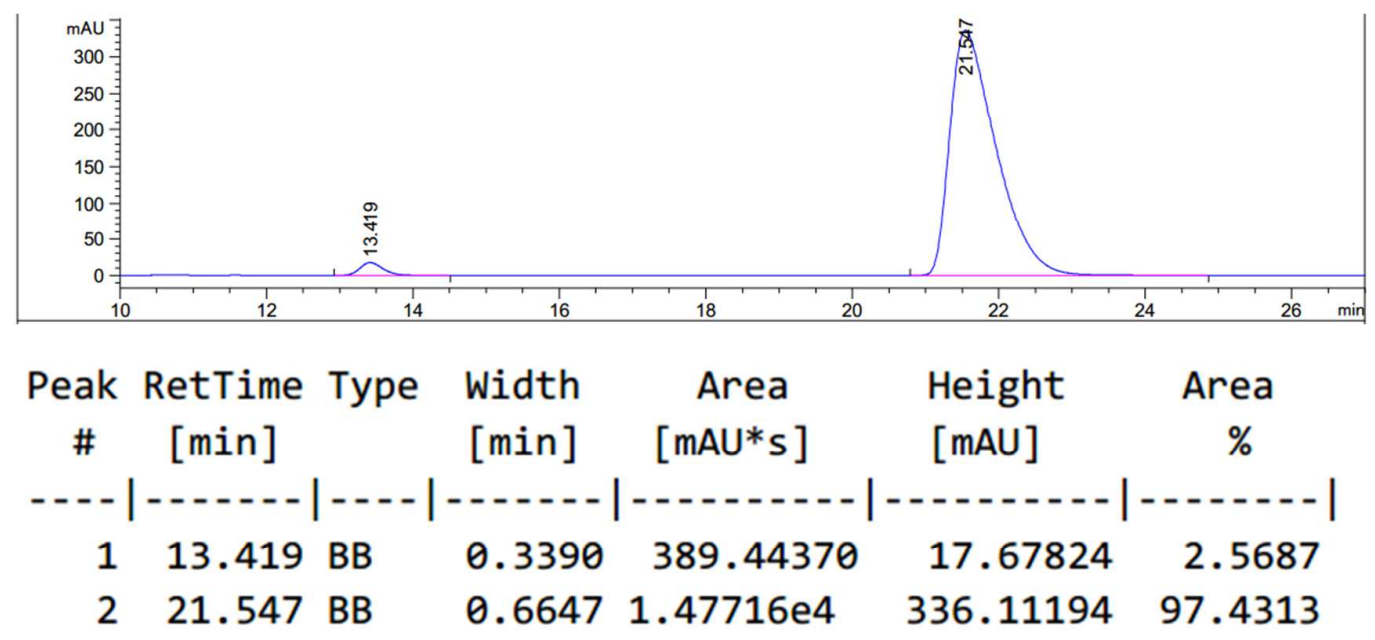<smiles>O=C1CC(c2ccccc2)Sc2cc3ccccc3cc21</smiles>

HPLC trace for racemic-3oa

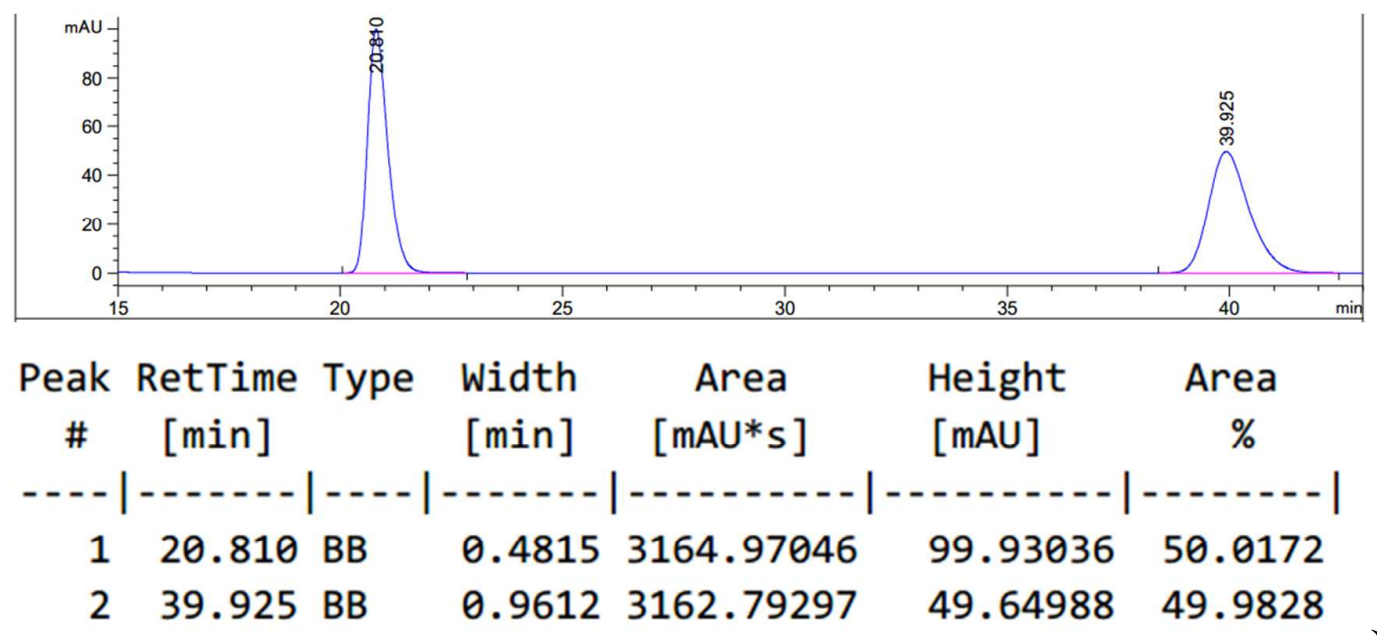

HPLC trace for enantienriched-3oa (ee $=90 \%)$

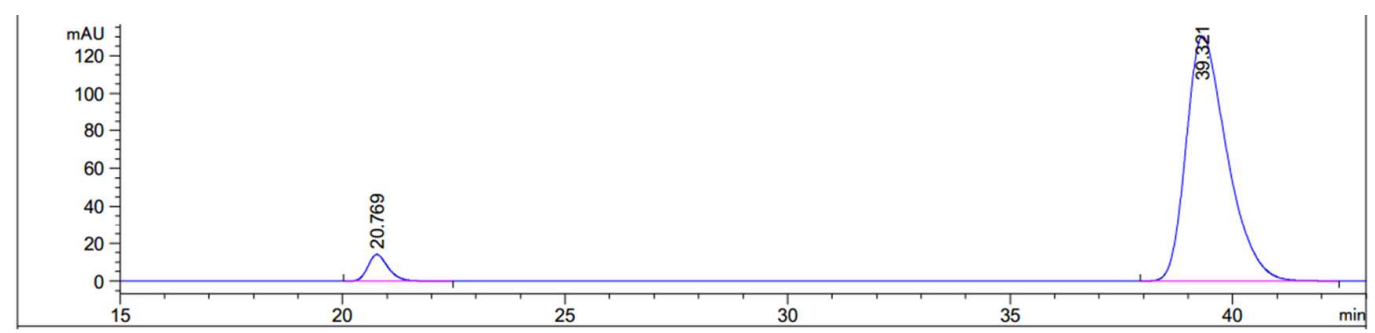




\begin{tabular}{|c|c|c|c|c|c|c|}
\hline $\begin{array}{c}\text { Peak } \\
\#\end{array}$ & $\begin{array}{c}\text { RetTime } \\
\text { [min] }\end{array}$ & Type & $\begin{array}{l}\text { Width } \\
\text { [min] }\end{array}$ & $\begin{array}{c}\text { Area } \\
{[\mathrm{mAU} * \mathrm{~s}]}\end{array}$ & $\begin{array}{l}\text { Height } \\
{[\mathrm{mAU}]}\end{array}$ & $\begin{array}{c}\text { Area } \\
\%\end{array}$ \\
\hline & & & & - & & - \\
\hline 1 & 20.769 & BB & 0.4776 & 439.48825 & 14.02638 & 5.0215 \\
\hline 2 & 39.321 & BB & .9712 & 8312.70801 & 130.14813 & 94.9785 \\
\hline
\end{tabular}

